\title{
Composite Fading Channel Modeling and Information Capacity of Distributed Antenna Architectures in Cellular Networks
}

\author{
by \\ Saad Al-Ahmadi, B.Sc., M.Sc. \\ A thesis submitted to \\ the Faculty of Graduate Studies and Research \\ in partial fulfillment of \\ the requirements for the degree of \\ Doctor of Philosophy
}

The Ottawa-Carleton Institute for

Electrical and Computer Engineering (OCIECE)

\author{
Department of Systems and Computer Engineering \\ Carleton University \\ Ottawa, Ontario, Canada
}

July 2010

Copyright (C)

2010 - Saad Al-Ahmadi 


$\begin{array}{ll}\begin{array}{l}\text { Library and Archives } \\ \text { Canada }\end{array} & \begin{array}{l}\text { Bibliothèque et } \\ \text { Archives Canada }\end{array} \\ \begin{array}{l}\text { Published Heritage } \\ \text { Branch }\end{array} & \begin{array}{l}\text { Direction du } \\ \text { Patrimoine de l'édition }\end{array} \\ \begin{array}{l}\text { 395 Wellington Street } \\ \text { Ottawa ON K1A ON4 } \\ \text { Canada }\end{array} & \begin{array}{l}395, \text { rue Wellington } \\ \text { Ottawa ON K1A ON4 } \\ \text { Canada }\end{array}\end{array}$

Your file Votre référence

ISBN: 978-0-494-70539-1

Our file Notre référence

ISBN: 978-0-494-70539-1

NOTICE:

The author has granted a nonexclusive license allowing Library and Archives Canada to reproduce, publish, archive, preserve, conserve, communicate to the public by telecommunication or on the Internet, loan, distribute and sell theses worldwide, for commercial or noncommercial purposes, in microform, paper, electronic and/or any other formats.

The author retains copyright ownership and moral rights in this thesis. Neither the thesis nor substantial extracts from it may be printed or otherwise reproduced without the author's permission.
AVIS:

L'auteur a accordé une licence non exclusive permettant à la Bibliothèque et Archives Canada de reproduire, publier, archiver, sauvegarder, conserver, transmettre au public par télécommunication ou par l'Internet, prêter, distribuer et vendre des thèses partout dans le monde, à des fins commerciales ou autres, sur support microforme, papier, électronique et/ou autres formats.

L'auteur conserve la propriété du droit d'auteur et des droits moraux qui protège cette thèse. $\mathrm{Ni}$ la thèse ni des extraits substantiels de celle-ci ne doivent être imprimés ou autrement reproduits sans son autorisation.
In compliance with the Canadian Privacy Act some supporting forms may have been removed from this thesis.

While these forms may be included in the document page count, their removal does not represent any loss of content from the thesis.
Conformément à la loi canadienne sur la protection de la vie privée, quelques formulaires secondaires ont été enlevés de cette thèse.

Bien que ces formulaires aient inclus dans la pagination, il n'y aura aucun contenu manquant. 


\section{Abstract}

The introduction of new schemes that are based on the communication among distributed ports or nodes, has motivated the use of composite fading models due to the fact that the distributed ports/nodes experience different multipath fading and shadowing statistics, which will subsequently determine the required statistics for the performance analysis of different trans-receivers. However, most of the existing work on the performance of distributed antenna systems (DASs) in cellular networks rely on lognormal shadowing models, which do not lead to closed-form expressions for the composite fading statistics and for subsequent performance metrics. In this thesis, the more tractable generalized- $K$ composite fading model is adopted. It is shown, using the moment matching method, that the generalized- $K$ distribution can be region-wise approximated by the familiar Gamma distribution and then the statistics for both the sum and the weighted sum of generalized- $K$ random variables are studied for both the independent and correlated scenarios.

Another line of research that was motivated by the introduction of DASs is the effect of the individual power constraints at each distributed antenna port (DAP) on the design and analysis of optimal/suboptimal transmission strategies. In this thesis, the optimality range of beamforming under the individual power constraints is derived for correlated two-input multi-output channels. Finally, the developed results on the statistics for the weighted sum of generalized- $K$ random variables are utilized to study the performance of DASs in both single-cell and multi-cell environments to reveal the gains of cellular DASs and to develop insights into their design. 


\section{Acknowledgement}

I would like to express my gratitude to my supervisor Dr. Halim Yanikomeroglu for his guidance during this thesis. His valuable feedback and enthusiasm for research made my doctoral study a very enjoyable and rich experience. I would also like to thank him for providing me with an ideal research environment.

I would like to thank all my colleagues in Dr. Yanikomeroglu's research group for the enjoyable research environment that we had. The residents of Lab MC 4038 have made that lab a great place for fruitful work.

I thank my parents and my brothers for their constant support and encouragement throughout my life and the course of this Ph.D. degree. Finally, I am thankful to my wife for her continual help and patience. 


\section{Contents}

$\begin{array}{lll}\text { Abstract } & \text { ii }\end{array}$

Acknowledgement iii

Contents iv

List of Figures

List of Tables $\quad$ ix

Nomenclature $\quad x$

1 Introduction 1

1.1 Cellular Systems . . . . . . . . . . . . . . . . . . . 1

1.2 Distributed Antenna Systems . . . . . . . . . . . . . 2

1.3 Objectives and Contributions $\ldots \ldots \ldots \ldots$

2 Background 6

2.1 Early Evolution of DASs . . . . . . . . . . . . . . 6

2.2 Radio over Fiber $(\mathrm{RoF}) \ldots \ldots \ldots \ldots$

3 The Generalized- $K$ Composite Fading Model and the Approximate Gamma Model $\quad 11$

3.1 Wireless Channel Modeling . . . . . . . . . . . . . . . . . 11

3.1 .1 Small-scale Fading . . . . . . . . . . . . . . . . . . 12

3.1 .2 Large-scale Fading . . . . . . . . . . . . . . . . . . 14

3.1 .3 Path Loss . . . . . . . . . . . . . . . . . . . . . 15

3.2 Composite Fading Channel Modeling . . . . . . . . . . . . . 16

3.2.1 Lognormal-based Models . . . . . . . . . . . . . . . . . 17

3.2.2 Gamma-based Models . . . . . . . . . . . . . . . . . 17

3.3 The Approximate Gamma Model . . . . . . . . . . . . . . . . 20

3.3.1 Higher-order Moment Matching . . . . . . . . . . . . . 21

3.3.2 Moment Matching Method with Adjustment . . . . . . . . . 26

3.4 Conclusions . . . . . . . . . . . . . . . . . . 30 
4 On the Statistics of the Sum of Generalized- $K$ RVs 32

4.1 Related Work . . . . . . . . . . . . . . . . . . . 32

4.2 The Approximate Gamma Distribution for the Sum of Independent Generalized-K RVs . . . . . . . . . . . . . . . . . 33

4.3 The Approximate Generalized- $K$ PDF for the PDF of the Sum of Independent Generalized- $K$ RVs . . . . . . . . . . . . . . 37

4.3.1 Case of i.i.d. Generalized- $K$ RVs . . . . . . . . . . . 37

4.3.2 Case of i.n.d. Generalized-K RVs . . . . . . . . . . . . 43

4.4 The Approximate Distribution of the Weighted Sum of Independent Generalized-K RVs . . . . . . . . . . . . . . . . . . 45

4.5 On the Statistics of the Sum of Correlated Generalized-K RVs . . . 47

4.5.1 The AF for the Sum of Identically Distributed Correlated Generalized$K$ RVs . . . . . . . . . . . . . . . . 49

4.5.2 The AF for the Sum of Non-identically Distributed Correlated Generalized- $K$ RVs . . . . . . . . . . . . . . . 52

4.6 Conclusions . . . . . . . . . . . . . . . . . . 55

5 On the Capacity of MIMO Channels with Common and Individual Power Constraint $\quad 57$

5.1 Related Work . . . . . . . . . . . . . . . . . . . . 58

5.2 Capacity of MIMO Channels with a Common Power Constraint . . . 60

5.2.1 Deterministic Channel Matrix . . . . . . . . . . . . 60

5.2 .2 Fading Channel Matrix . . . . . . . . . . . . . . 63

5.2.3 TIMO Channels with a Common Power Constraint . . . . . . 64

5.3 Capacity of MIMO Channels with Individual Power Constraints . . . 66

5.3.1 TIMO Channels with Individual Power Constraints . . . . . 66

5.4 Conclusions . . . . . . . . . . . . . . . . . 70

6 On the Capacity of Distributed Antenna Systems in Cellular Networks

6.1 Related Literature . . . . . . . . . . . . . . . . . . . 73

6.2 Centralized DAS Architectures . . . . . . . . . . . . . . . . 73

6.3 The Performance of DAS in a Single-cell Scenario . . . . . . . . . . 75

6.3.1 The Signal and Channel Model . . . . . . . . . . . . . 75

6.3.2 The Ergodic Capacity and the Information Outage Capacity of a Single-user DAS Over Independent Generalized- $K$ Channels 76

6.4 The Performance of DASs in Multi-cell Environments . . . . . . . . . 82

6.4.1 Extension to the BSs Cooperative Schemes . . . . . . . . . 85

6.5 Conclusions . . . . . . . . . . . . . . . . 86

7 Conclusions and Future Research $\quad \mathbf{8 8}$

7.1 Summary and Conclusions . . . . . . . . . . . . . . . 88

7.2 Future Research Directions . . . . . . . . . . . . . . . . . 90

$\begin{array}{ll}\text { Appendices } & 92\end{array}$ 
A The $H$-function Distribution Family $\quad 93$

B Derivations for Chapter $4 \quad 95$

B.1 Derivation of the Maximum Possible Negative Correlation Coefficient 95

B.2 Derivation of $(4.20) \ldots \ldots \ldots \ldots \ldots \ldots$

B.3 Proof of Proposition $4.2 \ldots \ldots \ldots \ldots$

$\begin{array}{lr}\text { C Derivations for Chapter } 5 & 99\end{array}$

$\begin{array}{ll}\text { D Derivations for Chapter } 6 & 104\end{array}$

E Papers Published, Submitted, and in Preparation 105

$\begin{array}{ll}\text { References } & 107\end{array}$ 


\section{List of Figures}

2.1 A basic CDMA DAS. . . . . . . . . . . . . . . 7

2.2 A typical DAS with six DAPs per cell. . . . . . . . . . 8

3.1 The Gamma distribution plots for different values of the shape param-

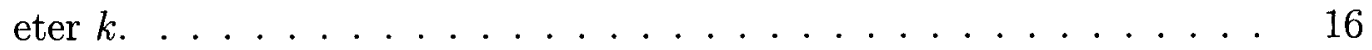

3.2 The generalized- $K$ distribution plots for different values of $m_{m}$ and $m_{s} .19$

3.3 The $\log -\log$ CDF plots of the generalized- $K$ and the approximating Gamma RVs for $m_{m}=2.5$ and $m_{s}=2.5$ using the moment matching method. . . . . . . . . . . . . . . .

3.4 The log-log CDF plots of the generalized- $K$ and the approximating Gamma RVs for $m_{m}=7$ and $m_{s}=4$ using the moment matching method.

3.5 The log-log CDF plots of the generalized- $K$ and the approximating Gamma RVs for $m_{m}=10$ and $m_{s}=10$ using the moment matching method. . . . . . . . . . . . . . .

3.6 The CDF plots of the generalized- $K$ and the approximating Gamma RVs for $m_{m}=2$ and $m_{s}=2$ using the first-two moment matching. . .

3.7 The plot of the adjustment factor that minimizes the absolute value of the difference between the approximated generalized- $K$ and the approximating Gamma distributions over the whole CDF. . . . . . . .

3.8 The plot of the adjustment factor that minimizes the absolute value of the difference between the generalized- $K$ and the approximating Gamma distributions in the lower tail of the CDF $(<0.1) \ldots \ldots$.

3.9 The ergodic capacity plot of a Rayleigh fading channel with and without shadowing. . . . . . . . . . . . .

4.1 The log-log CDF plots for the sum of generalized- $K$ RVs and the approximating Gamma RV for $m_{m}=2, m_{s}=4\left(\sigma_{s}=4.2 \mathrm{~dB}\right), \epsilon=0.2$, and different values of $N$.

4.2 The log-log (to base 10) CDF plots for the lower tail of the sum of generalized- $K$ RVs and the approximating generalized- $K$ RV for different values of $N$ with $m_{m}=m_{s}=2 . \ldots \ldots \ldots \ldots$

4.3 The $\log -\log$ (to base 10) CDF plots for the lower tail of the sum of generalized- $K$ RVs and the approximating generalized- $K$ RV for different values of $N$ with $m_{m}=m_{s}=4 \ldots \ldots \ldots \ldots$ 
4.4 The log-log (to base 10) CDF plots for the upper tail of the sum of generalized- $K$ RVs and the approximating generalized- $K$ RV for different values of $N$ with $m_{m}=m_{s}=4 \ldots \ldots \ldots \ldots$

4.5 The $\log -\log$ (to base 10) CDF plots for the sum of generalized- $K$ RVs and the approximating generalized- $K$ RV for different values of $N$ with $m_{m}=3$ and $m_{s}=6$.

4.6 The log-log (to base 10) CDF plot for the sum of two generalized- $K$ RVs and the approximating generalized- $K$ RV for $N=2$ with $m_{m, 1}=$ $2, m_{s, 1}=4$, and $m_{m, 2}=3, m_{s, 2}=1 \ldots \ldots \ldots \ldots$

4.7 The log-log (to base 10) CDF plots for the weighted sum of generalized$K$ RVs and the approximating generalized- $K$ RV for different values of $N$ with $m_{m}=m_{s}=2$ and $c_{1}=1, c_{2}=0.75, c_{3}=0.5$, and $c_{4}=0.5$.

4.8 The $\log -\log$ (to base 10) CDF plots for the weighted sum of generalized$K$ RVs and the approximating generalized- $K \mathrm{RV}$ for different values of $N$ with $m_{m}=m_{s}=4$, and $c_{1}=1, c_{2}=0.75, c_{3}=0.5$, and $c_{4}=0.5$.

4.9 The plot for the correlation coefficient between two i.d. generalized- $K$ RVs as a function of their multipath and shadowing parameters. . .

4.10 The plot for the ergodic capacity loss versus the AF and the SNR. .

5.1 A MIMO channel with $L$ transmit and $M$ receive antennas. . . . . . .

5.2 The plots of the MIMO capacity with common and individual power constraints.

5.3 The plots of the MIMO capacity with individual power constraints for different transmission schemes. . . . . . . . . . . . . . .

6.1 A multicell DAS with six DAPs per cell. . . . . . . . . . . . . .

6.2 The plot of the outage probability versus the target rate for different numbers of cooperating DAPs at $P / \sigma^{2}=10 \mathrm{~dB}$ where the $c_{i}^{\prime} s$ are as defined in (4.15) such that $c_{i}=\left(\frac{d_{1}}{d_{i}}\right)^{\beta}$.

6.3 The plot of the ergodic capacity for different numbers of cooperating DAPs. . . . . . . . . . . . . . . . . .

6.4 The plot of the approximate and actual information outage probability versus the target rate for different numbers of cooperating DAPs. .

6.5 The plot of the approximate and actual ergodic capacity for different numbers of cooperating DAPs. . . . . . . . . . . . . . 81

6.6 A cooperative multicell DAS with six DAPs per cell. . . . . . . . 85 


\section{List of Tables}

3.1 Expressions of the scale and the shape parameters of the approximating Gamma PDF obtained by moment matching (for $K_{1}, K_{2}, K_{-1}, K_{-2}$, refer to $(3.28)) \ldots \ldots \ldots \ldots \ldots \ldots \ldots$ 


\section{Nomenclature}

\section{Acronyms}

\begin{tabular}{ll}
\hline Acronym & Meaning \\
\hline AF & Amount of fading \\
AWGN & Additive white Gaussian noise \\
BER & Bit-error rate \\
CAS & Collocated antenna system \\
CDF & Cumulative distribution function \\
CCDF & Complementary cumulative distribution function \\
CDMA & Code division multiple access \\
CHF & Characteristic function \\
CSI & Channel state information \\
CSCG & Circularly symmetric complex Gaussian \\
CSIT & Channel state information at the transmitter \\
CSIR & Channel state information at the receiver \\
DAP & Distributed antenna port \\
DAS & Distributed antenna system \\
D-MIMO & Distributed multiple-input multiple-output \\
D-MISO & Distributed multiple-input single-output \\
D-SIMO & Distributed single-input multiple-output \\
i.i.d. & Independent and identically distributed \\
\hline
\end{tabular}


i.d.e.c. Identically distributed and equally correlated

KKT Karush-Kuhn-Tucker conditions

MGF Moment generating function

MIMO Multiple-input multiple-output

MISO Multiple-input single-output

MRC Maximal ratio combining

PDF Probability density function

PSD Positive semi-definiteness

RoF Radio over fiber

RV Random variable

SNR Signal-to-noise ratio

SINR Signal-to-interference-plus-noise ratio

SIR Signal-to-interference ratio

SIMO Single-input multiple-output

SISO Single-input single-output

SVD Singular value decomposition

TIMO Two-input multiple-output

UT User terminal 


\section{List of Symbols}

\begin{tabular}{ll}
\hline Symbol & Meaning \\
\hline \hline$A$ & Matrix $A$ \\
$A^{H}$ & The hermitian of a matrix $A$ \\
$A_{i j}$ & The $i, j$ entry of matrix $A$ \\
$C$ & Channel capacity \\
$\operatorname{det}(\mathrm{A})$ & Determinant of a matrix $A$ \\
$E_{X}$ & Expectation with respect to random variable $X$ \\
$I_{M \times M}$ & $M \times M$ identity matrix \\
$\operatorname{tr}(\mathrm{A})$ & Trace of a matrix $A$ \\
$\gamma$ & Instantaneous power \\
$\rho$ & Correlation coefficient \\
$\Gamma(\cdot)$ & Gamma function \\
${ }_{p} F_{q}$ & The generalized hypergeometric function, for integers $p$ and $q$ \\
$K_{m}-m_{m}(\cdot)$ & The modified Bessel function of the second kind and order $m_{s}-m_{m}$ \\
$W_{-\beta / 2, \alpha / 2}(\cdot)$ & The Whittaker function \\
$H_{p, q}^{m, n}$ & The (Fox) $H$-function with parameters $m, n, p$, and $q$ \\
$G_{p, q}^{m, n}$ & The Meijer function with parameters $m, n, p$, and $q$ \\
&
\end{tabular}




\section{Chapter 1}

\section{Introduction}

\subsection{Cellular Systems}

After the unprecedented success of the cellular industry in the early 1990s, there has been an enormous investment of resources, first, in improving cellular voice communication, and second, in exploring the possibility of wireless multimedia transmission. The promise of high data rate applications has fueled a large growth in both the wireless industry as well as in wireless research. Transmitting such information over any channel requires the satisfaction of a set of quality of service (QoS) requirements. Typical QoS requirements are that the channel consistently supports high data rates with a very small probability of error, and that the delay incurred by delay-sensitive traffic is minimal. In cellular systems the wireless medium is linked to a wireline network since an access to the backbone is required. The wireless medium, as compared to the wired medium, is less reliable and has a smaller capacity due to path loss, multipath fading, shadowing and interference. So, the design of the wireless part of the system that supports high data rates for limited bandwidth requires spectrally efficient schemes especially for cellular systems [1]. For the wireless part of the network, an intuitive proposal is to reduce the access distance of the user terminal (UT) to the wired part which will reduce the path loss and the severity of multipath fading and will consequently improve the information capacity and reliability of the 
wireless part. Another proposal is to introduce cooperation schemes among a set of adjacent base stations (BSs) to reduce the interference and improve capacity; such schemes are known as coordinated multi-point (CoMP) transmission and reception or network multiple-input multiple-output (MIMO) [2]-[5].

\subsection{Distributed Antenna Systems}

A Distributed Antenna System (DAS) is a network of spatially distributed antenna ports (DAPs) that are connected to a common source via a wired transport medium, usually optical fiber, to provide wireless service within a geographic area. DASs were originally introduced to simply cover the dead spots in indoor communications [6,7]; further studies have identified other potential advantages such as power saving and system capacity, and expanded their applications [8]-[15]. Later, research on the capacity of DASs has taken two main lines; the first line is to model DASs as variation of conventional MIMO systems where the antenna elements are geographically distributed. However, a DAS is different from a collocated MIMO system in the following aspects:

- In a DAS, each DAP has different channel gains since uplink/downlink signals between the user terminals and the different antenna ports experience different multipath fading, shadowing and path loss characteristics.

- In a DAS system, each DAP has its own power constraint whereas in collocated MIMO systems, a common power constraint is imposed.

- The dedicated links used to connect the DAPs to the central processing unit might be subject to delay or other constraints.

Due to these differences, channel modeling and performance analysis of DASs may not be carried out using existing tools for conventional collocated MIMO systems and 
different mathematical tools are needed [16].

The other line of research is based on modeling DASs in the context of multicell processing where the cooperating base stations are seen as a DAS [17]. The first approach, distributed MIMO systems, is applicable for scenarios where simple processing at the DAPs is assumed and the second approach is more applicable when advanced processing (comparable to a base station) at the DAPs is assumed.

\subsection{Objectives and Contributions}

The main objective of this thesis is to contribute toward appropriate channel modeling, optimal transmission schemes, and subsequently the design and performance analysis of DASs in cellular networks.

In Chapter 2, an introductory background about the early evolution of DASs and related topics of radio over fiber $(\mathrm{RoF})$ is presented where the literature that is particularly relevant to each chapter is reviewed. Since a very essential tool for performance analysis of DASs is the underlying composite fading model where the effects of both multipath fading and shadowing are incorporated, the main aim of Chapter 3 is to develop a simple yet sufficiently accurate composite fading model that bypasses the analytical and numerical difficulties associated with the existing models in literature. The moment-matching method is used to approximate the generalized- $K$ (GammaGamma) composite fading distribution by the mathematically more tractable Gamma distribution. The use of the Gamma distribution to model composite fading, with sufficient accuracy, significantly reduces further analytical manipulations and allows for an interesting interpretation of the shape factor of the approximating Gamma distribution as a composite fading parameter and a diversity gain measure.

In Chapter 4 , the statistics of the sum of generalized- $K$ random variables (RVs), which play an important role in the performance analysis of different diversity com- 
bining schemes over such channels, are studied. First, two moment- matching-based approximations for the distribution of the sum and the weighted sum of independent generalized- $K$ RVs are introduced. Second, the second-moment characterization, namely the amount of fading, for the sum and the weighted sum of correlated generalized- $K$ RVs is presented.

Since simple architectures of cellular DASs can be seen as a distributed MIMO system, the capacity of a point-to-point MIMO channel is revisited in Chapter 5 in order to investigate characterization of the optimal input covariance matrix for MIMO channels with individual power constraints using the standard Lagrangian formulation. Obtained results have shown relevant details on the structure of the optimal covariance matrix for two-input-multiple-output (TIMO) channels with a common power constraint and led to a characterization of the optimal transmission strategy for TIMO channels with individual power constraints.

In Chapter 6, the performance of centralized DAS architectures is considered for both single-cell and multi-cell scenarios. The developed results on the distribution of the weighted sum of generalized- $K \mathrm{RVs}$, in Chapter 4, are utilized to compute the ergodic capacity and the outage probability, and hence to quantify the gains due to the integration of DASs in cellular systems.

In a nutshell, the main contributions of this thesis can be summarized as follows:

- The development of an approximate Gamma model for the generalized- $K$ model using the moment-matching method and the introduction of the adjusted momentmatching method.

- The introduction of simple yet sufficiently accurate approximate distributions for the sum and the weighted sum of independent generalized- $K$ RVs and the development of expressions for the amount of fading for the sum of correlated generalized- $K$ RVs. 
- The derivation of the beamforming optimality range for TIMO channels with individual power constraints.

- The derivation of closed-form expressions for the ergodic capacity and the information outage probability of single-user centralized DAS arrangements in both single-cell and multi-cell environments. 


\section{Chapter 2}

\section{Background}

In this chapter, the early evolution of DASs is tracked and the closely related topic of communication over fiber optic links is introduced.

\subsection{Early Evolution of DASs}

Early proposals on the use of distributed antennas were for indoor communications [7] where measurements have shown that the propagation loss and the delay spread encountered in large buildings may render the use of a single central antenna ineffective. In [7], replacing the single antenna of each central station by a DAS, or simply a "leaky feeder" that winds its way through the hallways of the building was proposed as a solution and it was shown by measurements that the signal attenuations, in a large building, can be reduced by tens of decibels and that the delay spread can be limited to only tens of nanoseconds. There is no additional signal processing needed at the antenna elements except for amplifiers and down-converters. Although delay may incur naturally from the cable, delay elements are inserted between the antenna elements such that signal received from different antennas can be distinguished and the user terminal is expected to experience path diversity gain against multipath and shadow fading (using rake receivers).

Later, proposals on the use of DASs in code division multiple access (CDMA) 
systems appeared in [8] -[15] where again the distributed antennas are integrated in a way that creates deliberate multipath so that the signals received from two antennas are distinguishable by the temporal processing at the receiver as shown in Fig. 2.1.

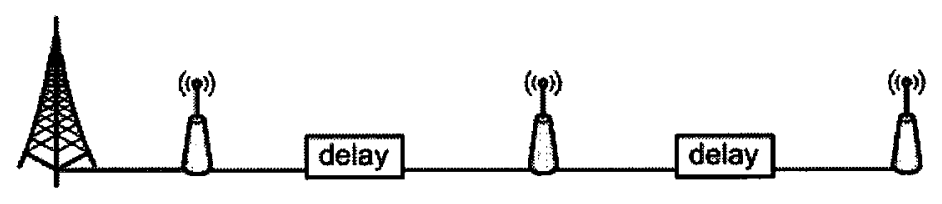

Figure 2.1: A basic CDMA DAS.

In [10], a string of three distributed nodes was used for coverage in a $300 \mathrm{ft}$ long by $100 \mathrm{ft}$ wide two-story office building. The node antennas were placed above the dropped ceiling tiles of the first floor with the dipole pointing up in order to cover both floors of the building. Each node consists only of two separate simple transmitreceive paired antenna elements where each antenna element has an amplifier and a surface acoustic wave (SAW) delay element. The SAW delay element inserts two microseconds time delay between the nodes and 8 microseconds time delay between the two radiating elements within the node. A pair of coaxial cables was used to distribute the transmitted signals to the radiating elements in the nodes. Another pair of coaxial cables was used to receive signals from all antenna elements in the nodes. All signal processing is done at the base station located within the building. It was observed that the mobile transmit power is reduced by more than $10 \mathrm{~dB}$ on average in the three-node distributed antenna system. The authors argue that this result implies that ten times more simultaneous mobile users can be contained in the DAS for a direct sequence (DS)-CDMA system whose capacity is interference limited (single-user decoders). A similar structure was considered in [12] for outdoor systems and it was found that with only four distributed antenna elements, the median and peak power levels can be reduced by $13 \mathrm{~dB}$ and $20 \mathrm{~dB}$, respectively. 


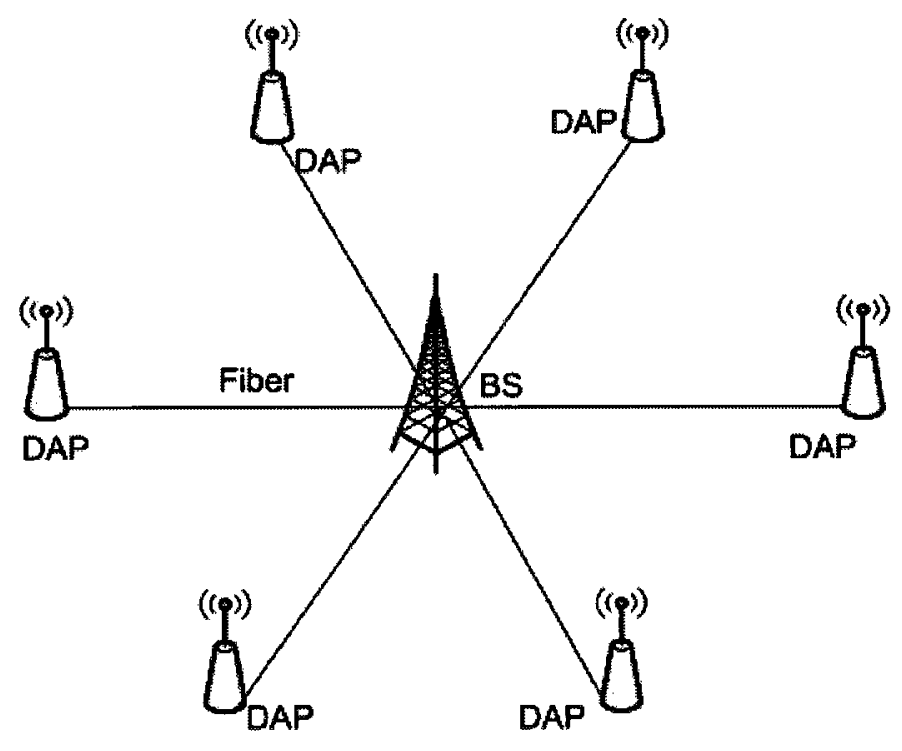

Figure 2.2: A typical DAS with six DAPs per cell.

Further evolution of DASs for outdoor cellular networks where a certain arrangement of DAPs is integrated in each cell $[18,19]$ (see Fig. 2.2) has intersected with two other emerging topics in wireless communications: radio over fiber and MIMO schemes. In this chapter, the first topic will be introduced; MIMO schemes will be discussed later in Chapter 5.

In WINNER I/II, DASs were proposed under the concept of multi-user spatial scheduling for space-division multiple access (SDMA) [20] and were introduced in Future Technologies for a Universal Radio Environment (FuTURE) in China [21]. In Long-Term-Evolution LTE-Advanced standards, DASs belong to the family of Cooperative multi-point transmission and reception (CoMP) schemes that are proposed as one of the enabling technologies to support the high data requirements. 


\section{$2.2 \quad$ Radio over Fiber (RoF)}

The use of fiber optic links in cellular systems was proposed in $[22,23]$ to connect the microcells to the main base station. This has led to the emergence of radio over fiber networks where radio frequencies are carried over optical fiber links to support various wireless applications.

Optical fiber is a dielectric medium for carrying information from one point to another in the form of light. Fiber is essentially a very thin cylindrical glass waveguide consisting of two parts: an inner core material and an outer cladding material. The core and cladding material are designed so as to keep the light signals guided inside the fiber, allowing the light signal to be transmitted for reasonably long distances with a small loss. The invention of the low-loss silica-based optical fiber in the early 1970s has led to a take-off in the use of fiber for communication. The silica-based optical fiber has three low-loss windows in the $0.8,1.3$, and $1.55 \mu \mathrm{m}$ infrared wavelength bands. The loss is around $0.25 \mathrm{~dB} / \mathrm{km}$ in the $1.55 \mu \mathrm{m}$ band, about $0.5 \mathrm{~dB} / \mathrm{km}$ in the $1.3 \mu \mathrm{m}$ band, and $2.5 \mathrm{~dB} / \mathrm{km}$ in $0.8 \mu \mathrm{m}$ [24]. The minimum loss reported for fiber optic is $0.148 \mathrm{~dB} / \mathrm{km}$ at a wavelength of $1.57 \mu \mathrm{m}$ [25] using pure-silica-core fiber to reduce the Rayleigh scattering loss which is caused by the density fluctuations of the glass and is the dominant factor in determining the transmission loss of an optical fiber. Other minor sources of loss consists of imperfection loss between core and cladding, infrared absorption loss, bending loss and hydroxyl $(\mathrm{OH})$ absorption loss [25].

Experimentally, optical signals have been sent over hundreds of kilometers without amplification. Besides its enormous bandwidth and low attenuation, fiber also offers low error rates. Communication systems using an optical fiber typically operate at a bit error rate (BER) of less than $10^{-11}[26]$. The small size and thickness of fiber allows more fiber to occupy the same physical space as copper, a property that is desirable when installing local networks in buildings. Fiber is reliable in corrosive environments, 
immune to electromagnetic interference, and does not cause interference [23].

Unlike conventional optical networks where digital signal is mainly transmitted, RoF is fundamentally an analog transmission system because it distributes the radio waveform, directly at the radio carrier frequency. Actually, the analog signal that is transmitted over the optical fiber can either be RF (radio frequency) signal, IF (intermediate frequency) signal, or baseband (BB) signal. At the optical transmitter, the $\mathrm{RF} / \mathrm{IF} / \mathrm{BB}$ signal can be imposed on the optical carrier by using direct or external modulation of the laser light. In an ideal case, the output signal from the optical link will be a copy of the input signal. The transmission of analog signals puts certain requirements on the linearity and dynamic range of the optical link [26]. 


\section{Chapter 3}

\section{The Generalized- $K$ Composite Fading Model and the Approximate Gamma Model}

As indicated in the previous chapter, modeling of composite fading channels, where the multipath fading and shadowing effects are incorporated, is essential for analyzing the performance of different communication schemes of DASs. In this chapter, first, an overview of the current models of multipath fading and shadowing is given and then the generalized- $K$ composite fading model is presented. Second, the approximation of this model by the simpler Gamma model, using the moment matching method, is explored. The limitations associated with matching higher order positive and negative moments are alleviated through the proposal of an adjustable form of the first two positive moment matching method.

\subsection{Wireless Channel Modeling}

In wireless channels, the radiated electromagnetic wave interacts in a complicated way with the medium between the transmitter and the receiver. So global deterministic characterization is not possible and can only be specific; therefore, the only way to characterize such channel is probabilistic. The incident wave interacts with surface irregularities via diffraction, scattering, reflection, and absorption, creating 
a continuum of scattered partial waves. The amplitudes and phases of these partial waves depend on the physical properties of the surface structure such as geometrical proportions and electromagnetic reflection properties. At every point in space, scattered partial waves interfere with each other and, possibly, with the direct wave, building up an irregular electromagnetic field. On the other hand, the signal power tends to decrease with distance and the existence of large scatterers such as trees, buildings, and mountains, introduces random variations of the local mean of the envelope or equivalently the local mean power. To statistically model wireless channels, it is a common practice to consider these small-scale and large-scale propagation mechanisms independently.

\subsubsection{Small-scale Fading}

Small-scale fading is due to the superposition of the received multipath signals which are due to the processes of reflection, diffraction and scattering. So, within a scale that is comparable to the carrier wavelength, the superposition of the multipath signals may add constructively (in-phase) or destructively (out-of-phase) causing the phenomenon of small-scale fading. To derive the statistical characteristics of the received wave-field that is due to the superposition of partial waves, the complex phasor of the received signal can be expressed as [27, 28]

$$
\tilde{E}=\sum_{n} E_{n} \exp (j \varphi)
$$

where $E_{n}$ is the magnitude of the $n$th path and $\varphi$ is the relative phase, which are joint random variables whose distributions are dependent on the physical properties of the propagation medium. For a mobile receiver, $E_{n}$ and $\varphi$ become stochastic processes. 
The magnitude of the complex phasor in (3.1) is defined as [27]

$$
r=\left|\sum_{n} E_{n} \exp (j \varphi)\right| .
$$

In order to derive its stationary distribution, the following assumptions are usually made [27]:

- A large number of partial waves are received with identical amplitudes.

- No correlations exist among the different partial waves.

- The magnitude and phase of each partial wave are uncorrelated.

- The relative phase of each partial wave is uniformly distributed over $[0,2 \pi]$.

Under these assumptions, it can be invoked by the central limit theorem, that the field in (3.1) is a complex Gaussian RV whose real and imaginary components are zeromean Gaussian RVs with equal variance of $1 / 2$. It is well known that the magnitude is a Rayleigh RV whose probability density function (PDF) can be expressed as [29]

$$
p(r)=\frac{r}{\sigma^{2}} \exp \left(-\frac{r^{2}}{2 \sigma^{2}}\right)
$$

If a Line-of-Sight (LOS) is present (with a non-zero mean, $A_{0}$ ), the magnitude of the received signal has a Rician distribution [30]

$$
p(r)=\frac{r}{\sigma^{2}} \exp \left[-\frac{\left(r^{2}+A_{0}^{2}\right)}{2 \sigma^{2}}\right] I_{0}\left(\frac{A_{0} r}{\sigma^{2}}\right)
$$

where $I_{0}(\cdot)$ denotes the modified Bessel function of the first kind with zero order. Another PDF that is versatile enough to include the Rayleigh PDF as a special case 
and approximate the Rician distribution ${ }^{1}$ is the Nakagami distribution[32]

$$
p(r)=\frac{2}{\Gamma\left(m_{m}\right)}\left(\frac{m_{m}}{\Omega}\right)^{m_{m}} r^{2 m_{m}-1} \exp \left(-\frac{m_{m} r^{2}}{\Omega}\right)
$$

where $\Gamma[\cdot]$ is the Gamma function defined in [33, Eqn. 8.310] as $\Gamma(z)=\int_{0}^{\infty} t^{z-1} e^{-t} d t, z \geq$ $0, \Omega=E\left(r^{2}\right)$, and $m_{m}$ is the multipath fading parameter that varies from $m_{m}=1 / 2$ for one-sided Gaussian PDF, to $m_{m}=1$ for Rayleigh PDF, and $m_{m}=\infty$ for the non-fading scenario. In [27], it was shown that the Nakagami PDF is the best approximate solution of the signal magnitude distribution of the sum of partial waves under the most general mathematical conditions. Other distributions such as Weibull distribution [34, 35], Laguerre series-based distributions [36], and the $\kappa-\mu$ distribution and the $\eta-\mu$ distribution [37] were proposed to model the signal envelope fading statistics.

\subsubsection{Large-scale Fading}

Due to the scattering caused by the general terrain, large buildings and vegetation, the local mean received power in a wireless channel varies; this phenomenon is referred to as shadowing. These variations are usually modeled by a lognormal RV ([38] and references therein)

$$
p(x)=\frac{1}{\ln 10 / 10 \sqrt{2 \pi} \sigma x} \exp \left[-\frac{\left(10 \log (x)-\mu_{s}\right)^{2}}{2 \sigma_{s}^{2}}\right],
$$

where $\mu_{s}$ is called the area mean (since averaging over shadowing effect takes place over a large area) and is determined by the propagation path loss; however, we may treat the path loss independently so that we may set $\mu_{s}=0 d B$. The standard deviation, $\sigma_{s}^{2}$ varies with the propagation environment. Typical reported values of the

\footnotetext{
${ }^{1}$ More details about the accuracy of such an approximation are given in [31].
} 
shadowing standard deviation in macrocells range from 5 to $12 \mathrm{~dB}$ and from 4 to 13 $\mathrm{dB}$ in microcells. Moreover, empirical measurements indicated that the value of $\sigma_{s}$ decreases as the scatterer's density increases ([39] and references therein). Mathematically, the lognormal model is based on invoking the central limit theorem which implies that the product of a sufficient number of independent RVs may be approximated as being lognormally distributed [38].

Another model which was adopted recently in literature to model shadowing in wireless channels is the Gamma distribution [40, 41]:

$$
p(x)=\frac{x^{k-1}}{\Gamma(k) \theta^{k}} \exp \left(-\frac{x}{\theta}\right)
$$

where $\theta$ and $k$ denote the scale and shape parameters of the Gamma distribution, respectively. The PDF plots for the Gamma distribution (with a mean of unity) for different values of the shape parameter are shown in Fig. 3.1. For small values of $k$, the PDF has skewed exponential shape and a symmetric shape for large values of $k$. In fact, the Gamma distribution converges to a Gaussian distribution with a mean of $k \theta$ and a variance of $k \theta^{2}$. The Gamma distribution has shown a good fit to measurements $[40,42]$ and is analytically more tractable than the lognormal distribution as will be seen in the next section.

\subsubsection{Path Loss}

Empirical measurements in wireless channels have shown that the average received power is dependent on the distance between the transmitter and the receiver, $d$, leading to the well-known law

$$
P(d)=\alpha\left(\frac{d}{d_{0}}\right)^{\beta}
$$




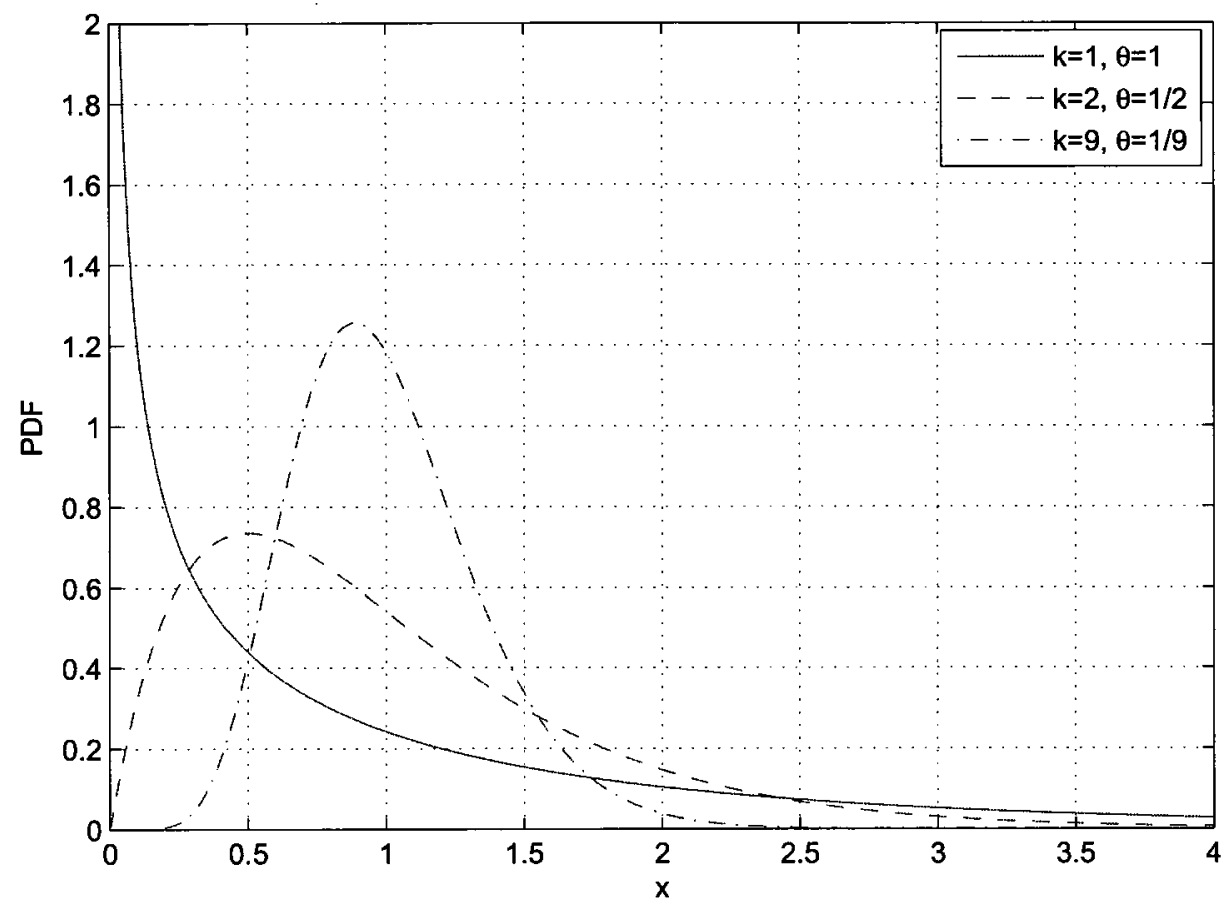

Figure 3.1: The Gamma distribution plots for different values of the shape parameter $k$.

where $d_{0}$ is a reference distance, $\alpha$ is a scale parameter that depends on the transmit wavelength, transmit and receive antenna radiation patterns, and $\beta$ is the path-loss exponent, which varies from less than 2 in guided propagation scenarios to 6 in indoor and dense urban environments.

\subsection{Composite Fading Channel Modeling}

Modeling composite fading channels, where the multipath fading and shadowing are modeled jointly, is essential for the performance analysis of DASs since the geographically distributed ports experience different multipath fading and shadowing statistics. Small-scale fading, as discussed in the previous section, is usually modeled using Rayleigh, Rician, and Nakagami distributions. The latter one is general enough to include the Rayleigh distribution as a special case and to approximate the Rician distribution. 


\subsubsection{Lognormal-based Models}

Large-scale (shadow) fading is usually modeled to have a lognormal distribution [38]. However, the lognormal-based composite fading models do not lead to closed form expressions of the received signal power distribution which hampers further analytical derivations. These models include the Rayleigh-lognormal (Suzuki) model [39] and the Nakagami-lognormal (Gamma-lognormal) model [39, 43]. Assuming that the envelope of the received signal, due to small-scale multipath fading, is modeled by the Nakagami distribution, the PDF of the received power $\gamma$, conditioned on the local mean power $\Omega$, takes the form of a Gamma distribution as

$$
p_{\gamma / \Omega}(x)=\frac{\left(\frac{m_{m}}{\Omega}\right)^{m_{m}}}{\Gamma\left(m_{m}\right)} x^{m_{m}-1} \exp \left(-\frac{m_{m} x}{\Omega}\right), \gamma \geq 0, m_{m} \geq 0.5
$$

and the Nakagami-lognormal (or Gamma-lognormal) composite fading model can be expressed as [39] as

$$
p_{\gamma}(x)=\int_{0}^{\infty} \frac{\left(\frac{m_{m}}{w}\right)^{m_{m}}}{\Gamma\left(m_{m}\right)} x^{m_{m}-1} \exp \left(-\frac{m_{m} x}{w}\right) \frac{1}{\ln 10 / 10 \sqrt{2 \pi} \sigma w} \exp \left[-\frac{\left(10 \log (w)-\mu_{s}\right)^{2}}{2 \sigma_{s}^{2}}\right] d w
$$

The expression in (3.10) reduces for $m_{m}=1$ to the Rayleigh-lognormal (exponentiallognormal) model.

\subsubsection{Gamma-based Models}

As an alternative, it has been proposed to use the more tractable Gamma density function to model the local mean power random variations due to shadowing [40,41, $44,45]$ as

$$
p_{\Omega}(y)=\frac{\left(\frac{m_{s}}{\Omega_{0}}\right)^{m_{s}}}{\Gamma\left(m_{s}\right)} y^{m_{s}-1} \exp \left(-\frac{m_{s} y}{\Omega_{0}}\right), y \geq 0, m_{s}>0,
$$

where $m_{s}$ is shadowing parameter and $\Omega_{0}$ is the average of the local mean power. 
Hence, we may write

$$
p_{\gamma}(x)=\int_{0}^{\infty} p_{\gamma / \Omega}(x) p_{\Omega}(y) d y
$$

Using (3.9) and (3.11), the PDF of the unconditional instantaneous power $\gamma$ in a Gamma-Gamma composite fading channel can be derived as [44]

$$
p_{\gamma}(x)=\frac{2 b^{m_{m}+m_{s}}}{\Gamma\left(m_{m}\right) \Gamma\left(m_{s}\right)} x^{\left(m_{m}+m_{s}\right) / 2-1} K_{m_{s}-m_{m}}(2 b \sqrt{x}) .
$$

In (3.13), $K_{m_{s}-m_{m}}(\cdot)$ is the modified Bessel function of the second kind and order $m_{s}-m_{m}$ and $b=\sqrt{\frac{m_{m} m_{s}}{\Omega_{0}}}$. The parameters $m_{m}$ and $m_{s}$ quantify the severity of multipath fading and shadowing, respectively, in the sense that small values of $m_{m}$ and $m_{s}$ indicate severe multipath fading and shadowing conditions, and vice versa. It can be shown using the moment matching method that $m_{s}=\frac{1}{e^{\left(\sigma_{s} / 8.686\right)^{2}}-1}$ where $\sigma_{s}$ denotes the standard deviation in the lognormal shadowing model

Remark 3.1: The $K$-distribution, where $m_{m}=1$, and a generalized version of it appeared in [46] and [47] to model scattering in radar and laser systems, respectively. Moreover, the generalized- $K$ distribution as introduced in [47] is different from the Gamma-Gamma distribution considered here.

In addition to radar and sonar systems, the generalized- $K$ distribution is used for the modeling atmospheric turbulence in free-space optical communications [48].

The plot of the generalized- $K$ distribution for different values of $m_{m}$ and $m_{s}$ is shown in Fig. 3.2 where the PDF has an exponential shape for severe multipath fading and shadowing conditions, a bell shape for moderate fading conditions, and approaches the Dirac Delta function as the two fading components diminish. 


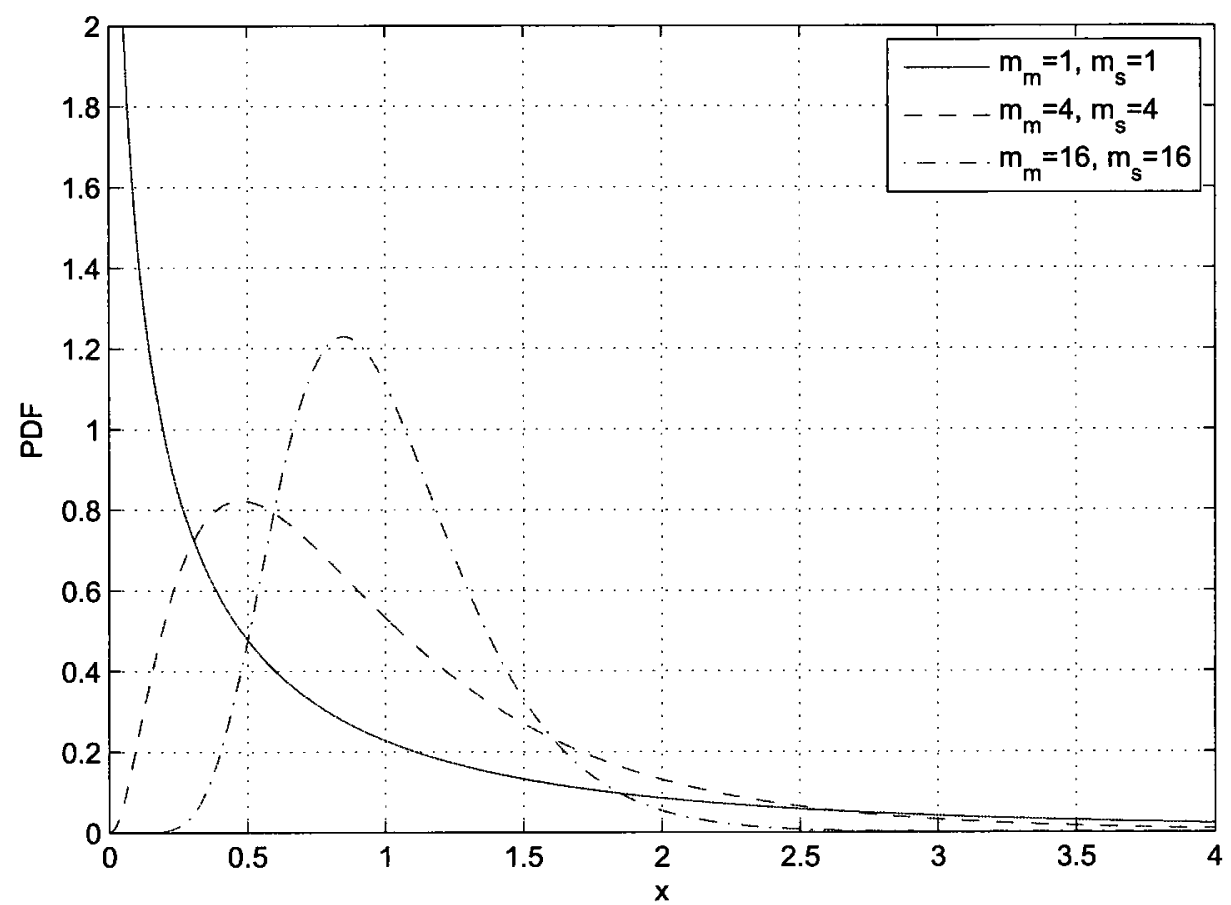

Figure 3.2: The generalized- $K$ distribution plots for different values of $m_{m}$ and $m_{s}$.

The cumulative distribution function (CDF) of $\gamma$ was derived in [49] as

$P(\gamma)=$

$\pi \csc (\pi \alpha)\left[\frac{\left(b^{2} \gamma\right)^{m_{m}}{ }_{1} F_{2}\left(m_{m} ; 1-\alpha, 1+m_{m} ; b^{2} \gamma\right)}{\Gamma\left(m_{s}\right) \Gamma(1-\alpha) \Gamma\left(m_{m}+1\right)}-\frac{\left(b^{2} \gamma\right)^{m_{s}}{ }_{1} F_{2}\left(m_{s} ; 1+\alpha, 1+m_{s} ; b^{2} \gamma\right)}{\Gamma\left(m_{m}\right) \Gamma(1+\alpha) \Gamma\left(m_{s}+1\right)}\right]$,

where $\alpha=m_{s}-m_{m}$ and ${ }_{p} F_{q}$ is the generalized hypergeometric function, [33, Eqn. 9.14.1] for integer $p$ and $q$. Moreover, the characteristic function (CHF) of generalized$K$ RV can be derived as [49]

$$
\Phi_{\gamma}(t)=\left(\frac{b^{2}}{i t}\right)^{\beta / 2} \exp \left(\frac{b^{2}}{2 i t}\right) W_{-\beta / 2, \alpha / 2}\left(\frac{b^{2}}{i t}\right)
$$

where $\beta=m_{m}+m_{s}-1$ and $W_{-\beta / 2, \alpha / 2}(\cdot)$ is the Whittaker function as defined in [33, Eq. 9.220.4].

An alternative representation of the generalized- $K$ distribution can be obtained 
through the use of the general Fox $H$-function, using the fact that the PDF of the product of $N$ Gamma RVs can be expressed as an $H$-function PDF (see Appendix A) [50] that is given as

$$
p_{\gamma}(y)=\left(\prod_{i=1}^{N} \frac{1}{\theta_{i} \Gamma\left(k_{i}\right)}\right) H_{0, N}^{N, 0}\left[\left(\prod_{1}^{N} \frac{1}{\theta_{i}}\right) y \mid\left(k_{i}-1,1\right), \ldots,\left(k_{N}-1,1\right)\right], y>0
$$

Then, the generalized- $K$ PDF can be expressed as

$$
p_{\gamma}(y)=\left(\frac{m_{m} m_{s}}{\Gamma\left(m_{m}\right) \Gamma\left(m_{s}\right) \Omega_{0}}\right) H_{0,2}^{2,0}\left[\left(\frac{m_{m} m_{s}}{\Omega_{0}}\right) y \mid\left(m_{m}-1,1\right),\left(m_{s}-1,1\right)\right], y>0
$$

The expression in (3.17) can be further reduced, using the relation in (A.2) to

$$
p_{\gamma}(y)=\left(\frac{m_{m} m_{s}}{\Gamma\left(m_{m}\right) \Gamma\left(m_{s}\right) \Omega_{0}}\right) G_{0,2}^{2,0}\left[\left(\frac{m_{m} m_{s}}{\Omega_{0}}\right) y \mid m_{m}-1, m_{s}-1\right], y>0
$$

where $G_{p, q}^{m, n}$ denotes the Meijer function [33, Eqn. 9.310]. Furthermore, using [51], the CDF can be expressed as

$$
P(\gamma)=\frac{1}{\Gamma\left(m_{m}\right) \Gamma\left(m_{s}\right)} G_{1,3}^{2,1}\left[\left.\frac{m_{m} m_{s}}{\Omega_{0}} \gamma\right|_{m_{m}, m_{s}, 0} ^{1}\right]
$$

Finally, the CHF can be expressed as

$$
\Phi_{\gamma}(t)=\frac{1}{\Gamma\left(m_{m}\right) \Gamma\left(m_{s}\right)} G_{2,1}^{1,2}\left[-\left.i \frac{\Omega_{0}}{m_{m} m_{s}} t\right|_{0} ^{1-m_{m}, 1-m_{s}}\right]
$$

\subsection{The Approximate Gamma Model}

Although the generalized- $K$ model has led to a closed-form expression for the PDF of the instantaneous power $\gamma$, the use of the corresponding CDF expression which contains the hyper-geometric function term (as in (3.14)) or the Meijer function term (as in (3.19)) is not straightforward due to the associated analytical difficulties and/or 
numerical instabilities that will require the use of approximations and asymptotic expansions. Moreover, further derivations using the characteristic function approach, such as the PDF of the sum of $N$ generalized- $K$ RVs, are quite involved even for the independent and identically distributed (i.i.d.) case due to the difficulties associated with the Whittaker function [52] or the Meijer function as in the CDF expression.

An alternative approach, to avoid these difficulties, is to consider approximating the PDF in (3.13) by a more tractable PDF using the moment-matching method. We propose using the Gamma distribution due to the following reasons: (i) the Gamma distribution is a Type-III Pearson distribution, which is widely used in fitting distributions for positive RVs by matching the first and second moments [50], and (ii) the PDF in (3.13) corresponds to the product of two Gamma RVs and one of the corresponding Gamma PDFs will dominate for large values of $m_{m}$ or $m_{s}$ [53].

\subsubsection{Higher-order Moment Matching}

The $n^{t h}$ moment of the generalized- $K$ distribution can be derived as [52]

$$
E\left[\gamma^{n}\right]=\mu_{n}=\frac{\Gamma\left(m_{m}+n\right) \Gamma\left(m_{s}+n\right)}{\Gamma\left(m_{m}\right) \Gamma\left(m_{s}\right)}\left(\frac{\Omega_{0}}{m_{m} m_{s}}\right)^{n}
$$

where $E[\cdot]$ denotes the statistical expectation.

Furthermore, the $n^{\text {th }}$ moment of the Gamma distribution can be expressed as [29]

$$
E\left[x^{n}\right]=\frac{\Gamma(k+n) \theta^{n}}{\Gamma(k)}
$$

where $k$ and $\theta$ are as defined in (3.7). Now, using the expressions in (3.21) and (3.22), the first, second, and third moments of the generalized- $K$ distribution and the 
approximating Gamma distribution can be matched as

$$
\begin{aligned}
k \theta & =\Omega_{0}, \\
\theta^{2} k(k+1) & =K_{1} \Omega_{0}^{2}, \\
\theta^{3} k(k+1)(k+2) & =K_{2} K_{1} \Omega_{0}^{3} .
\end{aligned}
$$

On the other hand, the negative moments, as defined in [54], of the generalized- $K$ PDF and the Gamma PDF can be expressed using again the expressions in (3.21) and (3.22) as

$$
\begin{gathered}
\theta(k-1)=K_{-1} \Omega_{0}, k>1, m_{m}>1, m_{s}>1, \\
\theta^{2}(k-2)(k-1)=K_{-1} K_{-2} \Omega_{0}^{2}, k>2, m_{m}>2, m_{s}>2,
\end{gathered}
$$

where

$$
\begin{aligned}
K_{1} & =\frac{\left(m_{m}+1\right)\left(m_{s}+1\right)}{m_{m} m_{s}}=1+\frac{1}{m_{m}}+\frac{1}{m_{s}}+\frac{1}{m_{m} m_{s}}, \\
K_{2} & =\frac{\left(m_{m}+2\right)\left(m_{s}+2\right)}{m_{m} m_{s}}=1+\frac{2}{m_{m}}+\frac{2}{m_{s}}+\frac{4}{m_{m} m_{s}}, \\
K_{-1} & =\frac{\left(m_{m}-1\right)\left(m_{s}-1\right)}{m_{m} m_{s}}=1-\frac{1}{m_{m}}-\frac{1}{m_{s}}+\frac{1}{m_{m} m_{s}}, \\
K_{-2} & =\frac{\left(m_{m}-2\right)\left(m_{s}-2\right)}{m_{m} m_{s}}=1-\frac{2}{m_{m}}-\frac{2}{m_{s}}+\frac{4}{m_{m} m_{s}} .
\end{aligned}
$$

Matching different pairs of moments will result in the scale and shape parameters for the approximating Gamma PDF as shown in Table 3.1.

In Table 3.1, $\theta_{i, j}$ and $k_{i, j}$ denote the scale and shape parameters of the approximating Gamma PDF obtained by matching the $i^{\text {th }}$ and the $j^{\text {th }}$ moments, respectively.

Now, examining the expressions of the approximating Gamma PDF parameters given in Table 3.1, the following may be stated:

- The scale parameter of the approximating Gamma PDF obtained by matching the positive moments is larger than the one obtained by matching the negative 
Table 3.1: Expressions of the scale and the shape parameters of the approximating Gamma PDF obtained by moment matching (for $K_{1}, K_{2}, K_{-1}, K_{-2}$, refer to (3.28))

\begin{tabular}{|c|c|c|}
\hline $\begin{array}{l}\text { Moments } \\
\text { matched }\end{array}$ & Scale parameter & Shape parameter \\
\hline$\mu_{1}, \mu_{2}$ & $\theta_{1,2}=\left(K_{1}-1\right) \Omega_{0}, \theta_{1,2}>0$ & $k_{1,2}=\frac{1}{K_{1}-1}, k_{1,2}>0$ \\
\hline$\mu_{1}, \mu_{3}$ & $\theta_{1,3}=\frac{\left(-3+\sqrt{9+8\left(K_{1} K_{2}-1\right)}\right) \Omega_{0}}{4}, \theta_{1,3}>0$ & $k_{1,3}=\frac{4}{-3+\sqrt{9+8\left(K_{1} K_{2}-1\right)}}, k_{1,3}>0$ \\
\hline$\mu_{2}, \mu_{3}$ & $\theta_{2,3}=\sqrt{\frac{K_{1}}{\left(k_{2,3}^{2}+k_{2,3}\right)}} \Omega_{0}, \theta_{2,3}>0$ & $k_{2,3}=\frac{\left(-\frac{K_{2}^{2}}{K_{1}}+4\right)+\sqrt{\left(\frac{K_{2}^{2}}{K_{1}}\right)^{2}+8 \frac{K_{2}^{2}}{K_{1}}}}{2\left(\frac{K_{2}^{2}}{K_{1}}-1\right)}, k_{2,3}>0$ \\
\hline$\mu_{1}, \mu_{-1}$ & $\theta_{1,-1}=\left(1-K_{-1}\right) \Omega_{0}, \theta_{1,-1}>0$ & $k_{1,-1}=\frac{1}{1-K_{-1}}, k_{1,-1}>1$ \\
\hline$\mu_{1}, \mu_{-2}$ & $\theta_{1,-2}=\frac{\left(3-\sqrt{9+8\left(K_{-1} K_{-2}-1\right)}\right) \Omega_{0}}{4}, \theta_{1,-2}>0$ & $k_{1,-2}=\frac{4}{3-\sqrt{9+8\left(K_{-1} K_{-2}-1\right)}}, k_{1,-2}>2$ \\
\hline$\mu_{-1}, \mu_{-2}$ & $\theta_{-1,-2}=\left(\frac{1}{m_{m}}+\frac{1}{m_{s}}-\frac{3}{m_{m} m_{s}}\right) \Omega_{0}, \theta_{-1,-2}>0$ & $k_{-1,-2}=\frac{K_{-1} \Omega_{0}}{\theta_{-1,-2}}+1, k_{-1,-2}>2$ \\
\hline
\end{tabular}

moments. For example, it can be easily seen that $\theta_{1,2}=\theta_{1,-1}+\frac{2}{m_{m} m_{s}} \Omega_{0}$. Since the negative moments characterize a distribution at the origin [54] (the lower tail for a positive RV) and the positive moments characterize a distribution at the upper tail, we may conclude that the generalized- $K$ PDF (CDF) can be approximated by a Gamma distribution whose scale and shape parameters depend on the region of the PDF (CDF) of interest. Such a region-wise (piecewise) approximation was used in [55] to well-approximate the sum of lognormal RVs by a single lognormal RV.

- Matching moments for $n \geq 2$ will lead to involved expressions as seen in Table 3.1. Moreover, not including the first positive moment in the moments matched results in an approximating Gamma PDF that does not have the same mean as the approximated generalized- $K$ PDF (i.e., the generalized- $K$ PDF and the approximating Gamma PDF have different average power values).

- Matching negative moments may not be possible for small values of $m_{m}$ and/or $m_{s}$ as indicated in (3.26) and (3.27) and subsequent expressions in Table 3.1. 

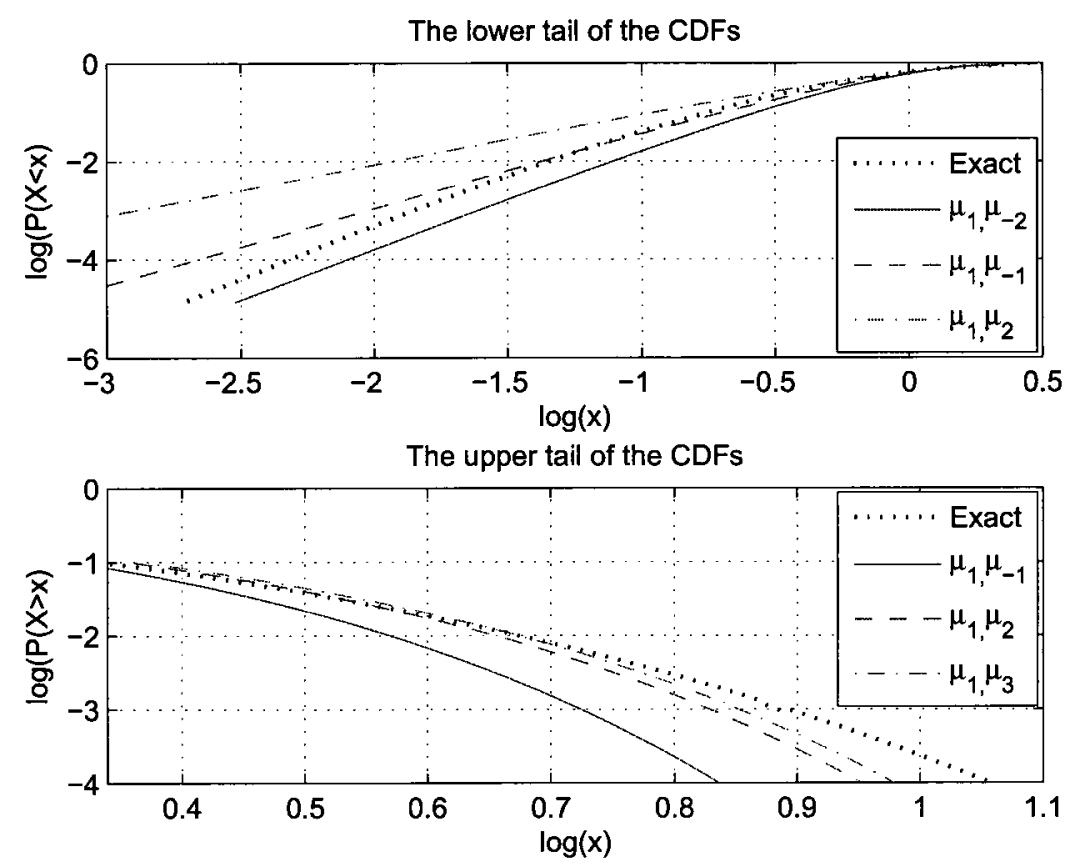

Figure 3.3: The $\log -\log$ CDF plots of the generalized- $K$ and the approximating Gamma RVs for $m_{m}=2.5$ and $m_{s}=2.5$ using the moment matching method.

- The scale and shape parameters of the approximating Gamma distribution are dependent on the fading parameters in the sense that as $m_{m}$ and/or $m_{s}$ increase, the difference between the predicted scale parameters decreases and hence the difference between the approximating PDFs (CDFs) becomes small. So, for small values of $m_{m}$ and/or $m_{s}$ (while $m_{m}, m_{s}>2$ ), the difference between the two approximating Gamma CDFs might be large enough to bound the approximated CDF in the lower tail region as seen in Fig. 3.3. On the other hand, matching the lower order moments for large values of $m_{m}$ and/or $m_{s}$ does not result in a good approximation as seen in Figs. 3.4 and 3.5 since the approximating CDFs are too close to each other.

Note: In Figs. 3.3-5 the complementary cumulative distribution function (CCDF), and particularly the region corresponding to $P(X \geq x) \leq 0.1$, is shown for the upper tail region to obtain more illustrative results. 

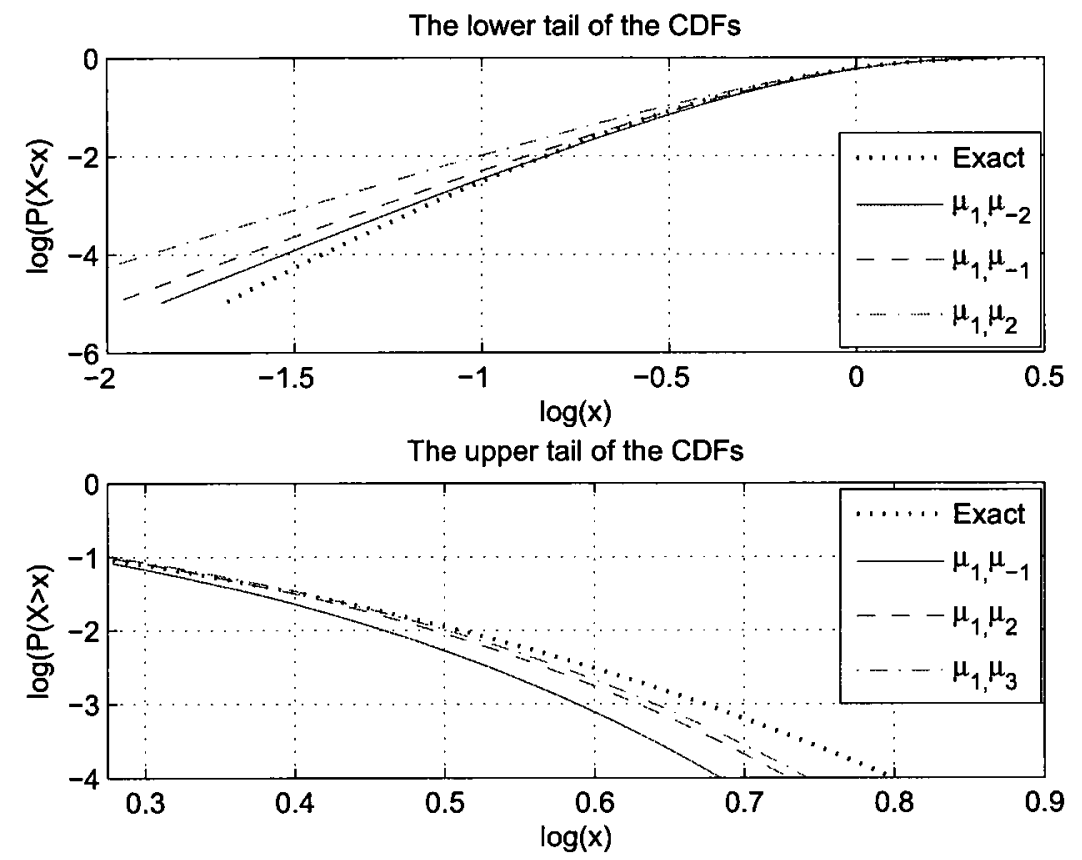

Figure 3.4: The log-log CDF plots of the generalized- $K$ and the approximating Gamma RVs for $m_{m}=7$ and $m_{s}=4$ using the moment matching method.

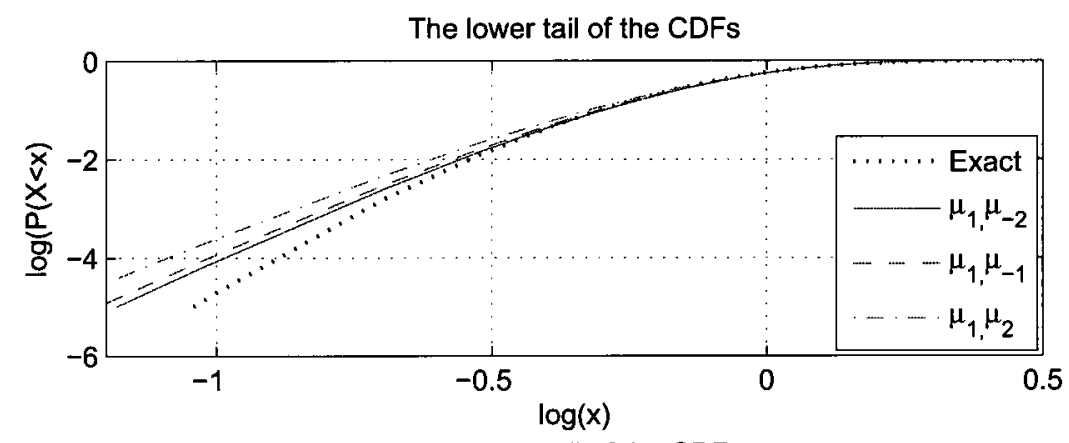

The upper tail of the CDFs

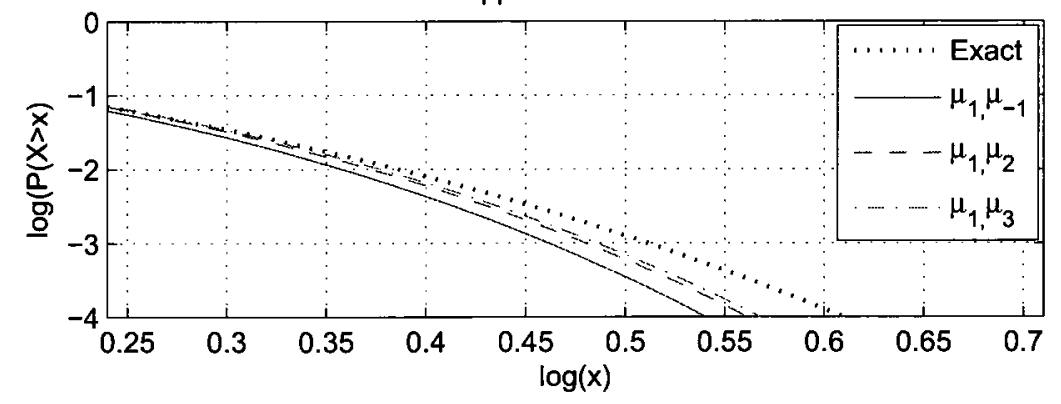

Figure 3.5: The log-log CDF plots of the generalized- $K$ and the approximating Gamma RVs for $m_{m}=10$ and $m_{s}=10$ using the moment matching method. 


\subsubsection{Moment Matching Method with Adjustment}

In order to bypass the limitations explained before on the use of the moment matching for higher order moments, we may consider an adjustable form for the scale and shape parameters of the approximating Gamma PDF obtained by matching only the first two positive moments since (i) these expressions, as given in Table 3.1 , are simple and valid for all values of $m_{m}$ and $m_{s}$; and (ii) the first positive moment is included in the matching. In this regard, we first need to define the following:

Definition 3.1 [56]: The amount of fading (AF) of the instantaneous power $\gamma$ is defined as the ratio of the variance to the square of the mean

$$
\mathrm{AF}_{\gamma}=\frac{\operatorname{var}(\gamma)}{[E(\gamma)]^{2}}
$$

Using the expression in (3.21), the variance of the generalized- $K$ distribution can be expressed as

$$
\operatorname{var}(\gamma)=\frac{\left(m_{m}+m_{s}+1\right) \Omega_{0}^{2}}{m_{m} m_{s}}
$$

Subsequently, the AF can be expressed as

$$
\mathrm{AF}_{\gamma}=\frac{1}{m_{m}}+\frac{1}{m_{s}}+\frac{1}{m_{m} m_{s}}
$$

Now, we may re-write the scale and shape parameters using Table 3.1 as

$$
\begin{aligned}
\theta_{1,2} & =\left[\frac{1}{m_{m}}+\frac{1}{m_{s}}+\frac{1}{m_{m} m_{s}}\right] \Omega_{0}=[\mathrm{AF}] \Omega_{0}, 0 \leq \mathrm{AF} \leq \mathrm{AF}_{\max } \\
k_{1,2} & =\frac{1}{\mathrm{AF}}, 0 \leq \mathrm{AF} \leq \mathrm{AF}_{\max }
\end{aligned}
$$

The value of $A F_{\text {max }}$ is determined by the smallest physical values of $m_{m}$ and $m_{s}$ which are non-zero in real propagation channels; hence $A F_{\text {max }}$ is finite.

The expressions of the scale and shape parameters given by (3.32a) and (3.32b) 


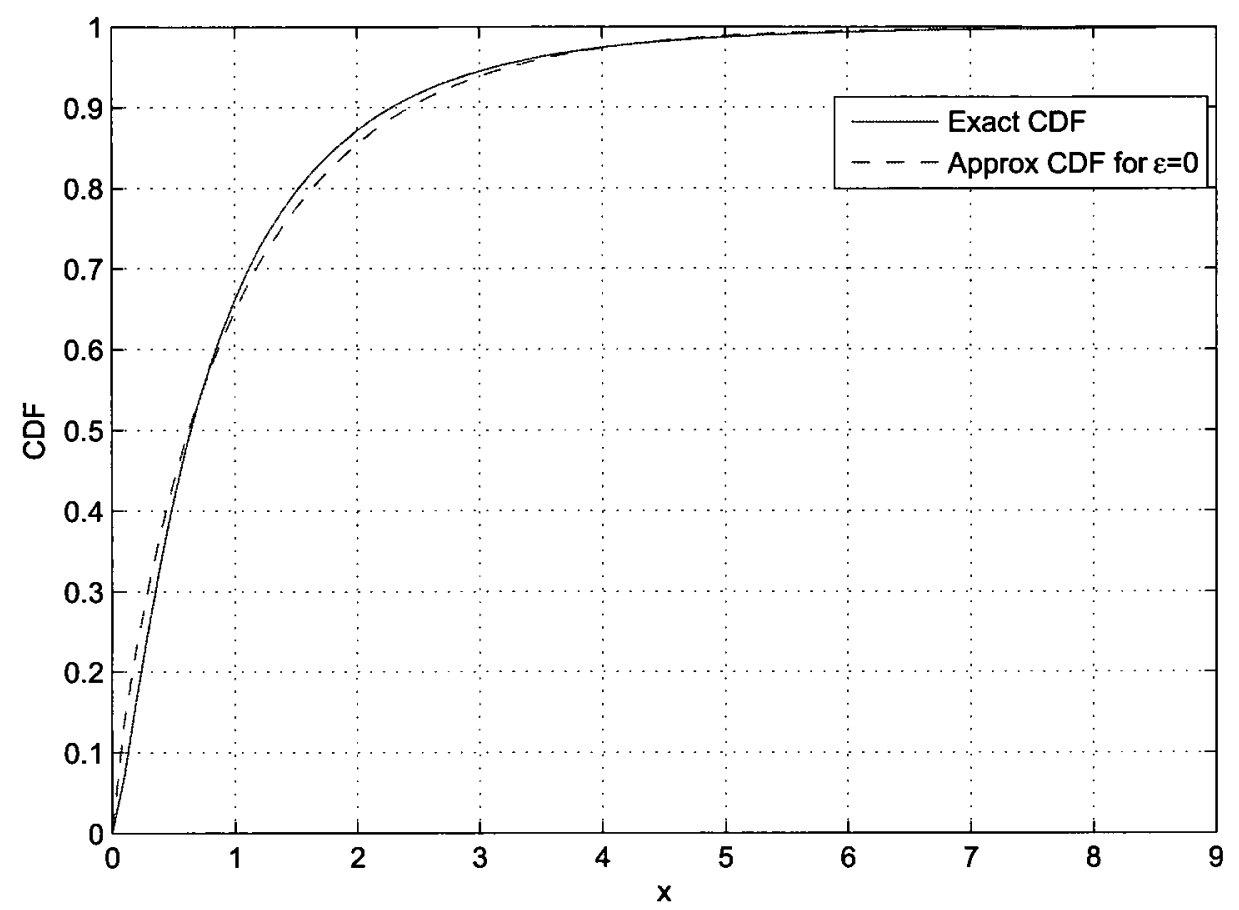

Figure 3.6: The CDF plots of the generalized- $K$ and the approximating Gamma RVs for $m_{m}=2$ and $m_{s}=2$ using the first-two moment matching.

result in a good fit over the main body of the PDF even for small values of $m_{m}$ and $m_{s}$ as shown in Fig. 3.6 for $m_{m}=2$ and $m_{s}=2$. The observation that the CDFs intersects at two points is expected since it was shown in [57] that if two distributions have the same first $n$ moments, then the corresponding CDFs must cross each other at least at $n$ points. However, the approximation is poor in the lower and upper tail regions since matching only the first and second moments will result in a good fit only around the mean ${ }^{2}$.

To overcome this limitation, we may consider the following adjustable form for the expressions in (3.32a) and (3.32b):

$$
\begin{array}{r}
\theta_{1,2}^{\prime}=[\mathrm{AF}-\epsilon] \Omega_{0}, 0 \leq \mathrm{AF} \leq \mathrm{AF}_{\text {max }}, \epsilon_{0} \leq \epsilon \leq \mathrm{AF}, \\
k_{1,2}^{\prime}=\frac{1}{\mathrm{AF}-\epsilon}, 0 \leq \mathrm{AF} \leq \mathrm{AF}_{\text {max }}, \epsilon_{0} \leq \epsilon \leq \mathrm{AF}
\end{array}
$$

\footnotetext{
${ }^{2}$ Theoretical measures such as the Kullback-Leibler distance [60] are analytically difficult to use due to the modified Bessel function term in the generalized- $K$ PDF.
} 
where $\epsilon$ denotes the adjustment factor and $\epsilon_{0}$ denotes its lower limit. Since the AF "added" to the scale parameter of the approximating Gamma PDF should not exceed the original amount of fading of the approximated PDF (i.e., $\epsilon_{0} \geq-\mathrm{AF}$ ), $\epsilon$ is bounded as $-\mathrm{AF} \leq \epsilon \leq \mathrm{AF}$. Due to the fact that the relevant practical range of AF is from zero (for non-fading channels) to 8 (for severe multipath fading and shadowing conditions where $m_{m}=0.5$ and $\left.m_{s}=0.5\right)^{3}$, the relevant range of the adjustment factor $\epsilon$ becomes $-8 \leq \epsilon \leq 8$.

The adjustment factor can be computed using a numerical measure of the difference between the approximated and the approximating PDFs (CDFs). A common measure is the absolute value of the difference between the approximated and the approximating PDFs (CDFs)[29, 59]. For this purpose, the CDFs rather than the PDFs are considered since the Gamma PDF goes to infinity as $x \rightarrow 0$ for $k<1$ [29] which causes numerical instabilities for comparison in the lower tail region.

The plots of the optimal adjustment factor, $\epsilon_{o p}$, versus the multipath fading and shadowing parameters are shown in Figs. 3.7 and 3.8 for values of $m_{m}$ and $m_{s}$ ranging from 0.5 to 10 .

The plots show that the adjustment factor decreases as either or both $m_{m}$ and $m_{s}$ increase. The decrease of the adjustment factor as both $m_{m}$ and $m_{s}$ increase is worth noting since it indicates that the PDF of the product of two Gamma RVs can be wellapproximated, for the main body of the PDF, by a Gamma PDF by matching the first two positive moments. This is due to the fact that both PDFs approach the same limiting PDF (the Dirac delta PDF) as fading diminishes. To see that, the AF for equal values of the multipath fading and shadowing parameters can be expressed as $\mathrm{AF}=\frac{2 m+1}{m^{2}}$, where $m=m_{m}=m_{s}$; clearly the amount of fading is approximately $2 / m$ for moderate values of $m$ and converges to zero for very large values of $m$. However, if a high degree of accuracy is sought in the lower tail region, then the magnitude of

\footnotetext{
${ }^{3}$ Such small values of $m_{m}$ and $m_{s}$ may take place in land mobile satellite channels [58].
} 


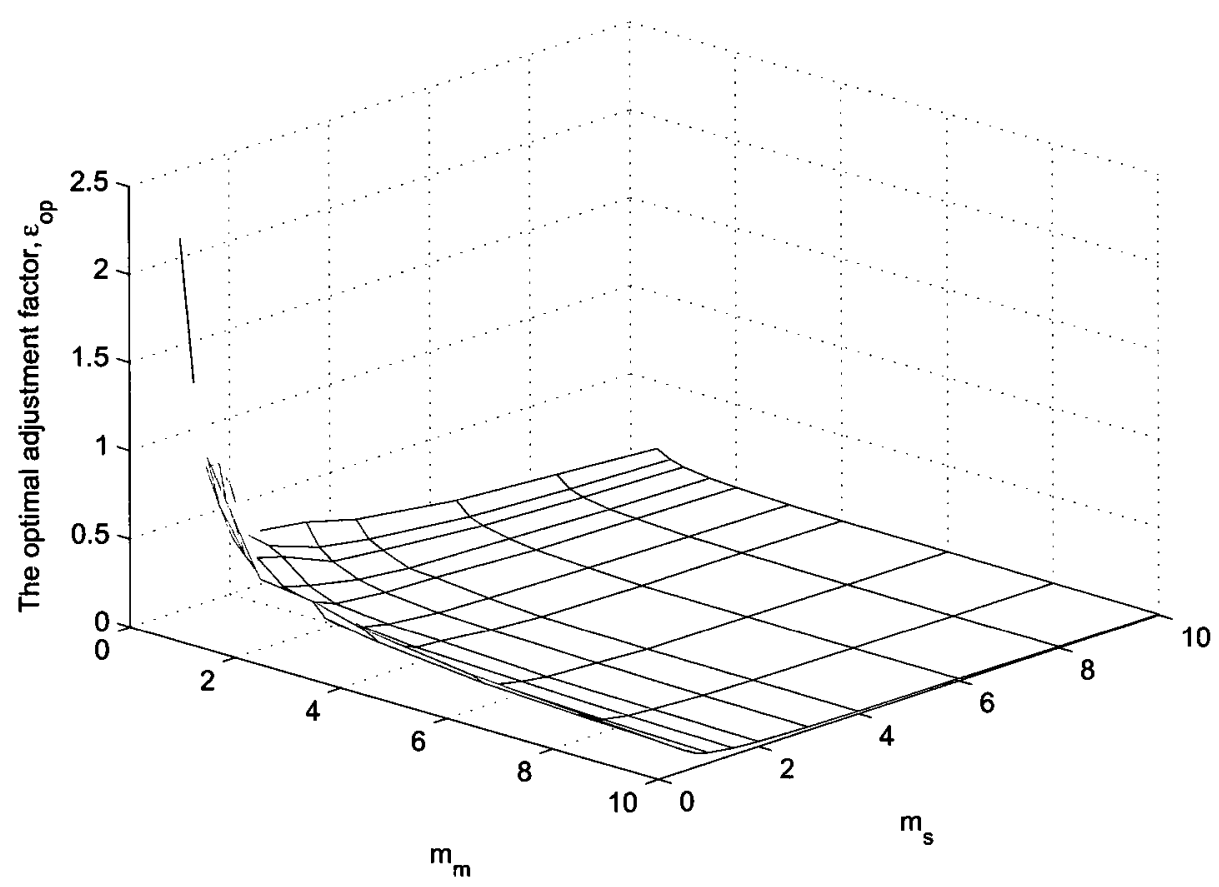

Figure 3.7: The plot of the adjustment factor that minimizes the absolute value of the difference between the approximated generalized- $K$ and the approximating Gamma distributions over the whole CDF.

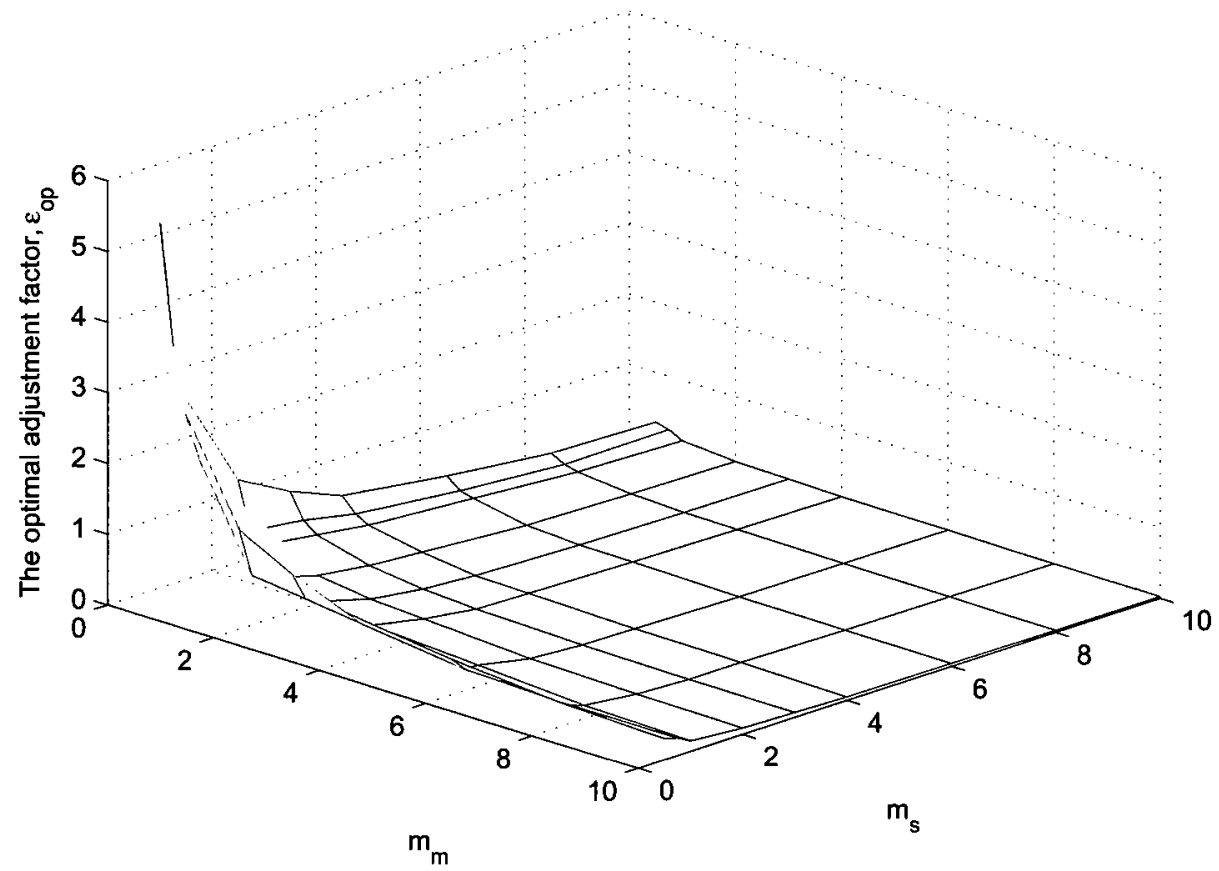

Figure 3.8: The plot of the adjustment factor that minimizes the absolute value of the difference between the generalized- $K$ and the approximating Gamma distributions in the lower tail of the CDF $(<0.1)$. 
the adjustment factor increases as seen in Fig. 3.8. Similar plots can be obtained for any region of interest and the corresponding adjustment factor can be tabulated. The optimal values of the adjustment factor can not be analytically related to the fading and shadowing parameters since the match is performed only over segments of the CDFs which are already analytically complex.

As an example of the applications of the introduced approximation, the ergodic capacity, defined as

$$
C_{e r g}=\int_{0}^{\infty} \log _{2}(1+\operatorname{SNR} x) p_{\gamma}(x) d x
$$

of a heavily shadowed Rayleigh channel $\left(m_{s}=1\right)$ is shown in Fig. 3.9 where the loss in capacity, at high SNR, due to heavy shadowing is $1.66 \mathrm{bits} / \mathrm{s} / \mathrm{Hz}$ as compared to $0.83 \mathrm{bits} / \mathrm{s} / \mathrm{Hz}$ for Rayleigh channels without shadowing [1]. In Fig. 3.9, the value of the adjustment factor (for $m_{s}=1$ ) is chosen, for all SNRs, to be the average of $\epsilon_{o p, 1}$ and $\epsilon_{o p, 0.1}$ (corresponding to the lower one-tenth portion of the CDF); i.e., $\epsilon=\left(\epsilon_{o p, 1}+\epsilon_{o p, 0.1}\right) / 2[61]$.

\subsection{Conclusions}

In this chapter, an introduction about the existing multipath fading, shadowing, and composite fading models is given, then, particularly, the generalized- $K$ model is presented and its relation to the $H$-function distribution family is highlighted. The approximation of the generalized- $K$ distribution by the Gamma distribution using higher-order moments is investigated and subsequently an adjustable form of the first two moment-matching method is proposed for region-wise approximation. The introduced approximation leads to simple approximate closed-form expressions for the ergodic capacity and the information outage probability and allows approximating the diversity gain of diversity combining over generalized- $K$ channels. 


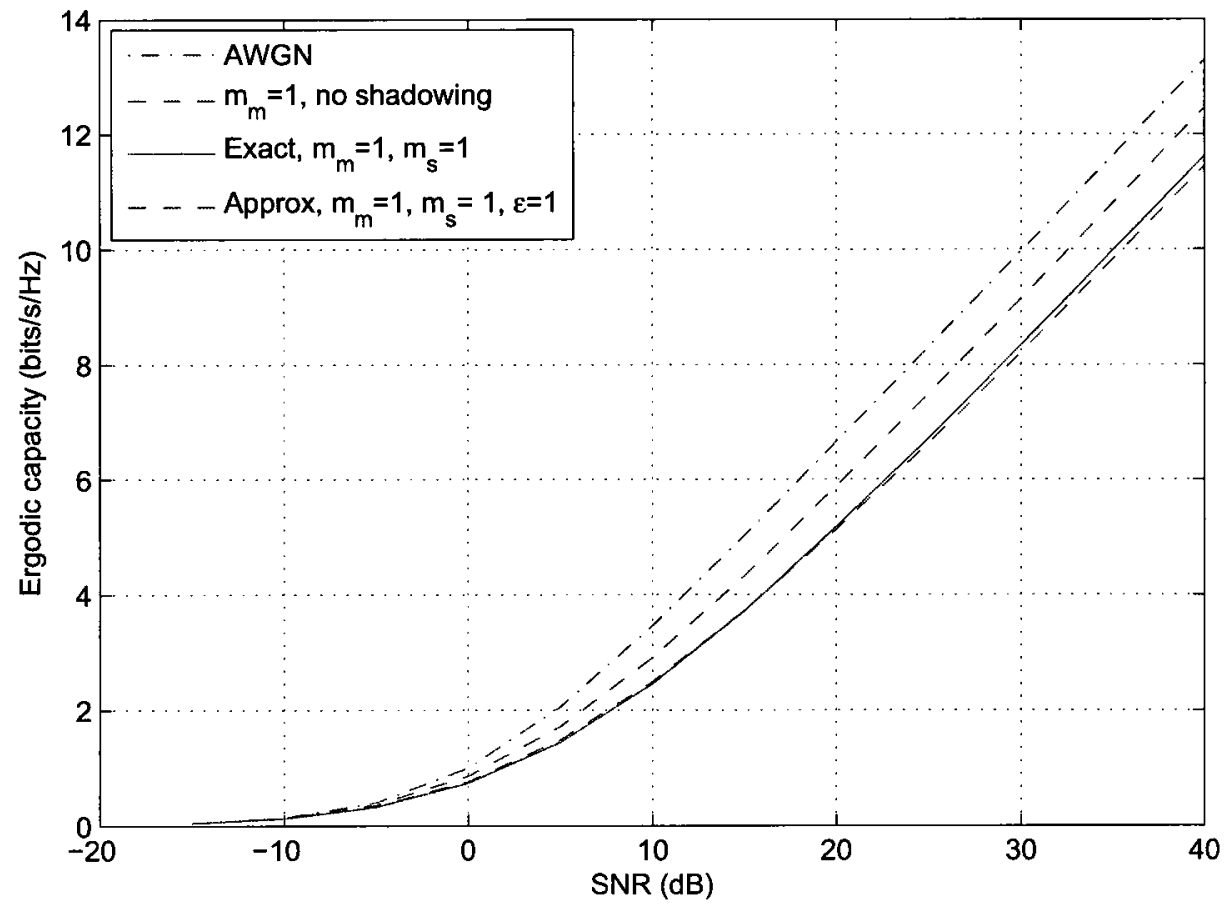

Figure 3.9: The ergodic capacity plot of a Rayleigh fading channel with and without shadowing. 


\section{Chapter 4}

\section{On the Statistics of the Sum of Generalized- $K$ RVs}

The determination of the distribution of the sum of generalized- $K \mathrm{RVs}$ is of relevance since the performance analysis of several communication schemes is determined or lower/upper-bounded using the sum statistics. In this chapter, the statistics of the sum of generalized- $K \mathrm{RVs}$ is considered for both the independent and correlated cases. For the independent case, the sum distribution is approximated by (i) a Gamma distribution based on the region-wise approximation introduced in Chapter 3 and (ii) another generalized- $K$ PDF whose parameters are determined again by the moment matching method. For the correlated case, the second-order statistics are considered and expressions for the AF are developed.

\subsection{Related Work}

Early work on the sum of independent $K$-distributed RVs has appeared in [62] where the CHF approach was used to derive the sum distribution. This result was extended in [52] for the sum distribution of generalized- $K$ RVs for integer values of $m_{m}$ and $m_{s}$. However, the expressions of the PDF of the sum distribution turned out to be complex even for the independent and identically distributed (i.i.d.) case with integer parameters. In [48], an infinite series expansion of the Bessel function term in the 
generalized- $K$ PDF was used to express the PDF for the sum of i.i.d. generalized- $K$ RVs in the context of the performance analysis of optical communication systems. The power series are truncated to a finite number of terms and the BER of certain modulation schemes for both single-input single-output (SISO) and MIMO channels. In [63], The moment generating function (MGF) approach, introduced in [64], was used to derive closed-form expressions for the BER of binary differential phase-shift keying and orthogonal binary frequency-shift keying (FSK) modulation over multibranch over generalized- $K$ fading channels. The formulation is based on expressing the Whittaker function in the MGF expression using the more familiar confluent hypergeometric function of the second kind and then deriving the BER expressions. For the correlated case, results were reported for the bivariate case only, in [65], where the PDF, the CDF, and the CHF of the joint distribution are derived in infinite series form and the performance of maximal ratio combining (MRC) and equal gain combining for a dual diversity combiner are studied.

\subsection{The Approximate Gamma Distribution for the Sum of Independent Generalized- $K$ RVs}

The moment matching method can be used to approximate the PDF (CDF) of the sum of $N$ generalized- $K$ RVs by a Gamma PDF (CDF). However, matching the higher order moments is difficult since deriving or computing these moments is involved or unfeasible $[54,55]$. This motivates again the use of an adjustable form for the scale and shape parameters obtained by matching the first two positive moments.

We may start with $N=2$ where the first and second moments of the sum of two independent RVs, $z=x+y$, can be expressed as [29]

$$
E[z]=E[x]+E[y]
$$


and

$$
E\left[z^{2}\right]=E\left[x^{2}\right]+E\left[y^{2}\right]+2 E[x] E[y]
$$

Matching the first and second moments of the sum of two independent generalized- $K$ RVs and the approximating Gamma distribution results in

$$
\begin{aligned}
\theta_{\text {sum }}= & \frac{K_{1, x} \Omega_{0, x}^{2}+K_{1, y} \Omega_{0, y}^{2}+2\left(\Omega_{0, x} \Omega_{0, y}\right)-\left(\Omega_{0, x}+\Omega_{0, y}\right)^{2}}{\left(\Omega_{0, x}+\Omega_{0, y}\right)} \\
= & \frac{\mathrm{AF}_{x} \Omega_{0, x}^{2}+\mathrm{AF}_{y} \Omega_{0, y}^{2}}{\left(\Omega_{0, x}+\Omega_{0, y}\right)}, \theta_{\text {sum }}>0
\end{aligned}
$$

and

$$
k_{\text {sum }}=\frac{\left(\Omega_{0, x}+\Omega_{0, y}\right)^{2}}{\mathrm{AF}_{x} \Omega_{0, x}^{2}+\mathrm{AF}_{y} \Omega_{0, y}^{2}}, k_{\text {sum }}>0,
$$

where $K_{1, x}$ and $K_{1, y}$ denote the $K_{1}$ parameters (as defined in $(3.27 \mathrm{a})$ ), $\Omega_{0, x}$ and $\Omega_{0, y}$ denote the values of the mean of the local power, and $\mathrm{AF}_{x}$ and $\mathrm{AF}_{y}$ denote the $\mathrm{AF}$ of the generalized- $K \mathrm{RVs} x$ and $y$, respectively.

The adjusted forms of $(4.2 \mathrm{a})$ and $(4.2 \mathrm{~b})$ can be written as

$$
\theta_{\text {sum }}^{\prime}=\frac{\left[\mathrm{AF}_{x}-\epsilon_{x}\right] \Omega_{0, x}^{2}+\left[\mathrm{AF}_{y}-\epsilon_{y}\right] \Omega_{0, y}^{2}}{\left(\Omega_{0, x}+\Omega_{0, y}\right)}, \theta_{\text {sum }}^{\prime}>0
$$

and

$$
k_{\text {sum }}^{\prime}=\frac{\left(\Omega_{0, x}+\Omega_{0, y}\right)^{2}}{\left[\mathrm{AF}_{x}-\epsilon_{x}\right] \Omega_{0, x}^{2}+\left[\mathrm{AF}_{y}-\epsilon_{y}\right] \Omega_{0, y}^{2}}, k_{\text {sum }}^{\prime}>0 .
$$

In general, the expressions in (4.3a) and (4.3b) can be generalized for the sum of $N$ independent generalized- $K \mathrm{RVs}$ as

$$
\theta_{\text {sum }}^{\prime}=\frac{\sum_{i=1}^{N}\left[\mathrm{AF}_{i}-\epsilon_{i}\right] \Omega_{0, i}^{2}}{\sum_{i=1}^{N} \Omega_{0, i}}, \theta_{\text {sum }}^{\prime}>0
$$

and

$$
k_{\text {sum }}^{\prime}=\frac{\left(\sum_{i=1}^{N} \Omega_{0, i}\right)^{2}}{\sum_{i=1}^{N}\left[\mathrm{~A} F_{i}-\epsilon_{i}\right] \Omega_{0, i}^{2}}, k_{s u m}^{\prime}>0 .
$$


For the i.i.d. case, the expressions in (4.4a) and (4.4b) simplify to

$$
\theta_{\text {sum }}^{\prime}=(\mathrm{AF}-\epsilon) \Omega_{0}, \theta_{\text {sum }}^{\prime}>0
$$

and

$$
k_{\text {sum }}^{\prime}=\frac{N}{\mathrm{AF}-\epsilon}, k_{\text {sum }}^{\prime}>0 .
$$

Similar formulation can be carried out for the sum of correlated generalized- $K$ RVs.

Three-dimensional plots of the adjustment factor versus the composite fading parameters $m_{m}$ and $m_{s}$ can be produced for different values of $N$. As an example, the plots of the lower tail of the CDFs for $m_{m}=2, m_{s}=4$, and $N=1,2,3$, and 6 are given in Fig. 4.1 showing that an adjustment factor of $\epsilon=0.2$ results in almost identical CDFs, in the lower tail region, for $N=6$. Clearly, larger values of $\epsilon$ are needed for a more accurate approximation for $N=1,2$, and 3 . The fit improves for large $N$ due to the observation that the Gamma distribution (being approximately Gaussian for large values of the shape parameter) approximates the distribution of the sum of independent positive RV especially if they have a moderately slow decaying upper tail characteristics. This would explain why the accuracy does not degrade as the number of summands increases.

Remark 4.1: Another approach to approximate the PDF of the sum of independent generalized- $K$ RVs can be based on the fact that the lower and upper tails of the PDF of the sum of independent positive RVs are due to the convolution of the lower and upper tails of the corresponding individual PDFs, respectively. So, the results obtained in Section 3.3.2 can be used to approximate the PDF of the sum of $N$ i.i.d. generalized- $K$ RVs by the PDF of the sum of the approximating $N$ i.i.d. Gamma RVs. It is well-known that the sum of $N$ i.i.d. Gamma RVs, with the same shape and scale parameters $k_{1,2}^{\prime}$ and $\theta_{1,2}^{\prime}$, respectively, is another Gamma RV whose shape and scale parameters are $N k_{1,2}^{\prime}$ and $\theta_{1,2}^{\prime}$, respectively; these are the same as the ones obtained 


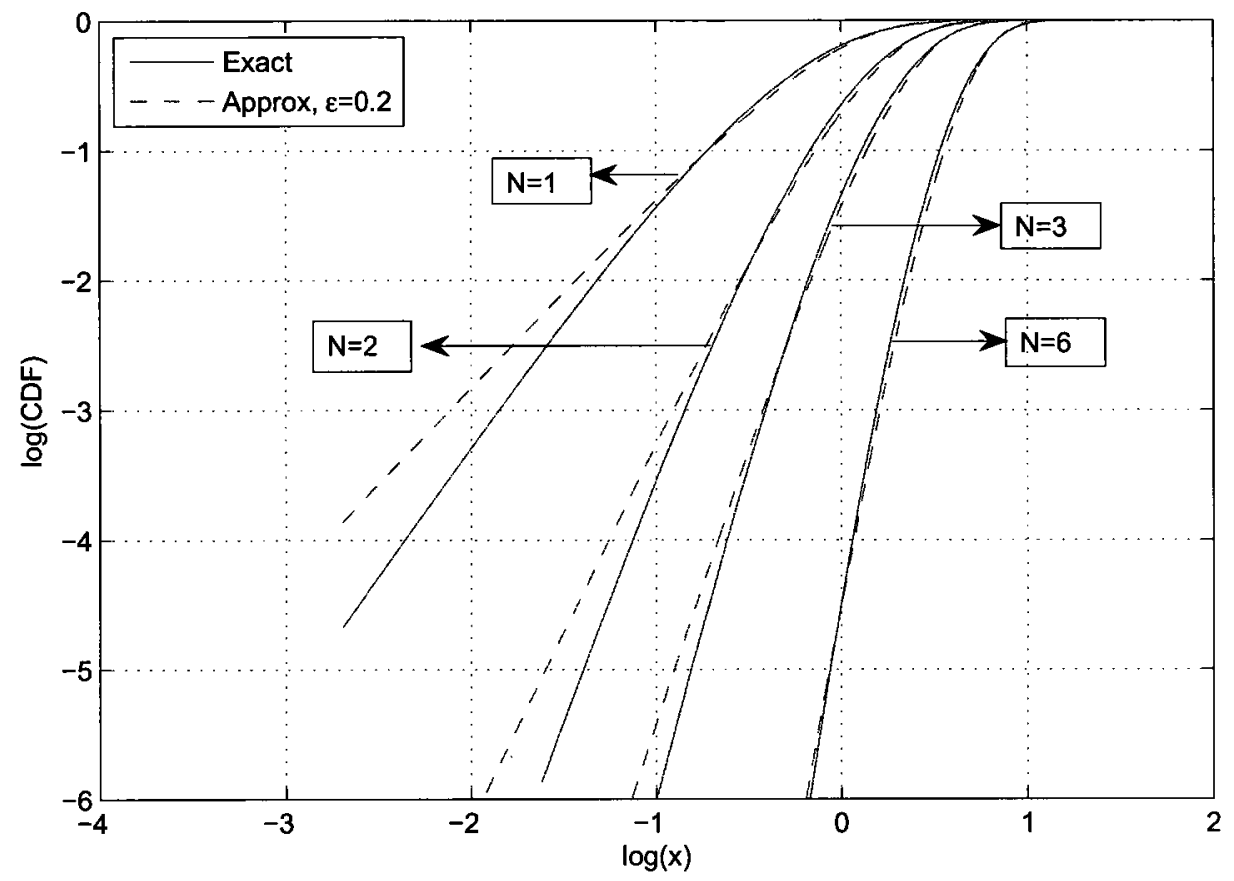

Figure 4.1: The log-log CDF plots for the sum of generalized- $K$ RVs and the approximating Gamma RV for $m_{m}=2, m_{s}=4\left(\sigma_{s}=4.2 \mathrm{~dB}\right), \epsilon=0.2$, and different values of $N$.

in (4.5a) and (4.5b). For the non-identically distributed case, the existing results in literature on the distribution of the sum of independent non-identically distributed Gamma RVs can be utilized $[66,67]$.

This approximation was used in [61] to well-approximate the ergodic capacity (as defined in (3.34)) of MRC receivers over independent generalized- $K$ channels. Furthermore, the approximate Gamma model allows approximating the diversity gain as the AF of the approximate model [68]. 


\subsection{The Approximate Generalized- $K$ PDF for the PDF of the Sum of Independent Generalized- $K \mathbf{R V s}$}

Let $\zeta$ denote the sum of $N$ independent generalized- $K \mathrm{RVs}$, as

$$
\zeta=z_{1} w_{1}+z_{2} w_{2}+\ldots+z_{N} w_{N}
$$

where $z_{i}$ and $w_{i}$ represent the mutually independent multipath fading and shadowing RVs, respectively. In general, the PDF of $\zeta$ can be obtained by convolving the individual PDFs of $z_{i} w_{i}^{i}$ s or by using the CHF approach. In [62], the CHF approach was used to derive the PDF of the sum of i.i.d. $K$-distributed RVs. This result was extended in [52] to derive the PDF of the sum of i.i.d. generalized- $K$ RVs for integer values of $m_{m}$ and $m_{s}$. However, due to the encountered analytical difficulties and numerical instabilities associated with the Whittaker function term or the Meijer function term in the CHF of the generalized- $K$ distribution, the PDF of the sum of $N$ independent generalized- $K$ RVs becomes cumbersome to derive for cases other than that limiting one. As an alternative, we may consider approximating the PDF of $\zeta$ by another generalized- $K \mathrm{RV}$ inspired by the observation that a generalized- $K$ RV for large values of $m_{m}$ and/or $m_{s}$ can be well-approximated by a Gamma RV [53] and it is well-known that the sum of such independent Gamma RVs is another Gamma RV [29] (i.e., a generalized- $K$ RV with large $m_{m}$ and/or $m_{s}$ values).

\subsubsection{Case of i.i.d. Generalized- $K$ RVs}

The PDF of the sum of $N$ i.i.d. generalized- $K$ RVs can be closely approximated by another generalized- $K$ PDF as will be explained next.

Proposition 4.1: The PDF of the sum of $N$ i.i.d. generalized- $K$ RVs $\left(m_{m, 1}=\right.$ 
$m_{m, 2}=\ldots=m_{m, N}=m_{m}, m_{s, 1}=m_{s, 2}=. .=m_{s, N}=m_{s}$, and $\Omega_{0,1}=\Omega_{0,2}=\ldots .=$ $\Omega_{0, N}=\Omega_{0}$ ) can be approximated by the PDF of an equivalent generalized- $K \mathrm{RV}, \tilde{\zeta}$,

$$
p_{\tilde{\zeta}}(x)=\frac{2 b_{\tilde{\zeta}}^{m_{m, \tilde{\zeta}}+m_{s, \tilde{\zeta}}}}{\Gamma\left(m_{m, \tilde{\zeta}}\right) \Gamma\left(m_{s, \tilde{\zeta}}\right)} x^{\left(m_{m, \tilde{\zeta}}+m_{s, \tilde{\zeta}}\right) / 2-1} K_{m_{s, \tilde{\zeta}}-m_{m, \tilde{\zeta}}}\left(2 b_{\tilde{\zeta}} \sqrt{x}\right)
$$

where $b_{\tilde{\zeta}}=\sqrt{\frac{m_{m, \tilde{\zeta}} m_{s, \tilde{\zeta}}}{\Omega_{0, \tilde{\zeta}}}}$ and

$$
\begin{gathered}
m_{m, \tilde{\zeta}}=\frac{(1+a)+\sqrt{(1+a)^{2}+\frac{4}{N m_{s}^{2}} k_{1}}}{2\left(\frac{m_{m}}{m_{s}}+\frac{1}{m_{s}}+1\right)} m_{m} N \\
m_{s, \tilde{\zeta}}=\frac{m_{m, \tilde{\zeta}}}{a}, \\
\Omega_{0, \tilde{\zeta}}=N \Omega_{0},
\end{gathered}
$$

where $k_{1}=\left(m_{m}+m_{s}+1\right)$ and $a=\frac{m_{m}}{m_{s}}$. The expression in (4.8a) simplifies, for the case of $a=1$, to

$$
m_{m, \tilde{\zeta}}=\frac{1+\sqrt{1+\frac{1}{N m^{2}}(2 m+1)}}{2+\frac{1}{m}} m N
$$

where $m_{m}=m_{s}=m$.

Proof: The proof can be divided into two steps. The first step is to observe that the AF of the sum of $N$ i.i.d. generalized- $K \mathrm{RVs}$, as in (4.6), is given as $\mathrm{AF}_{\zeta}=$ $\frac{1}{N m_{m}}+\frac{1}{N m_{s}}+\frac{1}{N m_{m} m_{s}}$. This can be seen simply by considering the fact that the variance of the sum of $N$ i.i.d. RVs scales with $N$ and the square of the mean scales with $N^{2}$; hence the AF of the sum of $N$ i.i.d. generalized- $K$ RVs will be equal to the $\mathrm{AF}$ of each individual $\mathrm{RV}$ in the sum, as given in (3.31), scaled by $1 / N$. The second step is to obtain the parameters of an equivalent generalized- $K$ PDF whose AF is given as $A F_{\tilde{\zeta}}=A F_{\zeta}$. This can be done by equating the AF of the approximating 
generalized- $K \mathrm{RV}$ to $\mathrm{AF}_{\zeta}$ as

$\mathrm{AF}_{\tilde{\zeta}}=\frac{1}{m_{m, \tilde{\zeta}}}+\frac{1}{m_{s, \tilde{\zeta}}}+\frac{1}{m_{m, \tilde{\zeta}} m_{s, \tilde{\zeta}}}=\frac{1}{m_{m, \tilde{\zeta}}}+\frac{a_{\tilde{\zeta}}}{m_{m, \tilde{\zeta}}}+\frac{a_{\tilde{\zeta}}}{m_{m, \tilde{\zeta}}^{2}}=\frac{1}{N m_{m}}+\frac{1}{N m_{s}}+\frac{1}{N m_{m} m_{s}}$

which can be solved for $m_{m, \zeta}$ to get

$$
m_{m, \tilde{\zeta}}=\frac{m_{m} m_{s}\left(\left(1+a_{\tilde{\zeta}}\right)+\sqrt{\left(1+a_{\tilde{\zeta}}\right)^{2}+\frac{4}{N m_{m} m_{s}} k_{1} a_{\tilde{\zeta}}}\right)}{2\left(m_{m}+m_{s}+1\right)} N
$$

where $a_{\tilde{\zeta}}=\frac{m_{m, \tilde{\zeta}}}{m_{s, \tilde{\zeta}}}$. We may assume $a_{\tilde{\zeta}}=a$ to get the first two expressions in (4.8a) and (4.8b). The use of this assumption is based on the intuition that the sum of $N$ i.i.d. generalized- $K \mathrm{RVs}$ (each having $m_{m}=a m_{s}$ ) is more likely to be approximated by another generalized- $K \mathrm{RVs}$ with $m_{m, \tilde{\zeta}}=a m_{s, \tilde{\zeta}}$. For example, if the ratio, $a$, is much larger or much smaller than unity, then each individual generalized- $K$ RV in the sum is approximately a Gamma RV [53], and the sum of these i.i.d. Gamma RVs is another Gamma RV [29]. The expression in (4.8c) is obtained in a straightforward way by matching the first moments of $\zeta$ and $\tilde{\zeta}$.

Remark 4.2: The case where the shadowing effect diminishes $\left(m_{s} \rightarrow \infty\right)$ will result in $m_{m, \zeta}=N m_{m}$ as expected, since the Gamma PDF corresponding to the shadowing component approaches a Dirac Delta PDF and the composite fading PDF will reduce to the Gamma PDF corresponding to the multipath fading. Furthermore, it is well-known that the PDF of the sum of i.i.d. Gamma RVs is another Gamma PDF whose shape parameter is the sum of their individual shape parameters [29].

Remark 4.3: An alternative approach to solve for $m_{m, \tilde{\zeta}}$ and $m_{s, \tilde{\zeta}}$ is to consider matching the third-order moment of $\zeta$ and $\tilde{\zeta}$ (as a third equation); however, the resulting expressions will be too involved to draw insights from, and will result in fitting characteristics that are biased towards the upper tail region which can be seen, for example, in [69]. 


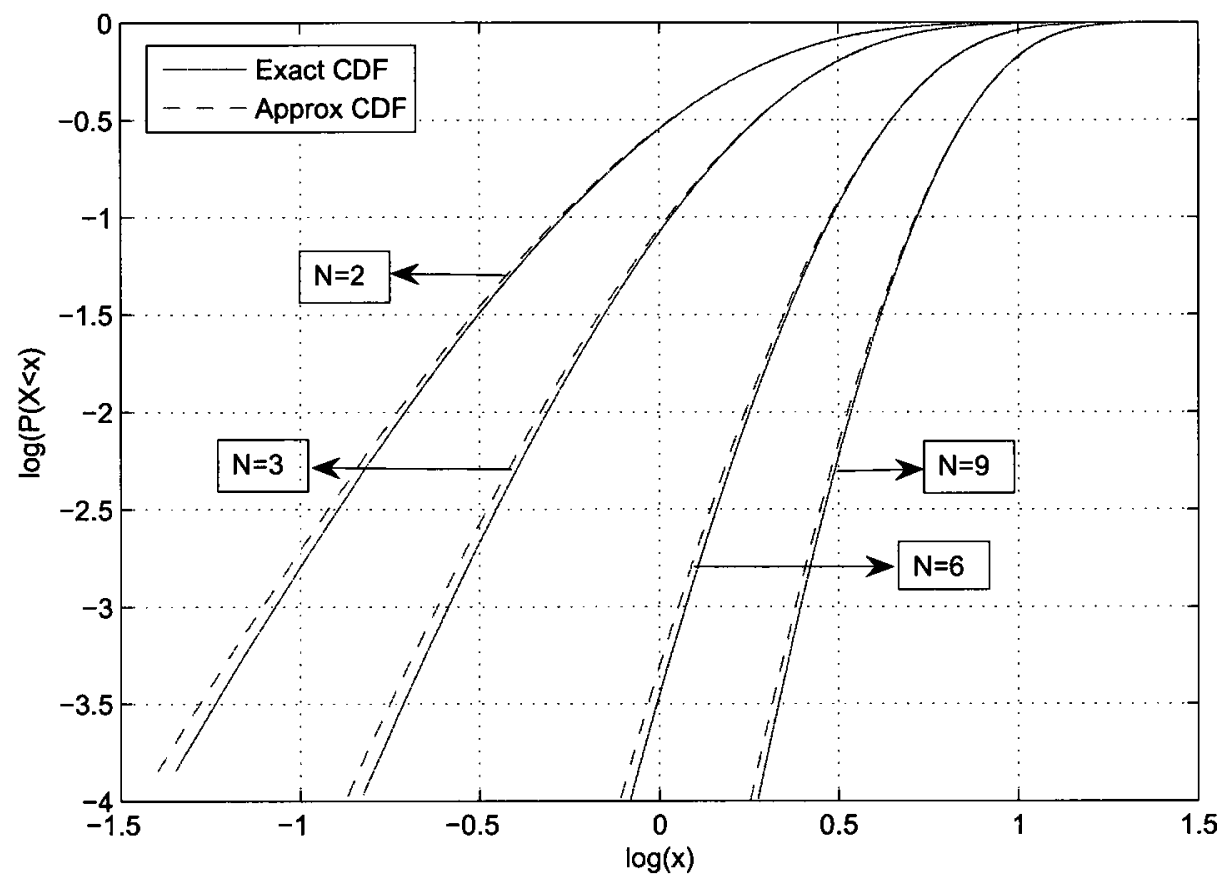

Figure 4.2: The log-log (to base 10) CDF plots for the lower tail of the sum of generalized- $K$ RVs and the approximating generalized- $K$ RV for different values of $N$ with $m_{m}=m_{s}=2$.

Remark 4.4: In an independent work [70], the sum distribution in (4.6) is approximated by another generalized- $K$ distribution whose parameters are given as $m_{m, \tilde{\zeta}}=N m_{m}$ and $m_{s, \tilde{\zeta}}=N m_{s}-\varepsilon$ where the adjustment parameter, $\varepsilon$, is determined numerically. The expressions in (4.8a) and (4.8b), are obtained using a more systematic approach and lead to sufficiently accurate approximation, as shown in the next figures, that require introducing an adjustment parameter only for small values of $m_{m}, m_{s}$, and $N$.

To demonstrate the attained accuracy by the proposed approximation, we may first consider the i.i.d. case where the CDF plots of the approximated sum distribution, obtained by convolving the individual PDFs and the approximating generalized$K$ distribution whose parameters are given by (4.8a)-(4.8c), for $m_{m}=m_{s}=2$ and $m_{m}=m_{s}=4$ are shown in Figs. 4.2-4, respectively.

The plots show that the approximation accuracy improves as $m_{m}$ and $m_{s}$ increases 


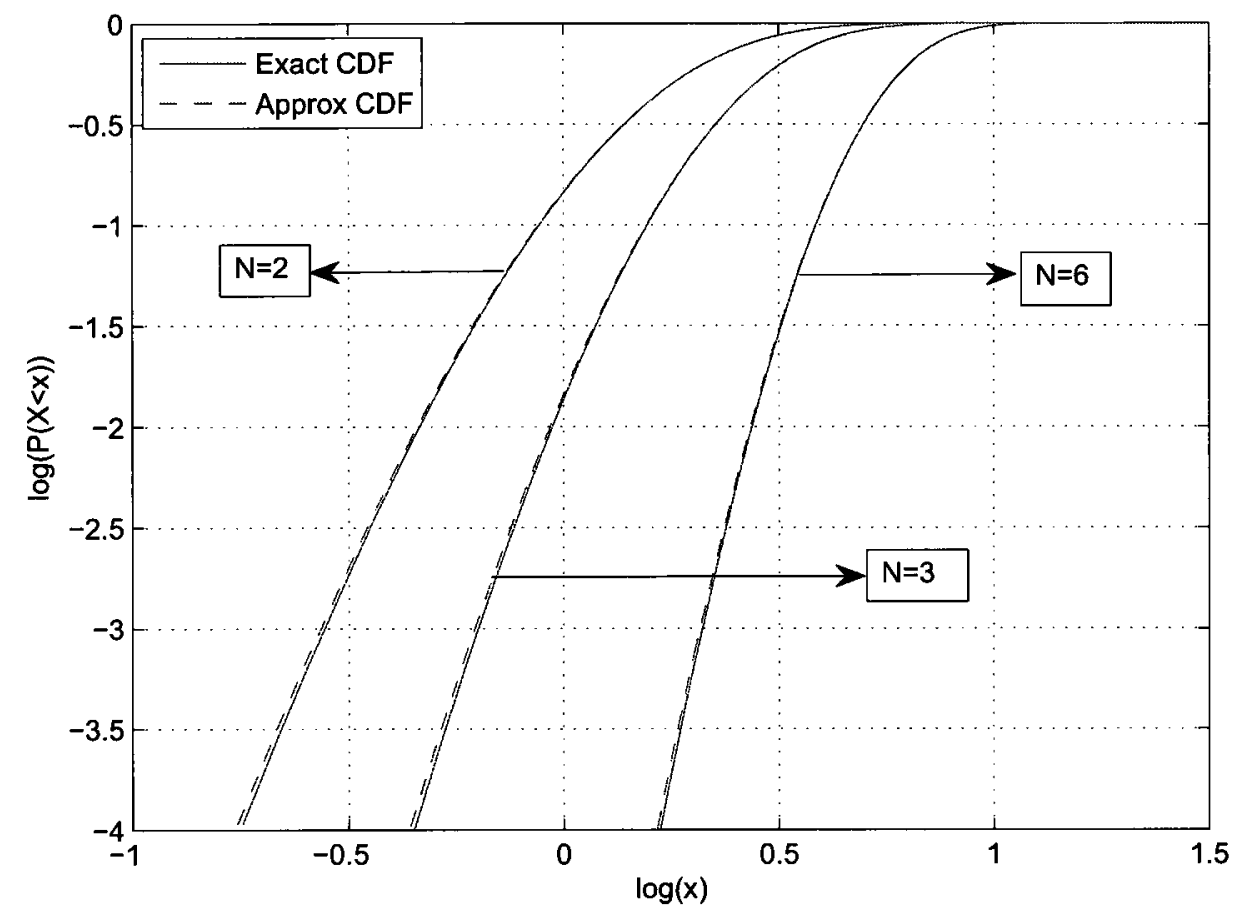

Figure 4.3: The log-log (to base 10) CDF plots for the lower tail of the sum of generalized- $K \mathrm{RVs}$ and the approximating generalized- $K \mathrm{RV}$ for different values of $N$ with $m_{m}=m_{s}=4$.

and that the fit is tight for both the lower and upper tail regions. As the multipath fading and the shadowing parameters increase, the PDF of each of the summands gets less asymmetric and more "Gamma-like" so that the accuracy of the approximation based on the first two moments and the additional rule $\left(m_{m, \tilde{\zeta}}=a m_{s, \tilde{\zeta}}\right)$ improves. Figure 4.5 shows the CDF plots for the sum of i.i.d. generalized- $K$ RVs with $m_{m}=3$ and $m_{s}=6$ and the approximating generalized- $K$ RV whose parameters can be computed using (4.8a) and (4.8b) as $m_{m, \tilde{\zeta}}=5.72$ and $m_{s, \tilde{\zeta}}=11.44$ for $N=2, m_{m, \tilde{\zeta}}=$ 8.42 and $m_{s, \tilde{\zeta}}=16.84$ for $N=3$, and $m_{m, \tilde{\zeta}}=16.53$ and $m_{s, \tilde{\zeta}}=33.06$ for $N=6$.

The plots in Figs. 4.2-5 indicate that the PDF of $\zeta$ is actually well-approximated by another generalized- $K$ PDF for different values of $m_{m}$ and $m_{s}$ in both the lower and upper tail regions. To verify the accuracy of the obtained results, the values of $m_{m, \tilde{\zeta}}$ and $m_{s, \tilde{\zeta}}$ of the approximating generalized- $K$ PDF (for $m_{m}=m_{s}=4$ ) that result in the optimal least-square fit to the sum PDF, can be computed using the 


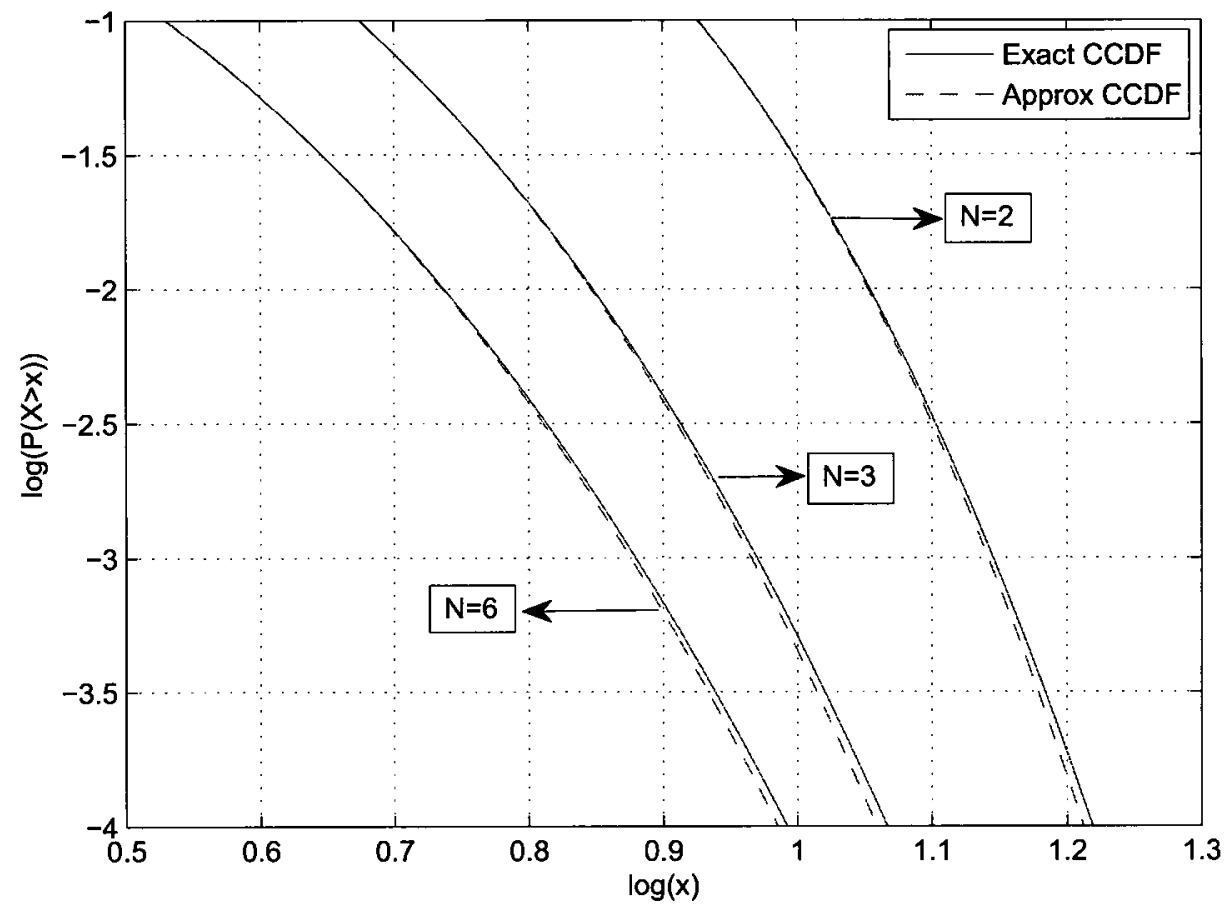

Figure 4.4: The log-log (to base 10) CDF plots for the upper tail of the sum of generalized- $K$ RVs and the approximating generalized- $K$ RV for different values of $N$ with $m_{m}=m_{s}=4$.

MATLAB function "Isqcurvefit" as 7.63 and 7.64, 11.18 and 11.28, and 21.97 and 21.96 , for $N=2,3$, and 6 , respectively, as compared to $7.58,11.14$, and 21.82 , for $N=2,3$, and 6 , respectively.

Remark 4.5: To tighten the fit between the approximated and approximating PDFs (CDFs), we may introduce a parameter $\epsilon$, such that $\tilde{m}_{m, \tilde{\zeta}}=m_{m, \tilde{\zeta}}+\tilde{\epsilon_{m}}$ and $\tilde{m}_{s, \tilde{\zeta}}=m_{s, \tilde{\zeta}}+\tilde{\epsilon}_{s}$, as an adjustment parameter to account for the remaining inaccuracy in $m_{m, \zeta}$, especially for the small values of $m$, as obtained by the proposed closed-form approximation in (4.8a) and (4.8b). Moreover, the small numerical difference between the values of $m_{m, \tilde{\zeta}}$ and $m_{s, \tilde{\zeta}}$ as predicted by the proposed approximation and the ones obtained by the least square fit, and hence $\tilde{\epsilon}$, decreases as $N$ gets large. This can be seen for $m_{m}=m_{s}=2$, in Fig. 4.2, where the approximation improves as $N$ increases. 


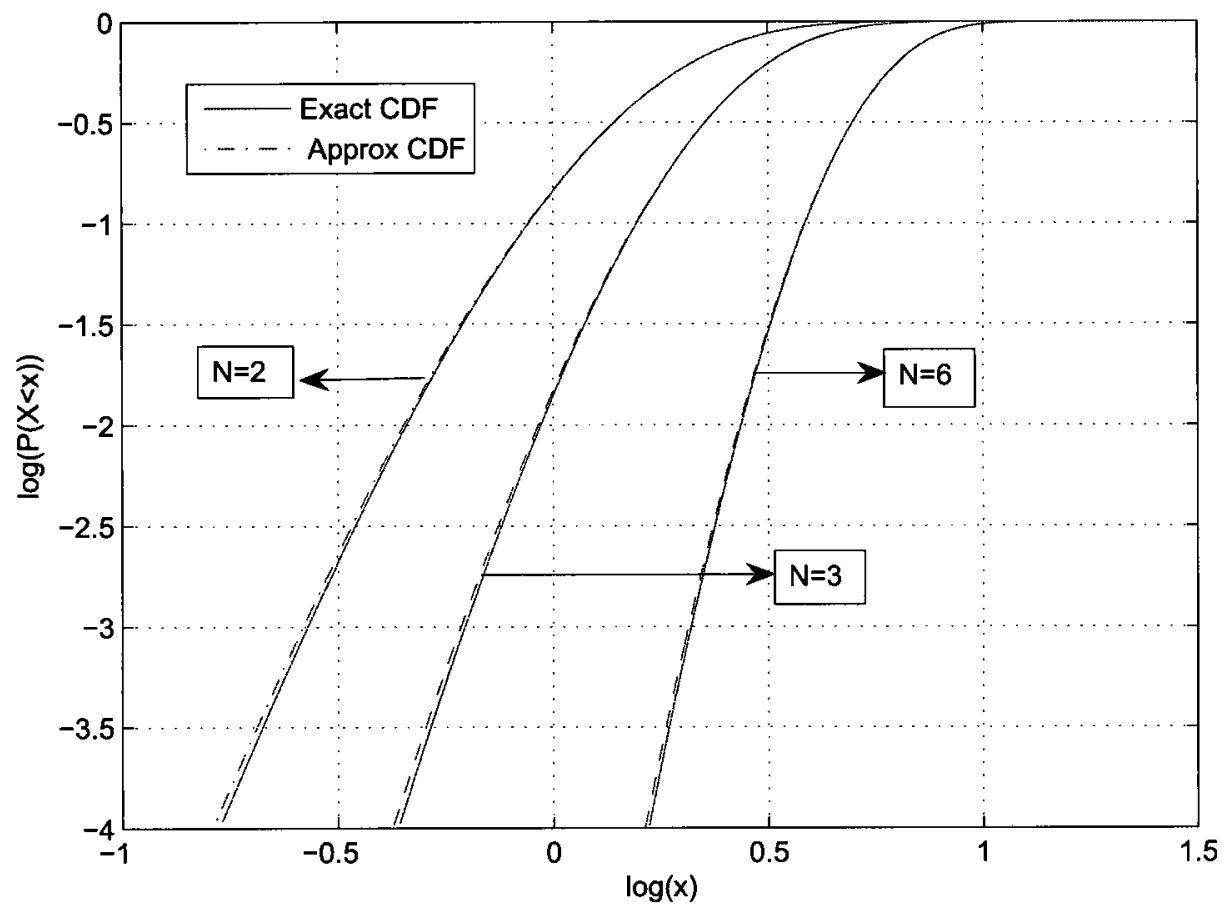

Figure 4.5: The log-log (to base 10) CDF plots for the sum of generalized- $K$ RVs and the approximating generalized- $K$ RV for different values of $N$ with $m_{m}=3$ and $m_{s}=6$.

\subsubsection{Case of i.n.d. Generalized- $K$ RVs}

In the case of the sum of independent and non-identically distributed (i.n.d.) generalized$K$ RVs with arbitrary $m_{m}, m_{s}$, and $\Omega_{0}$ values, we may express the AF of the sum as

$$
\mathrm{AF}_{\text {sum }, \text { i.n.d. }}=\frac{\sum_{j=1}^{N} \mathrm{AF}_{j} \Omega_{0, j}^{2}}{\left(\sum_{j=1}^{N} \Omega_{0, j}\right)^{2}} .
$$

Setting $\mathrm{AF}_{\text {sum,in.d. }}=\mathrm{AF}_{\zeta}$ results in the analogous expression of the one in (4.10) as

$$
\frac{\sum_{j=1}^{N} \mathrm{AF}_{j} \Omega_{0, j}^{2}}{\left(\sum_{j=1}^{N} \Omega_{0, j}\right)^{2}}=\frac{1}{m_{m, \tilde{\zeta}_{i . n . d .}}}+\frac{a_{\tilde{\zeta}_{i, n . d .}}}{m_{m, \tilde{\zeta}_{i . n . d .}}}+\frac{a_{\tilde{\zeta}_{i . n . d .}}}{m_{m, \tilde{\zeta}_{i . n . d .}}^{2}}
$$


which can be solved, for $m_{m, \zeta}$, to give

$$
\begin{gathered}
m_{m, \tilde{\zeta}_{i . n . d .}}=\frac{\left(1+a_{\tilde{\zeta}_{i . n . d}}\right) \Omega_{0, s}^{2}+\Omega_{0, s} \sqrt{\left(1+a_{\tilde{\zeta}_{i . n . d}}\right)^{2} \Omega_{0, s}^{2}+4 a_{\tilde{\zeta}_{i . n . d .}} \sum_{j=1}^{N} \mathrm{AF}_{j} \Omega_{0, j}^{2}}}{\sum_{j=1}^{N} \mathrm{AF}_{j} \Omega_{0, j}^{2}}, \\
m_{s, \tilde{\zeta}_{i . n . d .}}=\frac{m_{m, \tilde{\zeta}_{i . n . d .}}}{a_{\tilde{\zeta}_{i . n . d .}}} \\
\Omega_{0, \tilde{\zeta}_{i . n . d .}}=\sum_{j=1}^{N} \Omega_{0, j}
\end{gathered}
$$

where $\Omega_{0, s}=\sum_{j=1}^{N} \Omega_{0, j}$.

The expression in (4.14a) reduces to the one in (4.8a) for the i.i.d. case. The appropriate value of $a_{\tilde{\zeta}_{i . n . d .}}$ is dependent on the individual ratios $(a \cdot s)$ and can be obtained analytically by matching higher-order moments, which will lead to involved expressions, or can be calculated numerically through the minimization of the difference (for example, the least-squares difference) between the approximated and the approximating PDFs. However, as demonstrated in Fig. 4.6, numerical evaluations indicate that the use of $a_{\tilde{\zeta}_{\text {i.n.d. }}}=1$ leads to a sufficiently accurate approximation when either (i) $m_{m}$ and $m_{s}$ are small and the $a$ ratio of each of the individual generalized$K$ RVs does not allow approximating the generalized- $K$ RV by a Gamma RV (i.e., $1 / 5<a_{i}<5, i=1, \ldots, N$ ) or (ii) the individual ratios, $a$ 's, are too different from each other.

For the i.n.d. case, the CDF plot for the sum of two generalized- $K$ RVs with $m_{m, 1}=2, m_{s, 1}=4, m_{m, 2}=3, m_{s, 2}=1$, and $\Omega_{0,1}=\Omega_{0,2}=1$ is given in Fig. 4.6; it shows the good fit obtained by the proposed selection of $a_{\tilde{\zeta}_{\text {i.n.d. }}}=1$ when the individual ratios, $a$ s, are different. 


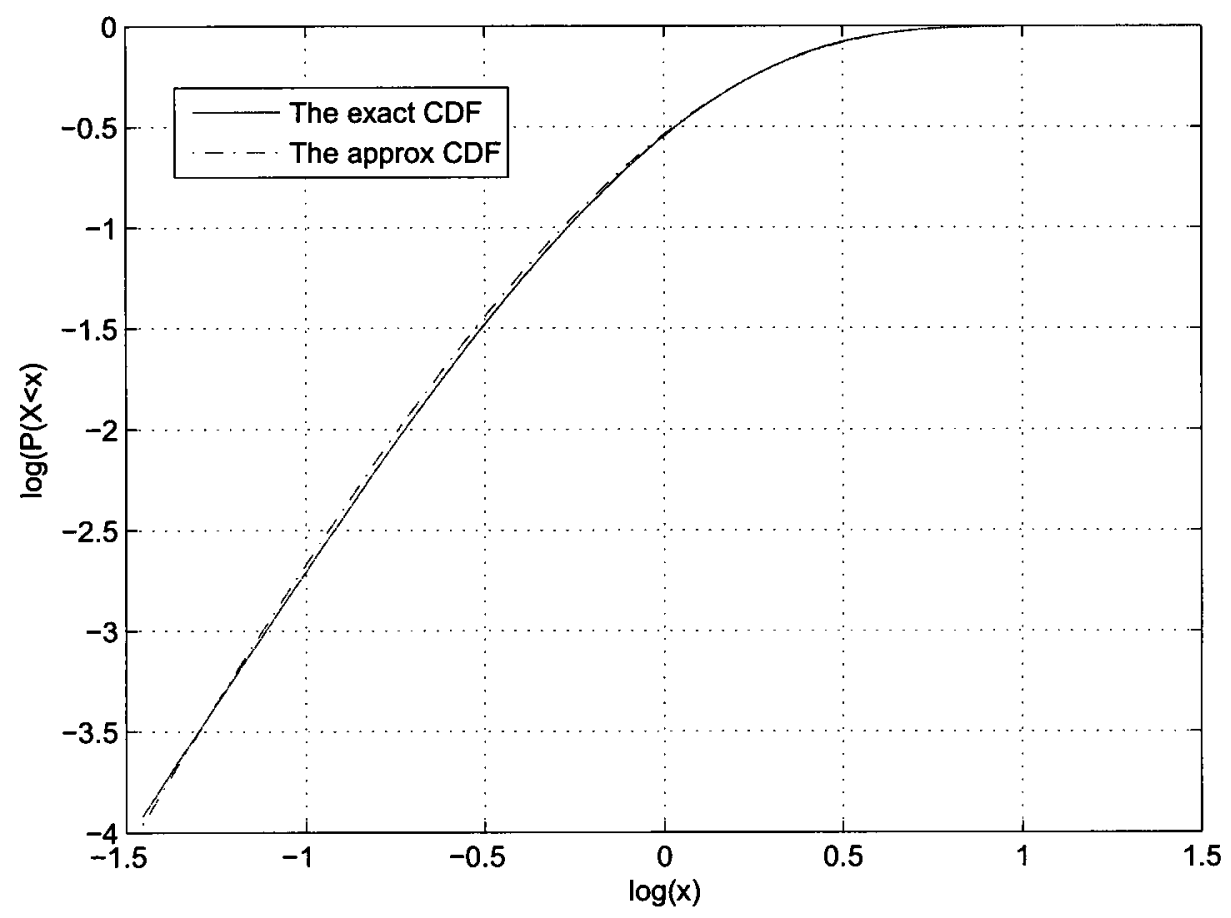

Figure 4.6: The log-log (to base 10) CDF plot for the sum of two generalized- $K$ RVs and the approximating generalized- $K$ RV for $N=2$ with $m_{m, 1}=2, m_{s, 1}=4$, and $m_{m, 2}=3, m_{s, 2}=1$.

\subsection{The Approximate Distribution of the Weighted Sum of Independent Generalized- $K$ RVs}

The proposed moment matching approach used in the previous section can be further used to approximate the PDF of a weighted sum of independent generalized- $K$ RVs that can be expressed as

$$
\zeta_{w}=c_{1} z_{1} w_{1}+c_{2} z_{2} w_{2}+\ldots+c_{N} z_{N} w_{N}
$$

where the weights are assumed to be deterministic and are ordered in a descending order such that $c_{1}>c_{2}>\ldots>c_{N}>0$. 
The AF for the sum in (4.15) can be expressed as

$$
\mathrm{AF}_{\zeta_{w, i . n . d .}}=\frac{\sum_{j=1}^{N} c_{j}^{2} \mathrm{AF}_{j} \Omega_{0, j}^{2}}{\left(\sum_{j=1}^{N} c_{j} \Omega_{0, j}^{2}\right)}
$$

For the i.i.d. case, the expression in (4.16) reduces to

$$
\mathrm{AF}_{\zeta_{w, i . i . d .}}=\frac{\sum_{j=1}^{N} c_{j}^{2} \mathrm{AF}}{\left(\sum_{j=1}^{N} c_{j}\right)^{2}}
$$

Equating the AF in (4.16) to the AF of the approximating generalized- $K$ RV (similar to the expressions in (4.10) will result in

$$
\begin{gathered}
m_{m, \tilde{\zeta_{w}}}=\frac{\left(\left(1+a_{\tilde{\zeta_{w}}}\right)+\sqrt{\left(1+a_{\tilde{\zeta_{w}}}\right)^{2}+\frac{4}{\tilde{c} m_{m} m_{s}} k_{1} a_{\tilde{\zeta}}}\right)}{2\left(1+a+\frac{1}{m_{s}}\right)} \tilde{c} m_{m} \\
m_{s, \tilde{\zeta_{w}}}=\frac{m_{m, \tilde{\zeta_{w}}}}{a_{\tilde{\zeta w}}}, \\
\Omega_{0, \tilde{\zeta_{w}}}=\sum_{j=1}^{N} \Omega_{0, j}
\end{gathered}
$$

where $\tilde{c}=\frac{\left(\sum_{j=1}^{N} c_{i j}\right)^{2}}{\sum_{j=1}^{N} c_{i j}^{2}}$.

The parameter $\tilde{c}$ is always greater than unity since the sum of squares of positive numbers is less than or equal to the square of the sum and is maximum when the weights are equal. So, the resulting parameters of the approximating generalized- $K$ PDF decrease as the disparity between the weights increases and tend to underestimate the values of the multipath fading and shadowing parameters that of the best-fitting generalized- $K \mathrm{PDF}$ in the lower tail region. For these cases, the in- 


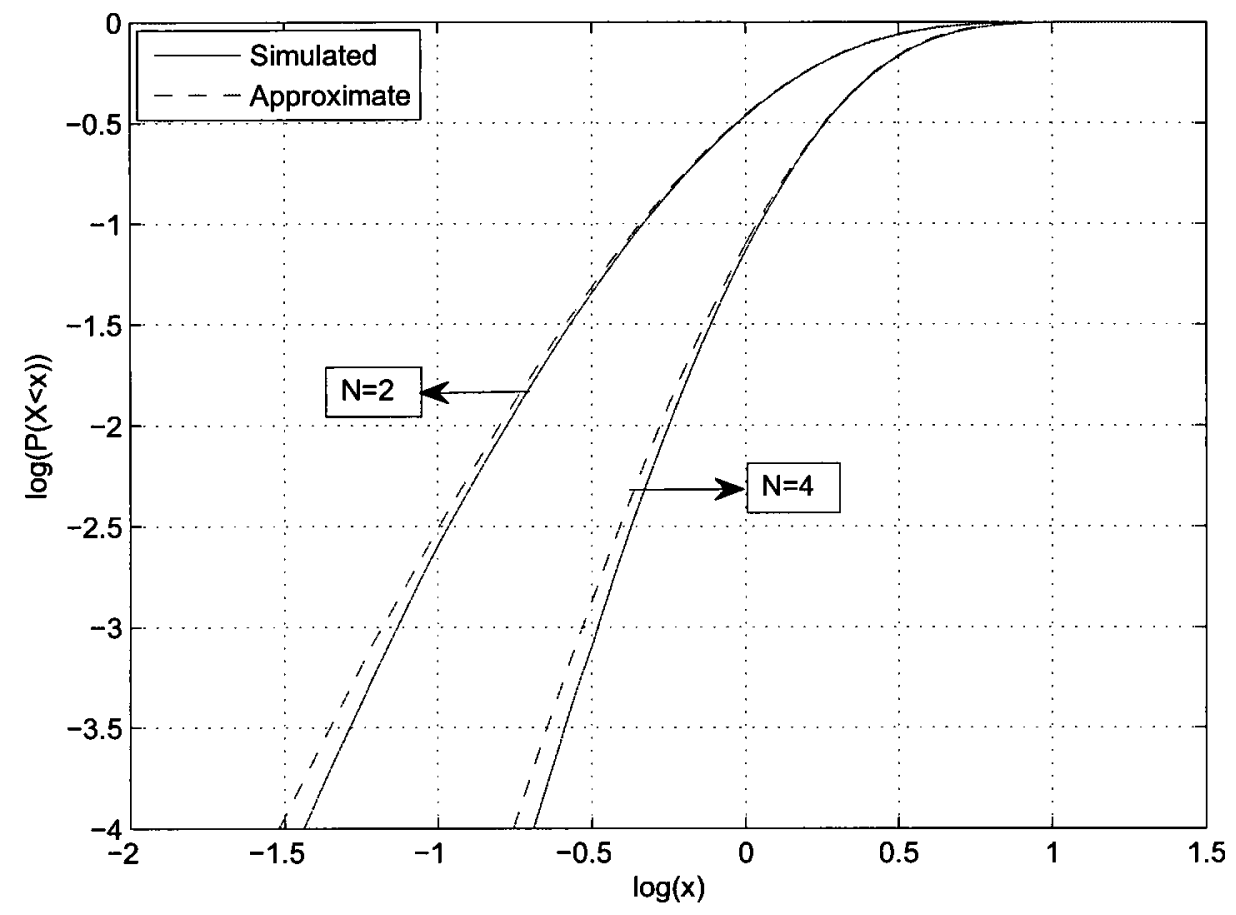

Figure 4.7: The log-log (to base 10) CDF plots for the weighted sum of generalized- $K$ RVs and the approximating generalized- $K$ RV for different values of $N$ with $m_{m}=$ $m_{s}=2$ and $c_{1}=1, c_{2}=0.75, c_{3}=0.5$, and $c_{4}=0.5$.

troduced approximation represents a lower bound and the approximation accuracy can be enhanced by an adjustment parameter $\epsilon_{w}$ (i.e., $\tilde{m}_{m, \tilde{\zeta_{w}}}=m_{m, \tilde{\zeta_{w}}}+\epsilon_{m, w}$ and $\left.\tilde{m}_{s, \tilde{\zeta_{w}}}=m_{s, \tilde{\zeta_{w}}}+\epsilon_{s, w}\right)$ that can be determined numerically. The CDF plots are shown in Figs. 4.7 and 4.8 for $m_{m}=m_{s}=2$ and $m_{m}=m_{s}=4$ (such values of $m_{m}$ and $m_{s}$ are expected since the fading severity gets less for reduced access distances). The plots demonstrate that the approximation accuracy improves as the values of $m_{m}$ and $m_{s}$ increases.

\subsection{On the Statistics of the Sum of Correlated Generalized- $K$ RVs}

The distribution of the sum of correlated generalized- $K \mathrm{RVs}$ is essential for the performance analysis of diversity schemes since the shadowing components are expected 


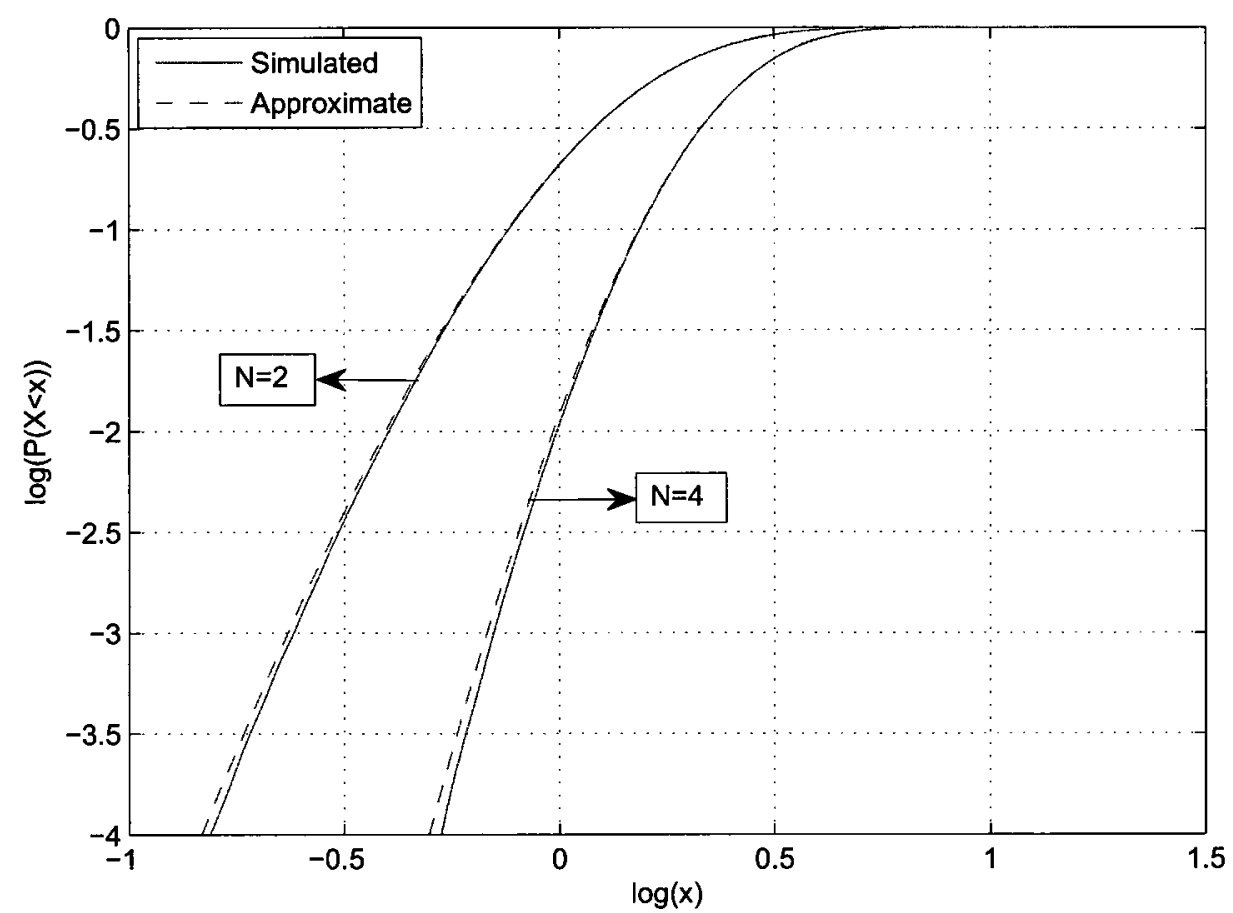

Figure 4.8: The $\log -\log$ (to base 10) CDF plots for the weighted sum of generalized- $K$ RVs and the approximating generalized- $K$ RV for different values of $N$ with $m_{m}=$ $m_{s}=4$, and $c_{1}=1, c_{2}=0.75, c_{3}=0.5$, and $c_{4}=0.5$.

to be correlated across the geographically distributed ports in DASs, base stations (BSs) in a multi-cell processing cellular system, or cooperating nodes in a multi-hop network. However, the derivation of the exact sum distribution has shown to be involved even for i.i.d. case [52], and no results have been reported so far for the correlated case (one exception is the simple special case mentioned in [71] where the shadowing components are assumed to be fully correlated) and the bivariate case in [65]. This motivates, as an alternative, deriving the moments of the sum of correlated generalized- $K$ RVs and utilizing them in moment-based performance analysis. 


\subsubsection{The AF for the Sum of Identically Distributed Corre- lated Generalized- $K$ RVs}

The sum of $N$ correlated generalized- $K$ RVs can be written, using the fact that each generalized- $K \mathrm{RV}$ is the product of two independent Gamma RVs, as

$$
\xi=z_{1} w_{1}+z_{2} w_{2}+\ldots+z_{N} w_{N}
$$

where $z_{i}$ and $w_{i}$ denote the multipath fading and shadowing $\mathrm{RVs}$, respectively. In general, while the multipath and shadowing components are independent, correlation among the $z_{i}$ s and correlation among the $w_{i}$ s may exist in certain physical scenarios as discussed before.

In order to derive the AF expression for the sum of correlated generalized- $K$ RVs, first we need to develop the correlation coefficient expression between two generalized$K \mathrm{RVs}$ in terms of the correlation coefficients between their multipath and shadowing components, respectively. The relevance of revealing the individual roles of the multipath correlations and the shadowing correlations is motivated by the fact that these correlations take place at two different spatial scales as discussed before.

Lemma 4.1: The correlation coefficient between two generalized- $K \mathrm{RVs}, \rho_{i, j}$, can be expressed in terms of the correlation coefficient between their multipath components $\left(\rho_{z_{i}, z_{j}}\right)$ and the correlation coefficient between their shadowing components $\left(\rho_{w_{i}, w_{j}}\right)$ as

$$
\rho_{i, j}=\frac{\rho_{z_{i}, z_{j}} \sqrt{m_{s, i} m_{s, j}}+\rho_{w_{i}, w_{j}} \sqrt{m_{m, i} m_{m, j}}+\rho_{z_{i}, z_{j}} \rho_{w_{i} w_{j}}}{\sqrt{\left(m_{m, i}+m_{s, i}+1\right)} \sqrt{\left(m_{m, j}+m_{s, j}+1\right)}}
$$

and this simplifies for the identically distributed (i.d.) case to ${ }^{1}$

$$
\rho_{i, j}=\frac{\rho_{z_{i}, z_{j}} m_{s}+\rho_{w_{i}, w_{j}} m_{m}+\rho_{z_{i}, z_{j}} \rho_{w_{i}, w_{j}}}{m_{m}+m_{s}+1} .
$$

\footnotetext{
${ }^{1}$ The same $\rho_{i, j}$ expression for the i.d. case has recently been reported in [65] using a more involved approach.
} 
Proof: The proof is given in Appendix B.

In the following proposition, based on Lemma 1, the AF for the sum in (4.19) is expressed in terms of the multipath fading and shadowing correlation coefficients.

Proposition 4.2: The AF of the sum of identically distributed and equally correlated (i.d.e.c.) $N$ generalized- $K$ RVs can be expressed as

$$
\mathrm{AF}_{\xi}^{i . d . e . c}=\frac{1-\rho_{m}}{N m_{m}}+\frac{1-\rho_{s}}{N m_{s}}+\frac{1-\rho_{m} \rho_{s}}{N m_{m} m_{s}}+\frac{\rho_{m}}{m_{m}}+\frac{\rho_{s}}{m_{s}}+\frac{\rho_{s} \rho_{m}}{m_{s} m_{m}}
$$

where $\rho_{m}=\rho_{z_{i} z_{j}}$ and $\rho_{s}=\rho_{w_{i} w_{j}}, \forall i, j=1, \ldots, N$.

Proof: The proof is given in Appendix B.

The results in Lemma 4.1 and Proposition 4.2 have several interesting implications. First, the expressions in (4.21) and (4.22) quantify the individual role of the multipath correlation and the shadowing correlation in the overall correlation between the signals experiencing composite fading, and consequently, in the AF expression for the sum of $N$ i.d.e.c. generalized- $K$ RVs. Secondly, the result in (4.22) indicates the existence of a non-vanishing part of $\mathrm{AF}_{\xi}$, with respect to increasing $N$, in correlated composite fading channels. The existence of this term explains why a correlated composite fading channel cannot approach a non-fading channel $(\mathrm{AF}=0)$ as $N \rightarrow \infty$, since $A F_{\xi}$ is always lower bounded as $A F_{\xi} \geq \rho A F_{0}$, where $A F_{0}$ is the $A F$ of each of the individual summands as given in (3.31). Although the expression in (B.6) applies for an arbitrary correlation model, the equal correlation model, which applies for an array of three antennas placed on an equilateral triangle or for closely spaced antennas other than linear arrays [72], is considered here since it results in the largest AF.

The case where $\rho_{s}>0$ and $\rho_{m} \approx 0$ represents a real scenario in both wireless channels and radar scattering when the fast fading components (multipath or speckle) are uncorrelated while the slow fading (shadowing) components are correlated; the 
corresponding expression of the AF is

$$
\mathrm{AF}_{\xi}^{i . d . e . c . ~}=\frac{1}{N m_{m}}+\frac{1-\rho_{s}}{N m_{s}}+\frac{1}{N m_{m} m_{s}}+\frac{\rho_{s}}{m_{s}}
$$

We observe from (4.23) that the AF due to the multipath component will vanish as $N \rightarrow \infty$ and the non-vanishing part (as $N \rightarrow \infty$ ), as expected, is due to the correlated shadowing. However, that non-vanishing part, with respect to increasing $N$, disappears as $m_{s} \rightarrow \infty$ due to the fact that the Gamma PDF, corresponding to the shadowing component, approaches a Dirac Delta PDF as $m_{s} \rightarrow \infty$ so that the resulting composite fading PDF will be mainly the Gamma PDF associated with the power of the multipath fading component.

Remark 4.6: The existence of negative shadowing correlations was reported in some propagation scenarios $[73,74]$. So, it is of relevance to analytically investigate the effect of such correlations. The expression in (4.23) shows that negative shadowing correlation tends to decrease the non-vanishing term and to increase the part of the AF that vanishes as $N \rightarrow \infty$. The gain due to the existence of negative correlations was reported in [74].

For $N=2$, the AF with negative shadowing correlations becomes

$$
\mathrm{AF}_{\xi}^{i . d . e . c}=\frac{1}{2 m_{m}}+\frac{1-\rho_{s}}{2 m_{s}}+\frac{1}{2 m_{m} m_{s}}+\frac{\rho_{s}}{m_{s}} .
$$

The above expression shows that the effect of shadowing disappears for $\rho_{s}=-1$ (where the second and last terms sum to zero). For $N=3$, the positive semi-definiteness (PSD) condition for a $3 \times 3$ correlation matrix can be expressed, using the fact that a necessary and sufficient condition for a symmetric $N \times N$ matrix, $A$, to be PSD is that all the possible principal minors of $A$ are non-negative [75], as $1+2\left(\rho_{1,2} \rho_{1,3} \rho_{2,3}\right)-\left|\rho_{1,2}\right|^{2}-\left|\rho_{1,3}\right|^{2}-\left|\rho_{2,3}\right|^{2} \geq 0$. Using this inequality, the maximum possible value of $\rho_{s}$ is $-1 / 2$ which will also result in canceling the effect of shadowing. 
For $N=4$, it can be found that the corresponding value is $-1 / 3$. In general, it can be proved (using the result in [76, problem 6.2.14(b)]) (see Appendix B) that the maximum allowable value, due to the PSD constraint, is $\rho_{s, \max }=-1 /(N-1), N>1$; hence $\rho_{s, \max }$ approaches zero as $N \rightarrow \infty$. Physically, this takes place since the AF cannot be less than zero.

Note: In the expressions above, the correlation coefficients among the multipath components and the correlation coefficients among the shadowing components are not related to the multipath fading and shadowing parameters, respectively. This assumption is valid for shadowing correlations where the correlation coefficient is dependent on the distance between the receiving antennas and possibly on the angle of arrival [77] and not related to the standard deviation ( $m_{s}$ parameter) [78]. The multipath fading components, for geographically distributed antenna ports/nodes, are typically uncorrelated.

\subsubsection{The AF for the Sum of Non-identically Distributed Cor- related Generalized- $K$ RVs}

A general expression of the AF for non-identically distributed correlated generalized$K$ RVs with arbitrary different values of $m_{m}$ and $m_{s}$ can be obtained by substituting (4.20) in (B.6). However, assuming a uniform power profile (i.e., $\Omega_{0,1}=\Omega_{0,2}=\ldots .=$ $\left.\Omega_{0, N}=\Omega_{0}\right)$, the AF expression becomes

$$
\mathrm{AF}_{\xi}=\frac{\sum_{i=1}^{N} \frac{\left(m_{m, i}+m_{s, i}+1\right)}{m_{m, i} m_{s, i}}+\sum_{i=1}^{N} \sum_{j=1, i \neq j}^{N}\left[\frac{\rho_{w_{i}, w_{j}}}{\sqrt{m_{s, i} m_{s, j}}}+\frac{\rho_{z_{i}, z_{j}}}{\sqrt{m_{m, i} m_{m, j}}}+\frac{\rho_{w_{i}, w_{j}} \rho_{z_{i}, z_{j}}}{\sqrt{m_{m, i} m_{m, j}} \sqrt{m_{s, i} m_{s, j}}}\right]}{N^{2}} .
$$

Furthermore, when only shadowing correlations are present, (4.25) reduces to

$$
\mathrm{AF}_{\xi}=\frac{\sum_{i=1}^{N} \frac{\left(m_{m, i}+m_{s, i}+1\right)}{m_{m, i} m_{s, i}}+\sum_{i=1}^{N} \sum_{j=1, i \neq j}^{N} \frac{\rho_{w_{i}, w_{j}}}{\sqrt{m_{s, i} m_{s, j}}}}{N^{2}}
$$




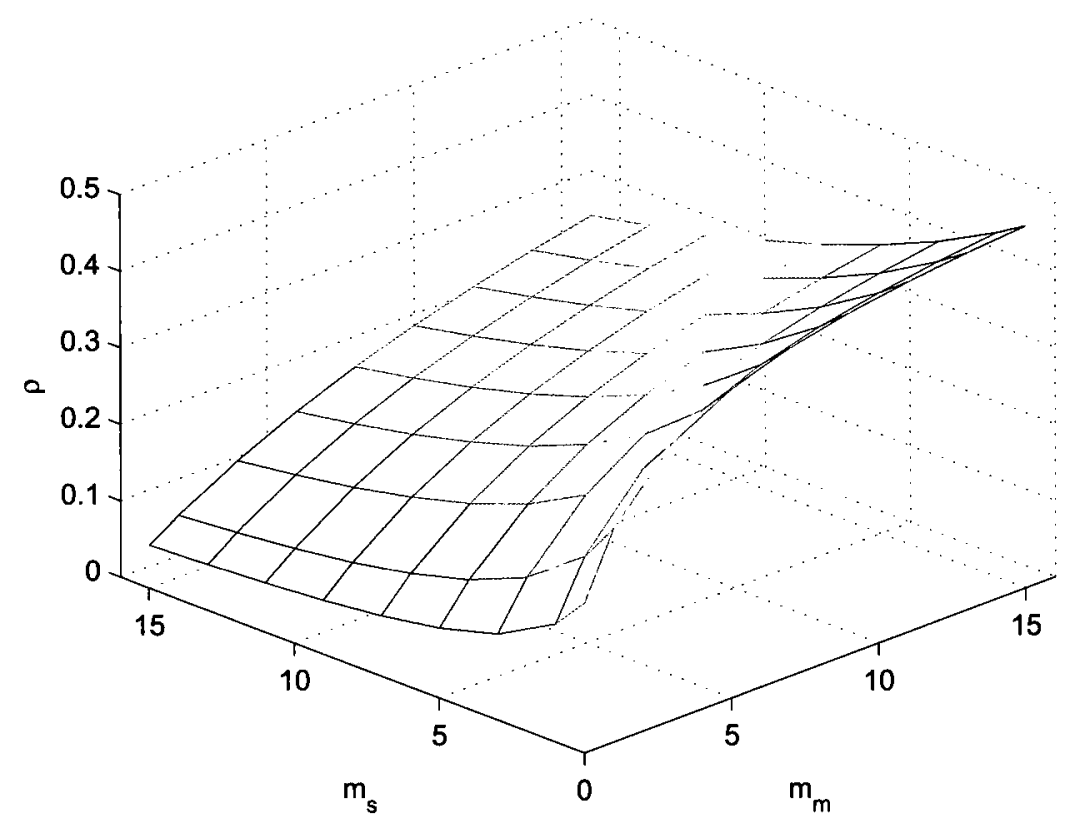

Figure 4.9: The plot for the correlation coefficient between two i.d. generalized- $K$ RVs as a function of their multipath and shadowing parameters.

The plot of the correlation coefficient $\rho_{i, j}$, as given in $(4.21)$, for $\rho_{m}=0, \rho_{s}=0.5$, and for different values of $m_{m}$ and $m_{s}$ is shown in Fig. 4.9. The main observation is that the magnitude of $\rho_{i, j}$ is dependent on the ratio $\frac{m_{s}}{m_{m}}$ which can be seen by re-writing the expression in $(4.21)$, for $\rho_{z_{i}, z_{j}}=0$, as $\rho_{i, j}=\frac{1}{1+\frac{m_{s}}{m_{m}}+\frac{1}{m_{m}}} \rho_{w_{i}, w_{j}}$. This indicates that $\rho_{i, j}$ will decrease as the access distance (between the user terminal and the distributed port) decreases when $m_{s}$ increases at a higher rate in comparison to $m_{m}$, and vice versa.

Using similar derivations, the AF for the sum of weighted and non-identically distributed correlated generalized- $K$ RVs

$$
\mathrm{AF}_{\xi_{w}}=\frac{\sum_{i=1}^{N} c_{i}^{2} \mathrm{AF}_{i} \Omega_{0, i}^{2}+\sum_{i=1}^{N} \sum_{j=1, i \neq j}^{N} \rho_{i, j} \sqrt{\mathrm{AF}_{i}} \sqrt{\mathrm{AF}_{j}} c_{i} \Omega_{0, i} c_{j} \Omega_{0, j}}{\left(\sum_{i=1}^{N} c_{i} \Omega_{0, i}\right)^{2}}
$$


where $\xi_{w}$ is the weighted form of the RV in (4.19).

For the i.i.d. case, the AF reduces to

$$
\mathrm{AF}_{\xi_{w}}=\frac{\left[\sum_{i=1}^{N} c_{i}^{2}+\sum_{i=1}^{N} \sum_{j=1, i \neq j}^{N} \rho_{i, j} c_{i} c_{j}\right] \mathrm{AF}}{\left(\sum_{i=1}^{N} c_{i}\right)^{2}} .
$$

As expected, the expression in (4.28) further reduces to the one in (4.22) for $c_{1}=$ $c_{2}=\ldots=c_{N}$.

Now, using the ergodic capacity approximate expression of a maximal ratio combining receiver given in terms of the diversity factor (DF), which is simply the reciprocal of the AF, as in [79, Eqn. 3], we may write

$$
C \approx \log _{2}(1+\mathrm{SNR})-\frac{\log _{2} e}{2}\left[\frac{1}{N m_{m}}+\frac{1-\rho_{s}}{N m_{s}}+\frac{1}{N m_{m} m_{s}}+\frac{\rho_{s}}{m_{s}}\right]\left(\frac{\mathrm{SNR}}{1+\mathrm{SNR}}\right)^{2}
$$

Now, as $N$ and $m_{m}$ increase, the AF decreases (the DF increases) and hence the approximation in (4.29) tightens and reduces to

$$
C \approx \log _{2}(1+\mathrm{SNR})-\frac{\log _{2} e}{2} \frac{\rho_{s}}{m_{s}}\left(\frac{\mathrm{SNR}}{1+\mathrm{SNR}}\right)^{2}, \rho_{s} \geq 0
$$

Since the approximation is tight for $\mathrm{DF}>2$ (i.e., $\mathrm{AF}<0.5$ ), then $\frac{\rho_{s}}{m_{s}}<0.5$ (according to (4.26)). For a DAS with correlated shadowing components, we may set $\rho_{s} \approx 0.5$, leading to $m_{s}>1$ which corresponds to $\sigma_{s}<7.2 \mathrm{~dB}$; such non-severe shadowing levels are expected in urban areas where DASs are typically deployed.

The ergodic capacity loss with respect to AF and SNR is shown in Fig. 4.10. The main observation is that as both $N \rightarrow \infty$ and SNR $\rightarrow \infty$, the loss approaches a limiting value (power offset) that can be easily quantified, using (4.30), or [79, Eqn. 9], as $L_{\infty}=\frac{\log _{2} e}{2} \frac{\rho_{s}}{m_{s}}, \rho_{s} \geq 0$. Using the plot in Fig. 4.10, the loss in ergodic capacity can be predicted for different values of $\rho_{s}$ and $m_{s}$ for $\frac{\rho_{s}}{m_{s}}<0.5$. 


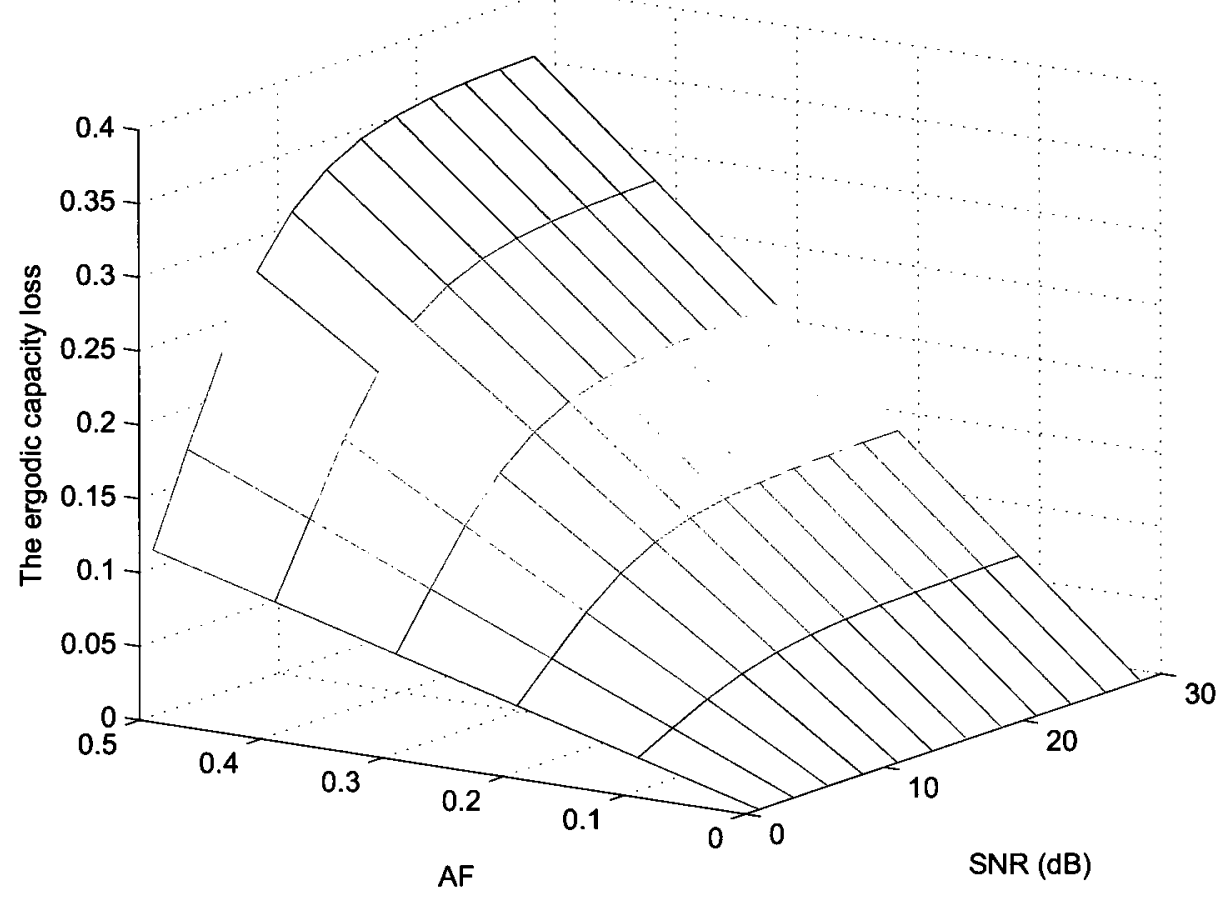

Figure 4.10: The plot for the ergodic capacity loss versus the AF and the SNR.

Mathematically, the expression in (4.30) is valid only for the positive values of $\rho_{s}$ due to the PSD constraint as explained before; physically, the capacity of a fading channel, without channel state information at the transmitter, can not exceed the capacity of the corresponding additive white Gaussian noise (AWGN) channel.

\subsection{Conclusions}

In this chapter, the statistics for the sum and the weighted sum of generalized- $K$ RVs are investigated. First, the adjustable moment matching method, as introduced in Chapter 3, is utilized to approximate, region-wise, the PDF of the sum of i.i.d. generalized- $K$ RVs by a Gamma PDF. Next, the approximation of the sum distribution by another generalized- $K$ distribution is introduced and is extended to the weighted sum of independent generalized- $K$ RVs. Finally, the expressions of the AF for the sum and the weighted sum of correlated generalized- $K$ RVs are developed. 
The approximate distributions for the weighted sum are further utilized in Chapter 6 for deriving the corresponding expressions for the ergodic capacity and information outage probability for DASs in cellular networks. On the other hand, the AF for the sum of correlated generalized- $K$ RVs can be utilized further in moment-based analysis of MRC diversity scheme in generalized- $K$ channels with correlated shadowing components. 


\section{Chapter 5}

\section{On the Capacity of MIMO Channels with Common and Individual Power Constraint}

In DASs, the individual power constraints can be imposed on each transmit antenna port since each of them has its RF chain. This motivates looking into the capacity of MIMO channels with such constraints. Moreover, in engineering design, we are often interested in not only the numerical solution of a problem, but also in the details of the structure of the optimal solution. This chapter presents a direct approach for the maximization of mutual information in MIMO channels using the standard Lagrangian formulation to investigate the range of the optimality of reduced-rank signaling in MIMO channels with individual power constraint. Although the capacity of point-to-point MIMO channels with a common power constraint was characterized, for deterministic channel matrix or perfect channel state information (CSI) (for fading channels), using the singular value decomposition (SVD) approach which has led to a numerically efficient tool to compute the channel capacity; the SVD approach basically transforms the physical channels into a virtual ones where the gain of each channel is quantified by the corresponding singular value of the channel matrix. Subsequent analysis of the capacity of MIMO channels were based on studying the statistics of these gains (eigenmodes) for both uncorrelated and correlated scenarios. In this chapter, the Lagrangian method is utilized to characterize the optimal 
input covariance matrix for TIMO (two-input multiple-output) channels for both the common and individual power constraint cases. The obtained results are applicable for up-link transmission in single-user schemes where the user terminal is equipped by a two transmit antennas and the CSI feedback is limited.

\subsection{Related Work}

The literature on MIMO channels is vast since this topic has been a very active one over the last decade. In here, the main contributions on the characterization of the optimal input covariance matrix in MIMO channels are highlighted. The informationtheoretic characterization of MIMO channels was launched by the seminal papers $[80,81]$ where the main finding was that the capacity of a MIMO channel, for rich scattering environments, increases linearly proportional to the minimum number of transmit and receive antennas. In [81], it was shown that if the channel realization is known at both the receiver and the transmitter, then the "water-filling" algorithm [82] can be applied after converting the MIMO channel into a set of parallel channels using the singular value decomposition of the channel matrix. It was also shown that when only the channel distribution is known at the transmitter, the optimal "capacity-achieving" transmission scheme for i.i.d. Rayleigh channels is the isotropic Gaussian input. Subsequent work has been on the characterization of the MIMO channel capacity under more realistic assumptions on the channel model and the availability of the channel state information at both the transmitter and the receiver.

The effect of channel correlations on the capacity of MIMO systems was considered in [83] where it was shown that fading correlation affects the MIMO capacity by modifying the distributions of the channel eigenmodes and the term "effective degrees of freedom" was introduced to quantify that effect. In [84], it was shown that the linear growth rate of capacity in correlated MIMO channels is smaller than the 
uncorrelated case. The analytical tool used in [84] relies on the Stieltjes transform of the asymptotic eigenvalue distribution. Another line of research has considered the fact that even partial CSI such as the channel covariance information or the channel mean information at the transmitters (CSIT) helps increase capacity [85]. In [86], it was shown that with multiple receive antennas and imperfect feedback, beamforming becomes the optimal strategy as the eigenvalues of the channel covariance matrix become more disparate (due to strong correlations among the columns of the channel matrix), as the variance of noise increases, and/or as the transmit power decreases. This result was extended to account for receive correlations in [87]. A review of the existing literature on the capacity-achieving input covariance matrix is presented in [88].

In the literature on the design of the optimal transmission scheme for Gaussian multiple-input multiple-output (MIMO) channels, $[86,87]$ and references therein, the range of optimality of beamforming is of relevance since scalar coding can be used to achieve the channel capacity. Moreover, the introduction of distributed MIMO systems [16] and MIMO-OFDM (orthogonal frequency-division multiplexing) systems [89] has motivated the research on the optimal input covariance for MIMO channels with individual power constraints.

Notation: In this chapter, uppercase letters denote deterministic matrices and bold-faced uppercase letters denote random matrices. For vectors, bold-faced lowercase letters are used for both deterministic and random vectors where the distinction is assumed to be clear context-wise. The determinant, trace, adjoint and Hermitian of a matrix $A$ are $\operatorname{denoted}$ as $\operatorname{det}[A], \operatorname{tr}(A), \operatorname{Adj}(A)$ and $A^{H}$, respectively; and $A \succeq 0$ denotes a positive semi-definite matrix. For a complex number $z$, the conjugate of $z$ and the real and imaginary parts of $z$ are denoted by $z^{*}, \Re(z)$, and $\Im(z)$, respectively. 


\subsection{Capacity of MIMO Channels with a Common Power Constraint}

\subsubsection{Deterministic Channel Matrix}

The discrete-time received signal, at the $i$ th time instant, in a MIMO system with $L$ transmit and $M$ receive elements, as shown in Fig. 5.1, can be expressed as

$$
\mathbf{y}(i)=H \mathbf{x}(i)+\mathbf{n}(i)
$$

where $\mathbf{x}$ is the transmitted vector and $\mathbf{n}$ denotes the complex Gaussian noise vector

$$
\begin{gathered}
H=\left[\begin{array}{cccc}
h_{1,1} & h_{1,2} & \ldots & h_{1, L} \\
h_{2,1} & h_{2,2} & \ldots & h_{2, L} \\
\vdots & \vdots & \vdots & \vdots \\
h_{M, 1} & h_{M, 2} & \ldots & h_{M, L}
\end{array}\right]=\left[\mathbf{h}_{1}, \ldots \ldots \mathbf{h}_{L}\right] \\
\mathbf{x} \\
=\left[x_{1}, \ldots x_{L}\right] \\
\mathbf{n}=\left[n_{1} \ldots \ldots n_{M}\right] .
\end{gathered}
$$

In (5.1), the $M \times L$ channel matrix $H$ is deterministic and the noise components for all receive elements are identical and independent zero-mean complex Gaussian RVs with variance $\sigma_{n}^{2}$.

The channel capacity of a MIMO channel for a deterministic channel matrix $H \in$ $\mathcal{C}^{M \times L}$ can be expressed as $[81]^{1}$

$$
C=\max _{Q \succeq 0, \operatorname{tr}(Q) \leq P} \log \operatorname{det}\left[I_{M}+\frac{1}{\sigma_{n}^{2}} H Q H^{H}\right]=\max _{Q \succeq 0, \operatorname{tr}(Q) \leq P} \operatorname{logdet}\left[I_{L}+\frac{1}{\sigma_{n}^{2}} Q H^{H} H\right]
$$

\footnotetext{
${ }^{1}$ In this chapter, the $\log$ is to base 2 unless otherwise specified.
} 


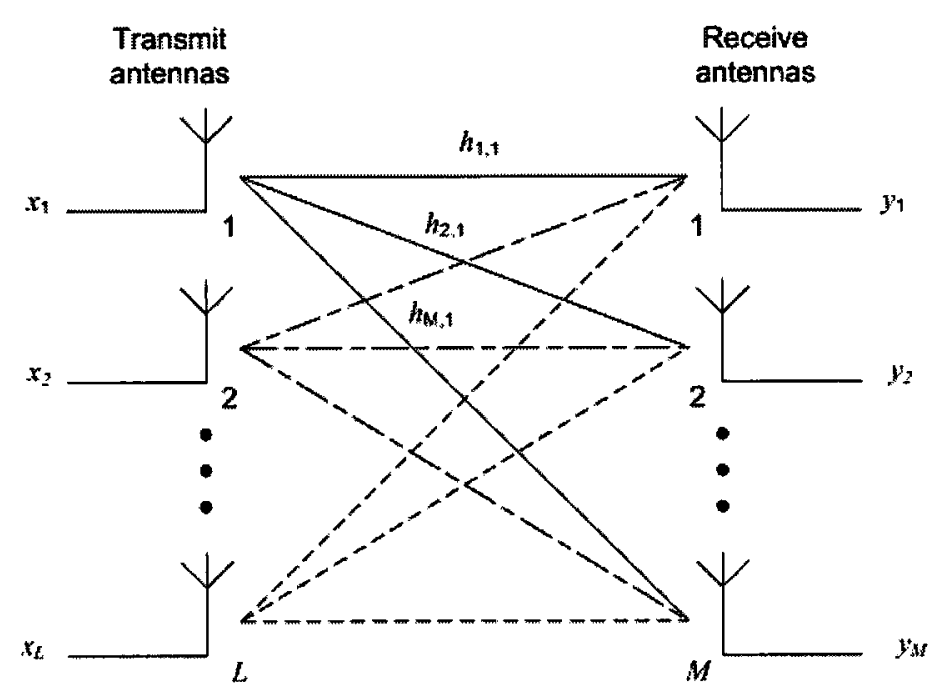

Figure 5.1: A MIMO channel with $L$ transmit and $M$ receive antennas.

where $P$ is the total power constraint and $Q \in \mathcal{C}^{L \times L}$ is the positive semi-definite covariance matrix of the proper complex Gaussian input vector [90]. In (5.3), $I_{M}$ denotes the $M \times M$ identity matrix and $\sigma_{n}^{2}$ is the variance of the complex Gaussian noise at each receive element. In subsequent derivations $\sigma_{n}^{2}$ is set to unity for notational simplicity.

\section{The Singular Value Decomposition (SVD) Approach}

The singular value decomposition (SVD) of the channel matrix results in $H=U \Lambda V$ where $U$ and $V$ are unitary, and $\Lambda$ is a diagonal matrix whose entries are the singular values of $H$ or square roots of the eigenvalues of $H^{H} H$ (denoted as $\lambda$ ). The columns of $U$ are the eigenvectors of $H^{H} H$ and the columns of $V$ are the eigenvectors of $H^{H} H$. In the SVD approach, this decomposition is used to transform the physical MIMO channel into a set of parallel (orthogonal) channels having gains that are proportional to the non-zero singular values of the channel matrix so that the original channel is 
equivalent to the following channel

$$
\tilde{\mathbf{y}}=\Lambda \tilde{\mathbf{x}}+\tilde{\mathbf{n}}
$$

where $\tilde{\mathbf{y}}=U^{H} \mathbf{y}, \tilde{\mathbf{x}}=V^{H} \mathbf{x}$ and $\tilde{\mathbf{n}}=U^{H} \mathbf{n}$. Since the rank $H$ is equal to the number of its non-zero singular values and is at most $\min (M, L)$, then $(5.4)$ can be written component-wise as

$$
\tilde{y}=\sqrt{\lambda_{i}} \tilde{x}+\tilde{n}, i=1, \ldots, \min (M, L)
$$

The mutual information is maximized by choosing the inputs to be independent zero-mean complex Gaussian variables whose variances are determined via the power allocation scheme known as "water-filling" as

$$
E\left[\Re(\tilde{x})^{2}\right]=E\left[\Im(\tilde{x})^{2}\right]=\frac{1}{2}\left[\nu-\lambda^{-1}\right]^{+}
$$

where $\nu$ is chosen to meet the input power constraint, $[x]^{+}$denotes $\max (0, x)$ and $E$ is the expectation operator. The resulting capacity is given as

$$
C=\sum_{i}\left(\log _{2}[\nu \lambda]\right)
$$

Another interpretation of this approach is that if we decompose the input covariance matrix as

$$
Q=S \Pi S^{H}
$$

where $\Pi$ is a non-negative diagonal matrix and $S$ is a unitary matrix. Then, the columns of $S$ must equal the eigenvectors of the inner product matrix $H^{H} H$ whereas the transmit powers, entries of $\Pi$ corresponding to each eigenvector, can be found via water-filling on the eigenvalues of $H^{H} H$ [81]. 


\subsubsection{Fading Channel Matrix}

In a fading environment, the channel matrix is random and hence the instantaneous mutual information ${ }^{2}$

$$
I(\mathbf{H})=\log \operatorname{det}\left[I_{M}+\frac{1}{\sigma_{n}^{2}} \mathbf{H} Q \mathbf{H}^{H}\right]
$$

is random and the channel capacity depends on the availability of CSI at the transmitter and the receiver, and the channel statistics. If the CSI is available at the transmitter and the receiver, then a well-known result for a fading MIMO channel matrix is that the capacity with perfect CSI at the transmitter (CSIT) and at the receiver (CSIR) is the average of capacities achieved for each "deterministic" channel realization [85]

$$
C=\max _{Q, \operatorname{tr}(Q) \leq P} \log \operatorname{det}\left[I_{M}+\frac{1}{\sigma_{n}^{2}} H Q H^{H}\right]=\max _{Q \succeq 0, \operatorname{tr}(Q) \leq P} \log \operatorname{det}\left[I_{L}+\frac{1}{\sigma_{n}^{2}} Q H^{H} H\right] .
$$

However, if only perfect CSIR is assumed, the ergodic capacity of a MIMO stationary and ergodic channel with perfect CSIR is defined as

$C=\max _{Q, \operatorname{tr}(Q) \leq P} E_{\mathbf{H}} \operatorname{logdet}\left[I_{M}+\frac{1}{\sigma_{n}^{2}} \mathbf{H} Q \mathbf{H}^{H}\right]=\max _{Q \succeq 0, \operatorname{tr}(Q) \leq P} E_{\mathbf{H}} \operatorname{logdet}\left[I_{L}+\frac{1}{\sigma_{n}^{2}} Q \mathbf{H}^{H} \mathbf{H}\right]$,

where the expectation is over the random channel matrix $\mathbf{H}$. However, the ergodicity assumption is not always satisfied, for example, when the channel realization is chosen randomly and remains fixed for the duration of transmission. In this case, the average mutual information does not correspond to an achievable rate since there is always a non-zero probability that the channel realization would not support any positive rate $\mathrm{R}$, so the rate of reliable transmission (the Shannon-sense capacity) is zero and the notion of outage is introduced $[1,85]$.

\footnotetext{
${ }^{2}$ In here, the bold-faced matrix denotes being a random matrix, while for vectors, bold-faced lowercase letters are used for both deterministic and random vectors where the distinction is assumed to be clear context-wise.
} 
Definition 5.1: For a fixed transmission rate $R$, the information outage probability is defined as

$$
P_{\text {out }}=\operatorname{Pr}(I(\mathbf{H})<R)
$$

Definition 5.2: The outage capacity of a MIMO random and non-ergodic channel is defined as the largest rate such that the information outage probability is less than $\epsilon$, i.e.,

$$
C_{\epsilon}=\sup \{R: \operatorname{Pr}(I(\mathbf{H})<R) \leq \epsilon\}
$$

\subsubsection{TIMO Channels with a Common Power Constraint}

For a TIMO channel with a common power constraint, the capacity can be expressed, using (5.3), as

$$
C=\max _{Q, \operatorname{tr}(Q) \leq P} \log \operatorname{det}\left[I_{M}+H Q H^{H}\right]=\max _{Q \succeq 0, \operatorname{tr}(Q) \leq P} \log \operatorname{det}\left[I_{2}+Q H^{H} H\right]
$$

The optimization problem in (5.14) and hence the channel capacity was characterized in [81] by considering the corresponding set of the orthogonal channels, obtained using the SVD approach, and then applying the water-filling power allocation over these channels as described before. However, in here we will consider the solution of (5.14) using standard Lagrangian approach. In this regard, we may express the input covariance matrix of the input complex Gaussian vector as

$$
Q=\left[\begin{array}{cc}
\sigma_{1}^{2} & \rho_{12} \sigma_{1} \sigma_{2} \\
\rho_{21} \sigma_{1} \sigma_{2} & \sigma_{2}^{2}
\end{array}\right] .
$$

It can be shown using the proposed solution in Appendix $C$ that we may express the 
optimal input correlation coefficient as

$$
\rho_{12}=\frac{\mathbf{h}_{1}^{H} \mathbf{h}_{2}}{\sqrt{\frac{P^{2}}{4}-\Delta^{2}}\left[\left|\mathbf{h}_{1}\right|^{2}\left|\mathbf{h}_{2}\right|^{2}-\left|\mathbf{h}_{1}^{H} \mathbf{h}_{2}\right|^{2}\right]+\mu_{1}}, \text { for }\left|\rho_{12}\right|^{2}<1, \Delta \neq \frac{P}{2}, \text { and } \mathbf{h}_{1}^{H} \mathbf{h}_{2} \neq 0
$$

where $\mathbf{h}_{1}$ and $\mathbf{h}_{2}$ denote the first and second columns of the channel matrix, respectively, and $\Delta$ denotes the disparity between the allocated powers as $\sigma_{1}^{2}=\frac{P}{2} \pm \Delta$ and $\sigma_{2}^{2}=\frac{P}{2} \mp \Delta$. Furthermore, the minimum input SNR below which beamforming is optimal is given as

$$
\sqrt{\frac{P^{2}}{4}-\Delta^{2}} \leq \frac{\mathbf{h}_{1}^{H} \mathbf{h}_{2}}{\left[\left|\mathbf{h}_{1}\right|^{2}\left|\mathbf{h}_{2}\right|^{2}-\left|\mathbf{h}_{1}^{H} \mathbf{h}_{2}\right|^{2}\right]}
$$

For MIMO channels with a common power constraint, substituting $\Delta=\Delta_{1}$ as given in (C.7) in Appendix C leads to

$$
P \leq \sqrt{\frac{4\left|\mathbf{h}_{1}^{H} \mathbf{h}_{2}\right|^{2}+\left(\left|\mathbf{h}_{2}\right|^{2}-\left|\mathbf{h}_{1}\right|^{2}\right)^{2}}{\left[\left|\mathbf{h}_{1}\right|^{2}\left|\mathbf{h}_{2}\right|^{2}-\left|\mathbf{h}_{1}^{H} \mathbf{h}_{2}\right|^{2}\right]^{2}}}
$$

The same result can be obtained using the SVD approach since the minimum normalized power can be expressed for the two-input two-output case as $P \leq \frac{1}{\lambda_{2}}-\frac{1}{\lambda_{1}}$ where $\lambda_{1}$ and $\lambda_{2}$ denote the eigenvalues of $H^{H} H$ [91]; this expression can be extended to the TIMO case to get the one in (5.18).

The observation that $\sigma_{1}>0$ and $\sigma_{2}>0$ implies that beamforming takes place in correlated TIMO channels through having $\left|\rho_{12}\right|^{2}=1$ while beamfoming takes place in uncorrelated TIMO channels through having $\left|\rho_{12}\right|^{2}=0$ and $\sigma_{1}=0$ or $\sigma_{2}=0$ (since uncorrelated transmission is always optimal for uncorrelated MIMO channels). Moreover, this explains why the capacity of correlated MIMO channels exceeds the capacity of uncorrelated MIMO channels at low SNR [92]. 


\subsection{Capacity of MIMO Channels with Individual Power Constraints}

In addition to DASs (D-MIMO systems), the problem of determining the capacity of MIMO channels with individual "elemental" power constraints arises in (i) collocated MIMO systems where each antenna is equipped with its own power amplifier, (ii) MIMO-OFDM systems where it is preferable to use uniform power allocation across the transmit antennas, and (iii) digital subscriber lines with individual power constraints per modem.

In general, for a MIMO channel with individual power constraints, the capacity can be expressed as

$$
C=\max _{Q \succeq 0, Q_{i i}=p_{i}} \log \operatorname{det}\left[I_{M}+H Q H^{H}\right]=\max _{Q \succeq 0, Q, Q_{i i}=p_{i}} \log \operatorname{det}\left[I_{2}+Q H^{H} H\right],
$$

where $Q_{i i}$ denotes the $i$ th diagonal element of $Q$ and $\sum_{i=1}^{L} p_{i}=P$.

\subsubsection{TIMO Channels with Individual Power Constraints}

\section{Deterministic Channel Matrix}

For TIMO channels with individual power constraints, the optimization problem is similar to the one in (5.15) but with the diagonal elements of $Q$ being predetermined since the allocated powers are non-zero, hence the optimal input correlation coefficient, analogous to (5.16), can be expressed as

$$
\rho_{12}=\frac{\mathbf{h}_{1}^{H} \mathbf{h}_{2}}{\sqrt{\frac{P^{2}}{4}-\Delta_{2}^{2}}\left[\left|\mathbf{h}_{1}\right|^{2}\left|\mathbf{h}_{2}\right|^{2}-\left|\mathbf{h}_{1}^{H} \mathbf{h}_{2}\right|^{2}\right]+\mu_{2}}, \text { for } 0<\Delta_{2}<\frac{P}{2} \text { and } \mathbf{h}_{1}^{H} \mathbf{h}_{2} \neq 0
$$

where again $\Delta_{2}$ denote the difference in power allocation such that $\sigma_{1}^{2}=\frac{P}{2} \pm \Delta_{2}$, $\sigma_{2}^{2}=\frac{P}{2} \mp \Delta_{2}$, and $\mu_{2}$ has the same expression as that of $\mu_{1}$. 
Proposition 5.1: The range of the normalized input SNR for which rank-1 signaling is optimal (capacity-achieving) for correlated TIMO channels with individual power constraints is given as

$$
0<P \leq 2 \sqrt{\frac{\left|\mathbf{h}_{1}^{H} \mathbf{h}_{2}\right|^{2}}{\left[\left|\mathbf{h}_{1}\right|^{2}\left|\mathbf{h}_{2}\right|^{2}-\left|\mathbf{h}_{1}^{H} \mathbf{h}_{2}\right|^{2}\right]^{2}}+\Delta_{2}^{2}} \text { for } 0<\Delta_{2}<\frac{P}{2} \text { and } \mathbf{h}_{1}^{H} \mathbf{h}_{2} \neq 0
$$

Proof: The proof is straightforwardly obtained using the expression in (5.20) and the fact that $\mu_{2}>0$ for $\left|\rho_{12}\right|^{2}=1$.

The capacity of a MIMO channel with a common power constraint and a MIMO channel with individual power constraints is shown in Fig. 5.1 for $M=2$ and $\Delta=P / 4$. The plots demonstrate the capacity loss due individual power constraints especially for high SNR where equal power allocation is asymptotically optimal. In Fig. 5.2, the capacity of the MIMO channel with individual power constraints is shown along with the achievable rates of uncorrelated transmission (where $\rho_{12}=0$ ) and beamforming. The plots demonstrate the optimality range for beamforming and suggest that proper switching between baemforming and uncorrelated transmission (beyond the beamforming optimality range) can approach the channel capacity.

Remark: For the case $L=3$, the optimization problem can be expanded using the expression in (5.19). However, the condition for $Q$ to be semi-positive definite is more involved for $L=3$. Again using Sylvester's criterion for positive semi-definiteness [75], the non-negativity condition for the principal minors can be expressed as

$$
\begin{gathered}
\sigma_{1}^{2} \geq 0, \sigma_{2}^{2} \geq 0, \sigma_{3}^{2} \geq 0 \\
\left|\rho_{12}\right|^{2} \leq 1,\left|\rho_{13}\right|^{2} \leq 1,\left|\rho_{23}\right|^{2} \leq 1 \\
\sigma_{1}^{2} \sigma_{2}^{2} \sigma_{3}^{2}\left[1+2 \Re\left[\rho_{12} \rho_{23} \rho_{31}\right]-\left|\rho_{12}\right|^{2}-\left|\rho_{31}\right|^{2}-\left|\rho_{23}\right|^{2}\right] \geq 0 .
\end{gathered}
$$

Now, since the allocated powers are non-zero for a MIMO channel with individual 


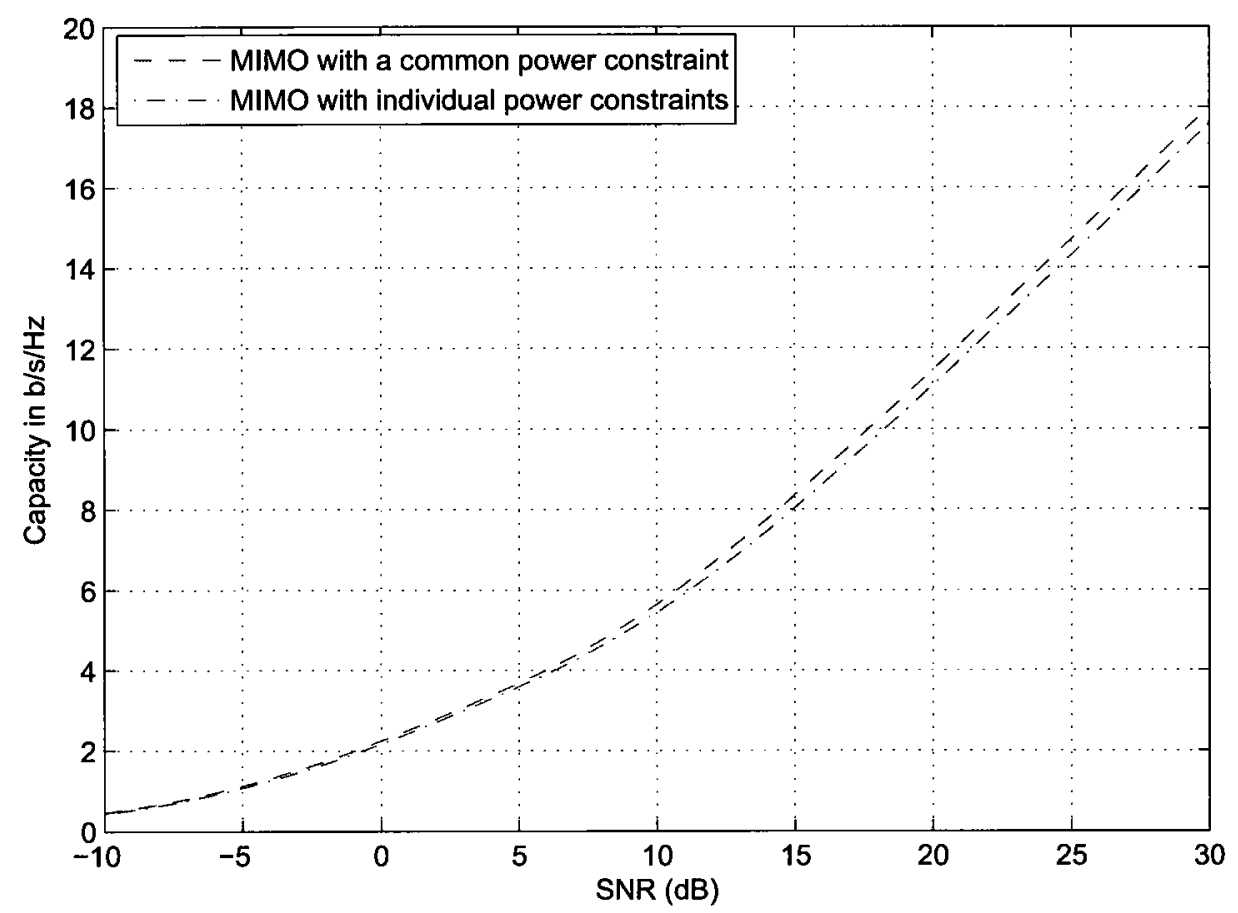

Figure 5.2: The plots of the MIMO capacity with common and individual power constraints.

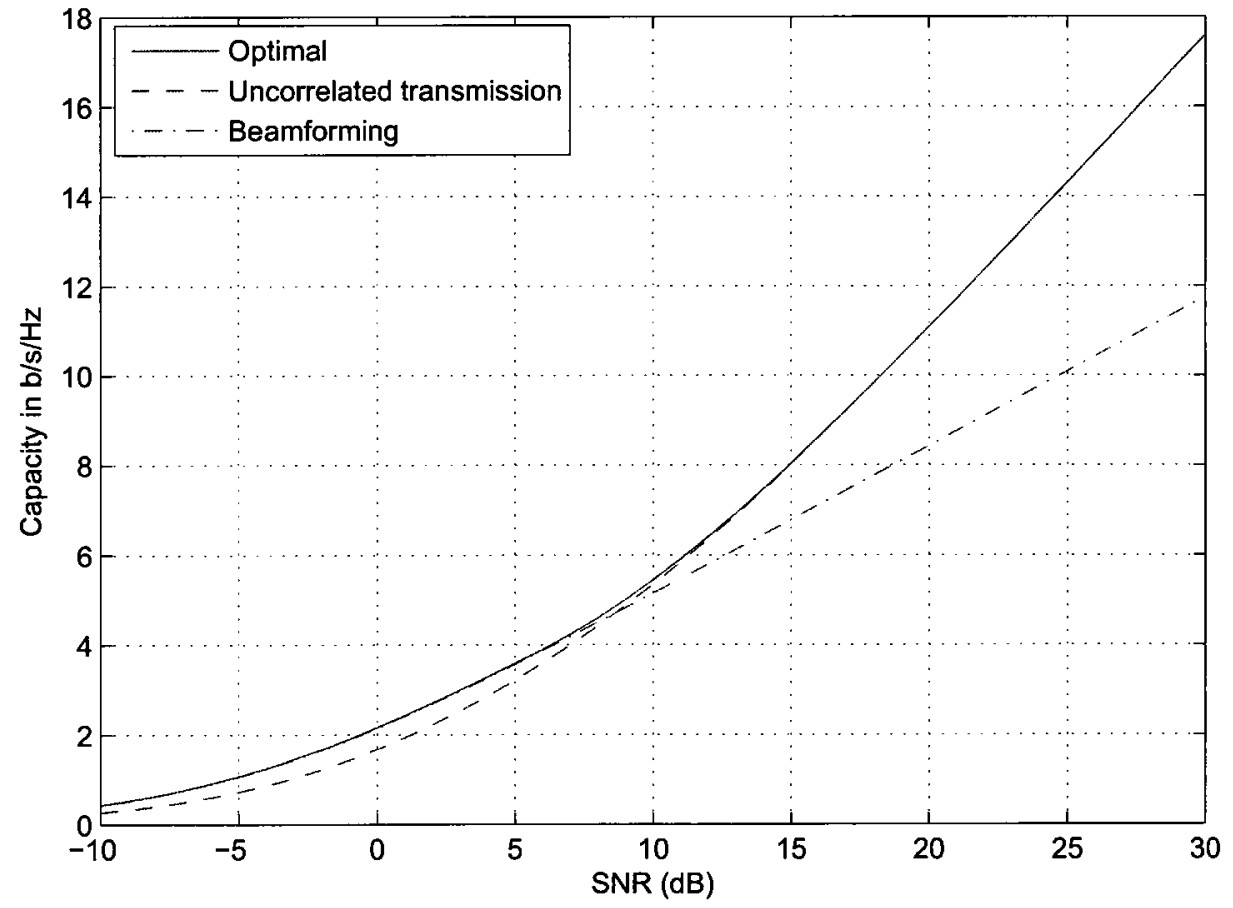

Figure 5.3: The plots of the MIMO capacity with individual power constraints for different transmission schemes. 
power constraints, the problem of finding the rank of $Q$, and hence the Rank-1 case, reduces to computing the corresponding optimal input correlation coefficients and determining the corresponding rank. Using the fact that for the non-singular matrix $I_{L}+Q H^{H} H$

$$
\frac{\partial\left[\operatorname{det}\left(I_{L}+Q H^{H} H\right)\right]}{\partial \rho_{i, j}}=\operatorname{tr}\left(\operatorname{Adj}\left(I_{L}+Q H^{H} H\right) \frac{\partial\left\{I_{L}+Q H^{H} H\right\}}{\partial \rho_{i, j}}\right)
$$

the Karush-Kuhn-Tucker (KKT) conditions needed to solve for the optimal correlation coefficients can be expressed as

$$
\begin{aligned}
& c_{21} \sigma_{1} \sigma_{2}\left|\mathbf{h}_{1}\right|^{2}+c_{22} \sigma_{1} \sigma_{2} \mathbf{h}_{1}^{H} \mathbf{h}_{2}+c_{23} \sigma_{1} \sigma_{2} \mathbf{h}_{1}^{H} \mathbf{h}_{3}+\mu_{2} \rho_{12}+\mu_{3}\left[\rho_{32} \rho_{31}-\rho_{12}\right]=0 \\
& c_{31} \sigma_{1} \sigma_{3}\left|\mathbf{h}_{1}\right|^{2}+c_{32} \sigma_{1} \sigma_{3} \mathbf{h}_{1}^{H} \mathbf{h}_{2}+c_{32} \sigma_{1} \sigma_{3} \mathbf{h}_{1}^{H} \mathbf{h}_{3}+\mu_{4} \rho_{13}+\mu_{3}\left[\rho_{12} \rho_{23}-\rho_{13}\right]=0 \\
& c_{31} \sigma_{2} \sigma_{3} \mathbf{h}_{2}^{H} \mathbf{h}_{1}+c_{32} \sigma_{2} \sigma_{3}\left|\mathbf{h}_{2}\right|^{2}+c_{32} \sigma_{2} \sigma_{3} \mathbf{h}_{2}^{H} \mathbf{h}_{3}+\mu_{5} \rho_{23}+\mu_{3}\left[\rho_{21} \rho_{13}-\rho_{23}\right]=0
\end{aligned}
$$

where $c_{i j}$ denotes the $(i, j)$ entry of $\operatorname{Adj}\left(\left(I_{3}+Q H^{H} H\right)\right)$ and are given in Appendix $\mathrm{C}$, and

$$
\begin{gathered}
\mu_{3}=\left\{\begin{array}{cc}
0 & \text { if } 1+2 \Re\left[\rho_{12} \rho_{23} \rho_{31}\right]-\left|\rho_{12}\right|^{2}-\left|\rho_{31}\right|^{2}-\left|\rho_{23}\right|^{2} \geq 0 ; \\
>0 & \text { if } 1+2 \Re\left[\rho_{12} \rho_{23} \rho_{31}\right]-\left|\rho_{12}\right|^{2}-\left|\rho_{31}\right|^{2}-\left|\rho_{23}\right|^{2}<0
\end{array}\right. \\
\mu_{4}= \begin{cases}0 & \text { if }\left|\rho_{13}\right|^{2} \leq 1 ; \\
>0 & \text { if }\left|\rho_{13}\right|^{2}=1\end{cases}
\end{gathered}
$$

and

$$
\mu_{5}= \begin{cases}0 & \text { if }\left|\rho_{23}\right|^{2} \leq 1 \\ >0 & \text { if }\left|\rho_{23}\right|^{2}=1\end{cases}
$$




\section{Random Channel Matrix}

It is well-known that the capacity of a flat fading channel with perfect channel state information at the transmitter (CSIT) and perfect channel state information at the receiver (CSIR) is the average of the maximum mutual information for each channel realization; hence the expression in (5.10) should apply for each fading state. On the other hand, with perfect CSIR only, the ergodic capacity, is given as [85]

$$
C=\max _{Q} E_{\mathbf{H}}\left[\log \operatorname{det}\left[I_{2}+Q \mathbf{H}^{H} \mathbf{H}\right]\right]
$$

The optimal $Q$ is dependent on the stationary distribution of the channel process. One way to approximate the capacity is to optimize for the Jensen's upper-bound on the mutual information obtained by using Jensen's inequality [93, 94] as

$$
E_{\mathbf{H}}\left[\log \operatorname{det}\left[I_{2}+Q \mathbf{H}^{H} \mathbf{H}\right]\right] \leq \log \operatorname{det}\left[I_{2}+Q E_{\mathbf{H}}\left[\mathbf{H}^{H} \mathbf{H}\right]\right]
$$

Then similar to the expression in (5.16), we may express the optimal input correlation coefficient for $L=2$ as

$$
\rho_{12}=\frac{E\left[\mathbf{h}_{\mathbf{1}}^{\mathbf{H}} \mathbf{h}_{\mathbf{2}}\right]}{\sqrt{\frac{P^{2}}{4}-\Delta_{2}^{2}}\left[E\left|\mathbf{h}_{\mathbf{1}}\right|^{2} \mathbf{E}\left|\mathbf{h}_{\mathbf{2}}\right|^{\mathbf{2}}-\mathbf{E}\left[\left|\mathbf{h}_{\mathbf{1}}^{\mathbf{H}} \mathbf{h}_{\mathbf{2}}\right|^{\mathbf{2}}\right]\right]+\mu_{1}}, \text { for } 0<\Delta_{2}<\frac{P}{2}
$$

Moreover, an analogous form of Proposition 5.1 will follow.

\subsection{Conclusions}

The design of DAS (D-MIMO) systems has motivated the problem of characterizing the capacity of MIMO channels with individual power constraints. In this chapter, the design of the optimal input covariance matrix in a Gaussian MIMO channel with both common and individual power constraint is considered. Carrying out the optimization 
analytically using the standard Lagrangian approach has led to an explicit expression, in terms of the difference between the allocated powers and the channel correlations, for the range of input SNR for which beamforming is optimal in TIMO channels with individual power constraints. The results are extended to random channels using Jensen's upper bound on mutual information. Moreover, the obtained expressions explain the observation that the capacity of correlated TIMO channels can exceed the capacity of uncorrelated TIMO channels at low SNR for TIMO channels with common power constraints and highlight the limitations on the beamforming for TIMO channels with individual power constraints. 


\section{Chapter 6}

\section{On the Capacity of Distributed Antenna Systems in Cellular Networks}

As discussed in the thesis introduction, the performance analysis of different communication schemes in DASs is dependent on the underlying channel model; hence, in this chapter the applications of the results obtained previously, for the generalized- $K$ model, to different performance metrics such as the ergodic capacity and the information outage probability are presented.

In this chapter, the performance of DASs for single-user schemes in single-cell and multi-cell environments is considered. The deployment of the DAPs is based on centralized architectures. In centralized architectures, all the antenna ports are individually connected via dedicated links to the BS. On the other hand, in seriallydistributed architectures, the DAPs are connected to each other serially and only the last "leading" antenna port is connected to the BS. The adoption of centralized architectures is motivated by (i) the amount of signal processing at the DAPs should be kept minimal, (ii) the fiber-optic links have large capacity and immunity that enable conveying the received signals to the BS. ${ }^{1}$

\footnotetext{
${ }^{1}$ The assumption of an ideal delay-less and infinite information capacity wired medium (backbone) may be reconsidered and proposals on more effective utilization of the bandwidth of the wired part of the network were introduced [95].
} 


\subsection{Related Literature}

The single-user ergodic channel capacity of DASs and the sum rate of a multi-user DAS in single-cell environments were investigated and compared with those of collocated antenna systems (CAS) using Monte Carlo simulation in [18] and [19], respectively, where improvements of the outage capacity have been observed which is due to the independent shadowing statistics (macro-diversity) at the distributed ports. In [96], the performance of optimum microscopic combining, which takes into account interference suppression, and macroscopic MRC in multi-cell environments is compared. It was shown, by simulations, that microscopic combining outperforms macroscopic MRC in high interference scenarios. In [97], the ergodic capacity of both selective transmission (where just one or two of the distributed antennas are used) and blanket transmission (where all antennas in the cell broadcast data) is analyzed using the lognormal-based composite fading model. The main conclusion is that selective transmission outperforms blanket transmission in multicell environments since it reduces the total interference experienced by each UT. A similar analysis was carried out in [98] where the performance of a transmission scheme that is based on the cooperation of a subset of cooperating DAPs is compared to the performance of single-antenna selective transmission. It was found that there are "cross-over" points among the different cooperative schemes and an adaptive cooperation scheme where the number of the cooperating DAPs is changed according to the received signal-tointerference ratio (SIR). The obtained results were again mainly based on simulations due the use of the lognormal-based fading model.

\subsection{Centralized DAS Architectures}

A centralized architecture is shown in Fig. 6.1 where a set of $N$ DAPs are deployed in an arrangement that is determined by the scatterers layout, the user density, etc. 


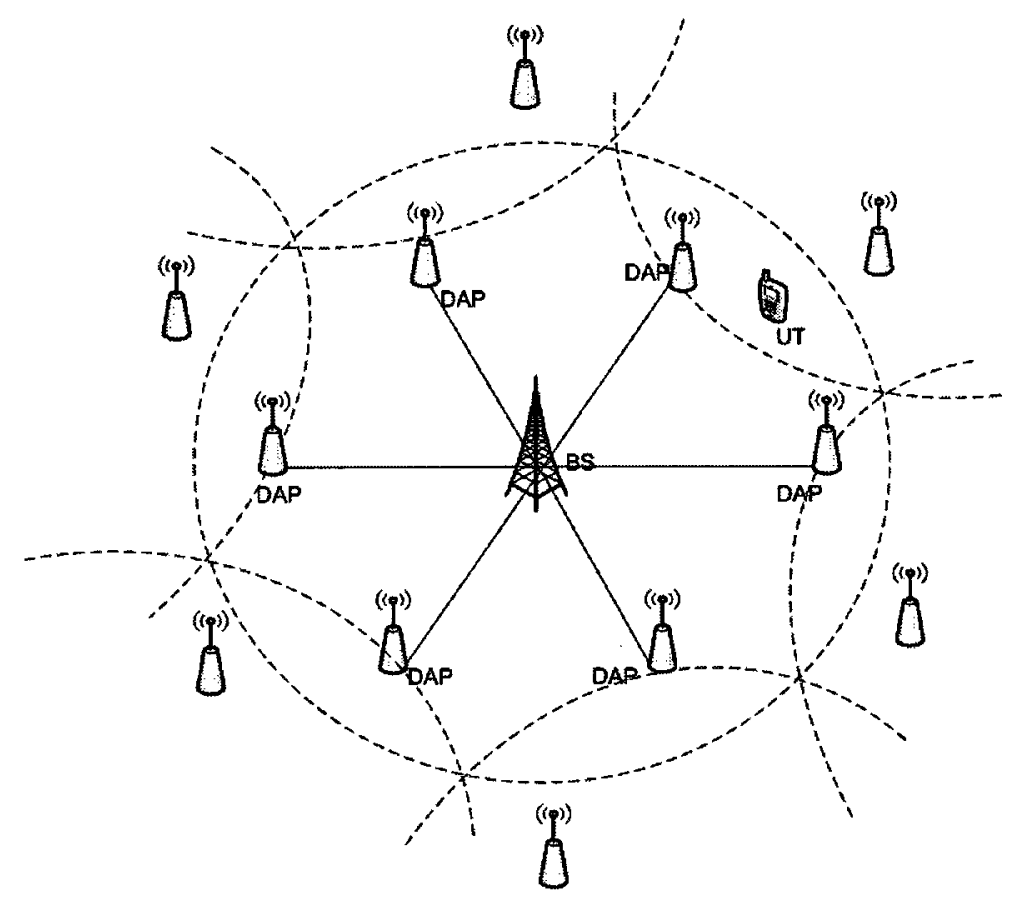

Figure 6.1: A multicell DAS with six DAPs per cell.

All the DAPs are assumed to convey their received signals to the central processor via the dedicated fiber-optic links. In general, the user terminal and each distributed port have $M$ and $L$ antenna elements, respectively. This system is also known as the distributed MIMO system [16] where the differences from the collocated MIMO system, as discussed in Chapter 1, are on the channel statistics, the power constraints, and the wired medium (backbone) constraints. Again the latter one is not considered here since the fiber optic links and network backbone are assumed to be noise-less and delay-less. 


\subsection{The Performance of DAS in a Single-cell Sce- nario}

\subsubsection{The Signal and Channel Model}

The received signal at the BS, through the set of the cooperating (participating) DAPs, can be expressed as

$$
\mathbf{y}=\left(\begin{array}{c}
\sqrt{\frac{w_{1}}{r_{1}}} h_{1} \\
\vdots \\
\sqrt{\frac{w_{N_{c p}}}{r_{N_{c p}}}} h_{N_{c p}}
\end{array}\right) x+\mathbf{n}
$$

where $h_{i}$ for $i=1, \ldots, N_{c p}$ denotes the small-scale (multipath) channel coefficient from the UT to the $i$ th DAP, $w_{i}$ is the shadowing component, $\beta$ is the path loss exponent (typically ranging from 2 to 4 ), $x$ is the transmitted signal and $\mathbf{n}$ is the $N_{c p} \times 1$ zeromean complex additive white Gaussian noise vector distributed as $\mathcal{N}\left(0, \sigma_{n}^{2} I_{N_{c p}}\right)$. The number of cooperating DAPs is $N_{c p} \subseteq N$. For analytical tractability, the path loss exponent is assumed to be the same for all cooperating antennas. Such an assumption is reasonable since the cooperating DAPs are expected to lie in the same environment (typical urban, suburban, etc.).

The normalized distance $r$ can be expressed as

$$
r_{i}=\left(\frac{d_{B S}}{d_{1}}\right)^{\beta}\left(\frac{d_{1}}{d_{i}}\right)^{\beta}, i=1, \ldots, N_{c p}
$$

where $d_{i}$ denotes the distance between the UT and the $i$ th DAP where the minimum distance (to the nearest DAP) is denoted as $d_{1}$, and $d_{B S}$ denotes the distance between the UT and the BS which is included to reveal the gain of reducing the access distance when the UT is far away from the BS but near one or more of the DAPs. The ratios 
$\left(\frac{d_{B S}}{d_{1}}\right)^{\beta}$ and $\left(\frac{d_{1}}{d_{i}}\right)^{\beta}$ will be used in the selection criterion of the cooperating DAPs as described later.

The envelope of the fading signal, $|h|$, is modeled by the versatile Nakagami distribution due to its applicability in different propagation environments where the fading statistics between the UT and nearby DAPs are expected to follow a Nakagami or Rician distribution rather than a Rayleigh one due to the higher probability of having a line-of-sight component or strong specular components (i.e., $m_{m}>1$ ). So, using the expression in (3.5), we may write

$$
p_{|h|}(x)=\frac{2}{\Gamma\left(m_{m}\right)}\left(\frac{m_{m}}{\Omega}\right)^{m_{m}} x^{2 m_{m}-1} \exp \left(-\frac{m_{m} x^{2}}{\Omega}\right)
$$

\subsubsection{The Ergodic Capacity and the Information Outage Ca- pacity of a Single-user DAS Over Independent Generalized- $K$ Channels}

For the single-user case with a single antenna and single-antenna ports, the equivalent

model is a distributed single-input multiple-output (D-SIMO) channel whose ergodic capacity, assuming an ergodic channel and a long coding period, can be expressed as

$$
C_{e r g}=E\left[\log _{2}\left(1+\operatorname{SNR}\left(\frac{d_{B S}}{d_{1}}\right)^{\beta} \sum_{i=1}^{N_{c p}}\left(\frac{d_{1}}{d_{i}}\right)^{\beta} z_{i} w_{i}\right)\right]
$$

where $z_{i}=\left|h_{i}\right|^{2}$ for $i=1, \ldots, N_{c p}$, and SNR is the input signal-to-noise ratio $P / \sigma_{n}^{2}$.

On the other hand, if coding is over only a small number of the channel coherence periods, then one needs to resort to the notion of the probability of outage for a target rate $R$, which can be expressed as

$$
P_{\text {out }}(R)=P\left(\log _{2}\left[1+\operatorname{SNR}\left(\frac{d_{B S}}{d_{1}}\right)^{\beta} \sum_{i=1}^{N_{c p}}\left(\frac{d_{1}}{d_{i}}\right)^{\beta} z_{i} w_{i}\right] \leq R\right)
$$


Clearly, both performance measures are dependent on the distribution of the following

$$
\zeta_{w}=\sum_{i=1}^{N}\left(\frac{d_{1}}{d_{i}}\right)^{\beta} z_{i} w_{i}
$$

As shown in Chapter 4, the PDF of the weighted sum of independent $N$ generalized$K$ RVs, in (4.15), can be well-approximated by another $N$ generalized- $K$ PDF using the moment matching method and that the approximation improves as the weights get equal. So, for the DASs set-up considered, since the participating (cooperating) set of DAPs contains only the ones having relatively strong weights (small access distances), the approximation accuracy, without adjustment, will be sufficient to approximate or lower bound the CDF of the weighted sum of $N$ generalized- $K$ RVs.

So, we may express the ergodic capacity, using [33, Eqn. 7.811.1], as

$$
C_{e r g}=\frac{1}{\operatorname{In}(2) \Gamma\left(m_{m, \tilde{\zeta_{w}}}\right) \Gamma\left(m_{s, \tilde{\zeta_{w}}}\right)} G_{2,4}^{4,1}\left[\left.\frac{m_{m, \tilde{\zeta_{w}}} m_{s, \tilde{\zeta w}}}{\Omega_{0, \tilde{\zeta_{w}}} \mathrm{SNR}_{e}} x\right|_{0,0, m_{m, \zeta \bar{w}}, m_{m, \tilde{\zeta}}} ^{0,1}\right]
$$

where $\mathrm{SNR}_{e}=\frac{P}{\sigma_{n}^{2}}\left(\frac{d_{B S}}{d_{1}}\right)^{\beta}$. The expression in (6.7) is simpler to obtain and more compact than the one in [49, Eqn. 7]. The information outage probability versus $R$ can be derived, using [51, Eqn. 26], as

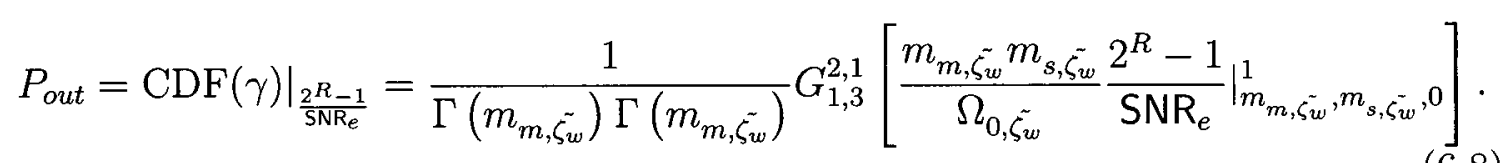

The previous results can be extended to the case where each of the distributed ports has multiple antennas ( $L$ of them); in that case, the output, after MRC, can be expressed as

$$
\gamma_{t}=\sum_{i=1}^{N_{c p}}\left(\frac{d_{1}}{d_{i}}\right)^{\beta} \sum_{l=1}^{L} \gamma_{l, i}
$$

However, since the signals received at each port experience the same shadowing statistics, it is straightforward to show that each $\gamma_{p}=\sum_{l=1}^{L} \gamma_{l}$ has a generalized- $K$ PDF with 
$m_{m, p}=L m_{m}, m_{s, p}=m_{s}$, and $\Omega_{0, p}=L \Omega_{0}$. Hence, the expression in (6.9) reduces to

$$
\gamma_{t}=\sum_{i=1}^{N_{c p}}\left(\frac{d_{1}}{d_{i}}\right)^{\beta} \gamma_{p, i}
$$

In Figs. 6.2 and 6.3, the plots of the information outage probability versus the target rate and the ergodic capacity of the DAS system, as compared to the CAS (where, in general, $N_{B S}$ antennas are collocated at the BS), demonstrate the attained gains due to reduced access distance (as seen from the CAS curve as compared to the DAS one for $N_{c p}=1$ ), better multipath fading conditions and macrodiversity (the improvement attained from $N_{c p}=1$ to $N_{c p}=4$ ), and that most of this gain is attained through three cooperating DAPs only. For both plots, we set $m_{m}=2$ for the DAS and $m_{m}=1$ for the CAS, $m_{s}=2\left(\sigma_{s}=5.5 \mathrm{~dB}\right)$, and $\beta=3$ for both systems. Note that for the collocated case, it can be shown that the sum, at the BS, has a generalized- $K$ PDF with $m_{m, \text { sum }}=N_{B S} m_{m}$ (assuming that the multipath components are independent), $m_{s, \text { sum }}=m_{s}$ (since fully correlated shadowing is experienced), and $\Omega_{0, \text { sum }}=N_{B S} \Omega_{0}$. To demonstrate the accuracy of the approximate ergodic capacity and information outage probability expressions, the obtained curves in Figs. 6.2-3 (for $N_{c p}=2$ and $\left.N_{c p}=4\right)$ are plotted with the simulation-based curves in Fig. 6.4-5.

Remark: For a multi-antenna UT, (i.e., $M>1$ ), lower and upper bounds on the mutual information in [99, Proposition 4] and [100, Appendix B] can be computed using the introduced approximation for the PDF of the weighted sum of generalized$K \mathrm{RVs}$ since both bounds are dependent on the squared Frobenius norm $\|G\|_{F}^{2}$ which can be expressed as

$$
\|G\|_{F}^{2}=\operatorname{tr}\left(G^{H} G\right)=\sum_{i=1}^{N_{c p}}\left(\frac{d_{1}}{d_{i}}\right)^{\beta} \sum_{j=1}^{M} \gamma_{i, j}
$$

where $G$ denotes the $N_{c p} \times M$ channel gain matrix from the UT to the $N_{c p}$ cooperating 


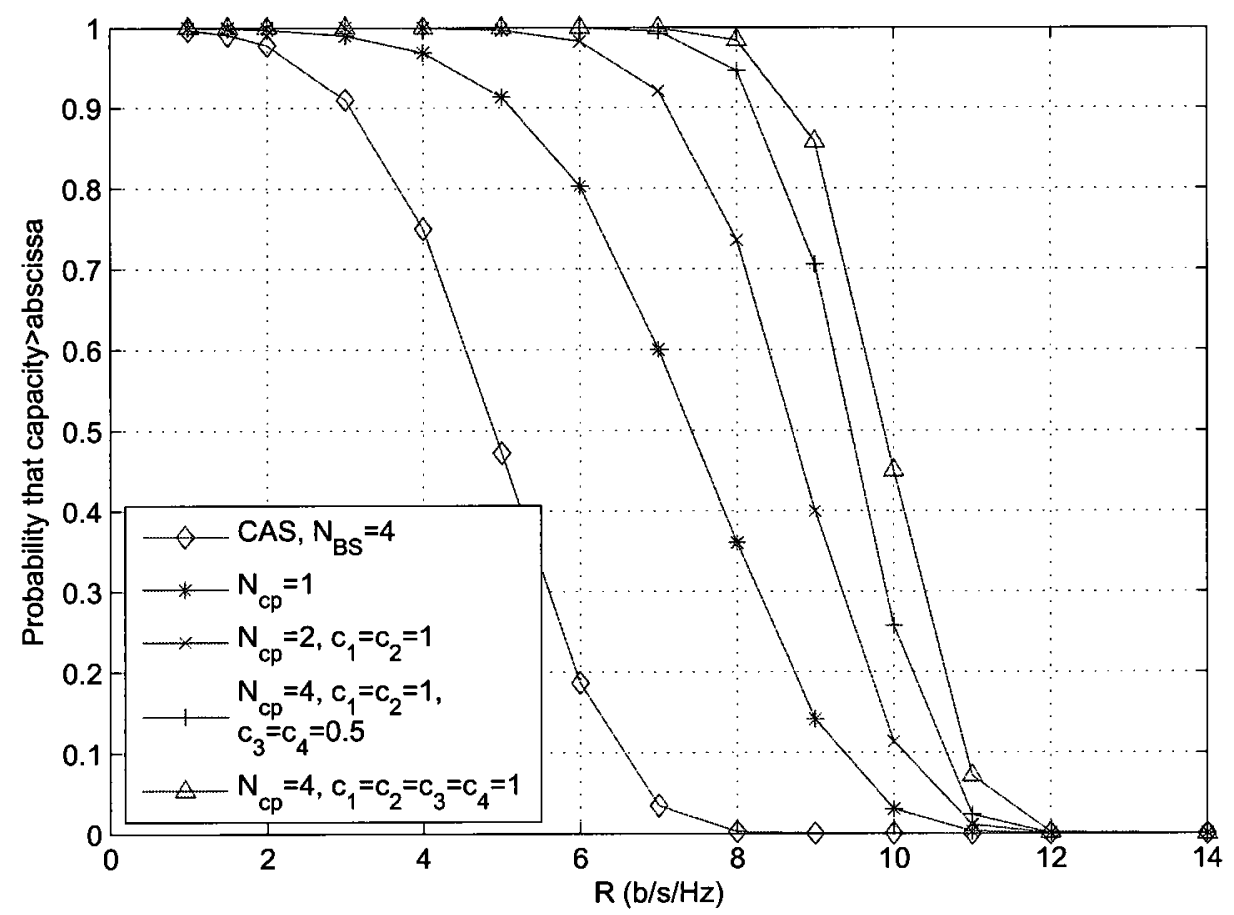

Figure 6.2: The plot of the outage probability versus the target rate for different numbers of cooperating DAPs at $P / \sigma^{2}=10 \mathrm{~dB}$ where the $c_{i}^{\prime} s$ are as defined in (4.15) such that $c_{i}=\left(\frac{d_{1}}{d_{i}}\right)^{\beta}$. 


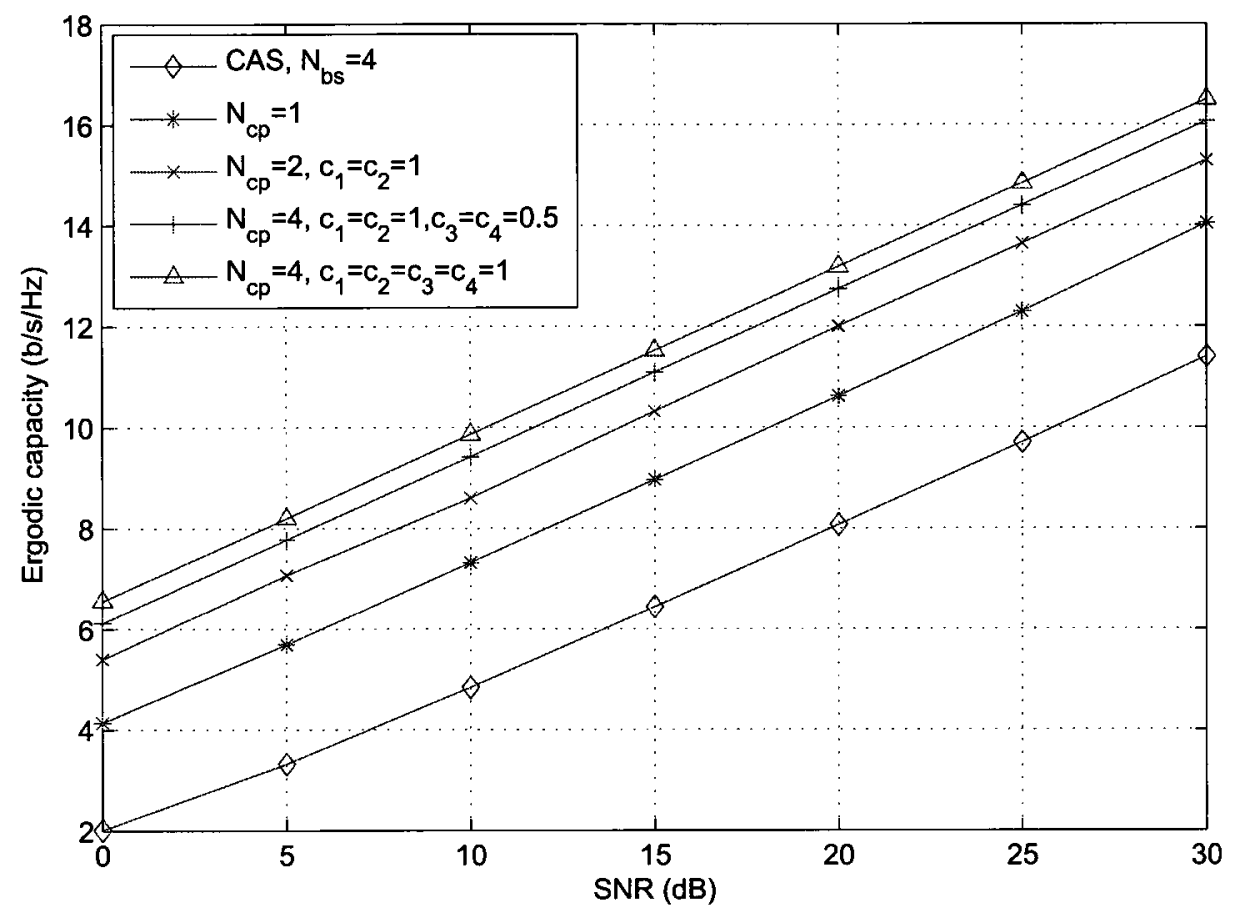

Figure 6.3: The plot of the ergodic capacity for different numbers of cooperating DAPs.

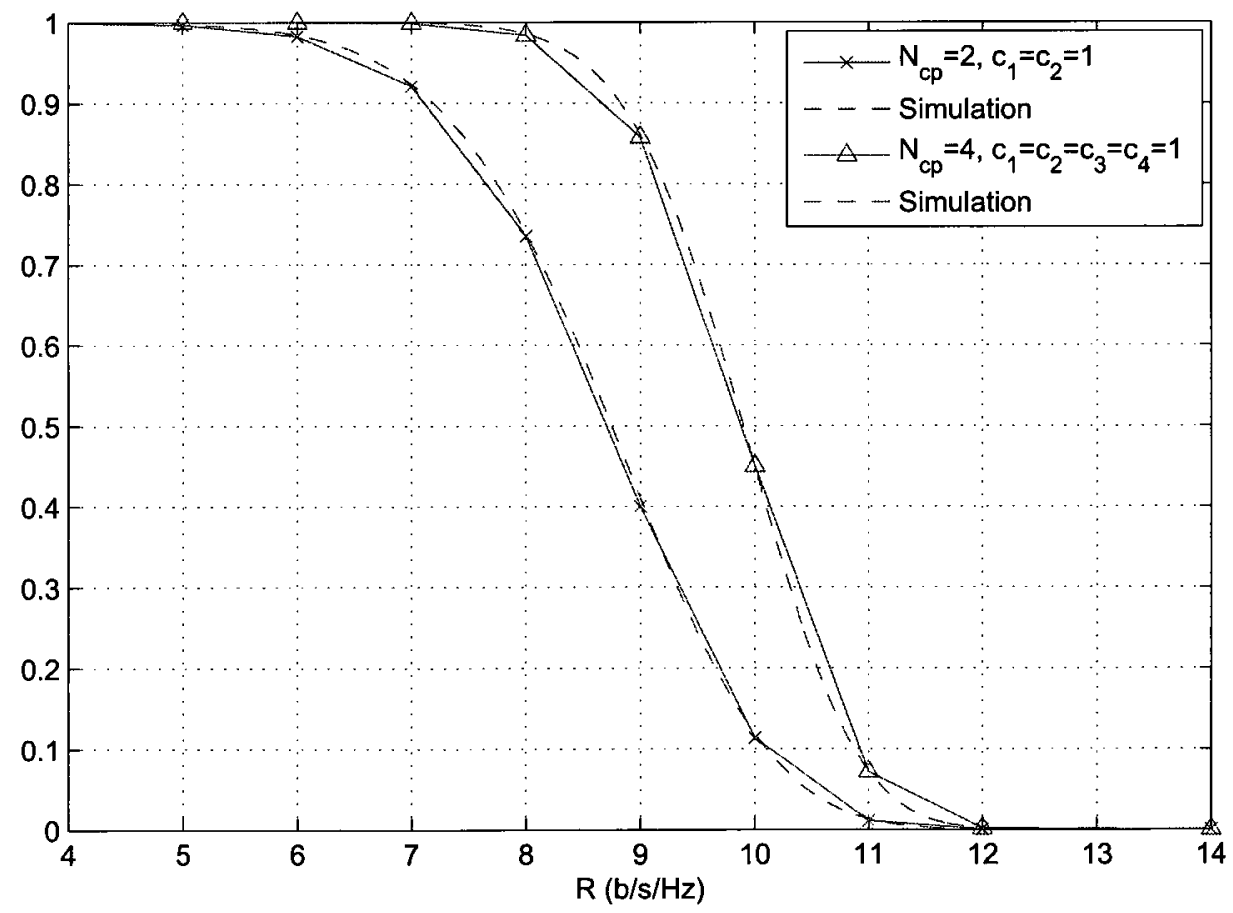

Figure 6.4: The plot of the approximate and actual information outage probability versus the target rate for different numbers of cooperating DAPs. 


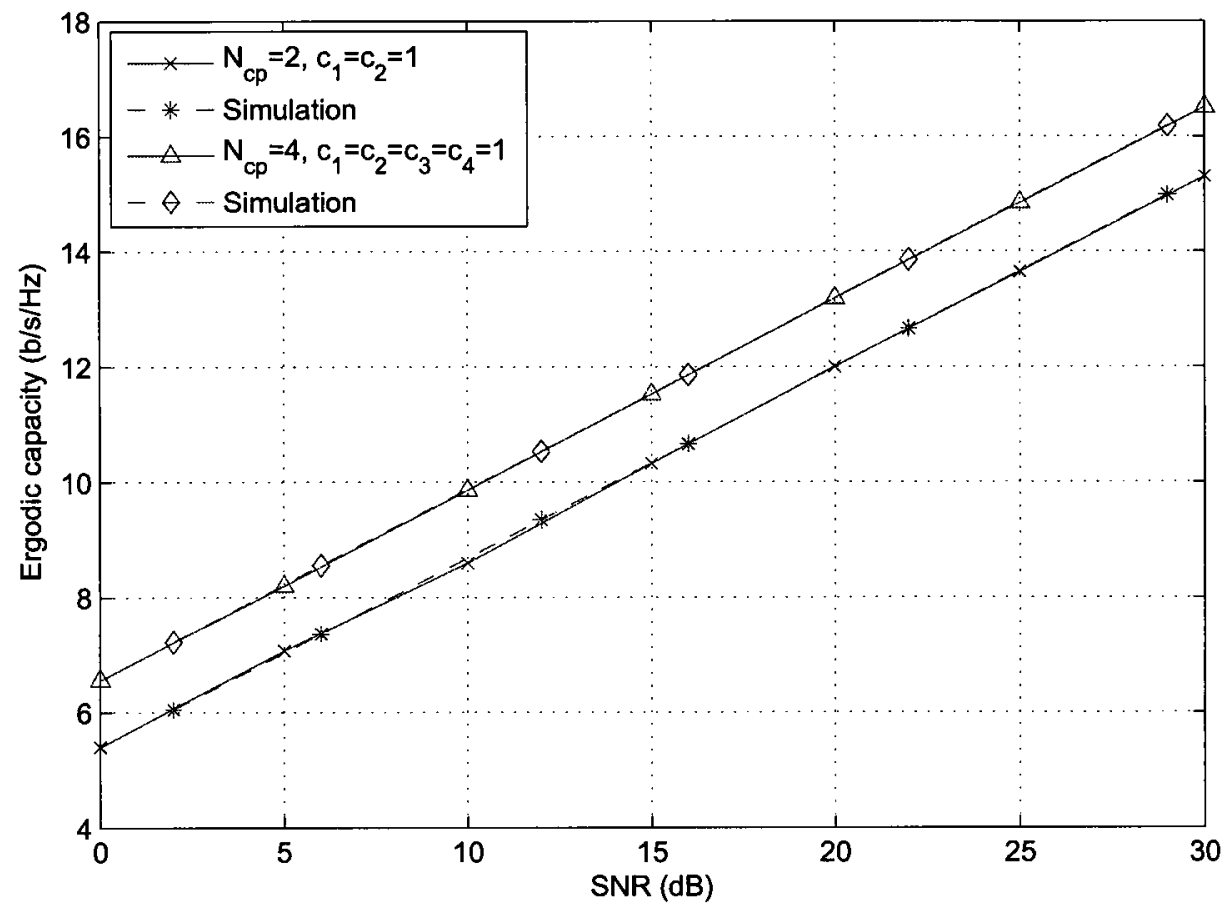

Figure 6.5: The plot of the approximate and actual ergodic capacity for different numbers of cooperating DAPs.

DAPs which include the effect of multipath fading, shadowing and the relative access distance ratios. Again the approximate PDF of the double summation in (6.11) can be obtained in a two-step approach as described before for the one in (6.9).

For the downlink scenario, the information capacity formulation is different from the classical collocated MISO channel due to the individual power constraint at each DAP. We may consider the case of $N_{c p}=2$ where the mutual information of the equivalent D-MISO channel can be expressed as

$$
\begin{aligned}
& I(x ; y)= \\
& \log _{2}\left(1+\frac{1}{\sigma_{n}^{2}}\left(\frac{d_{B S}}{d_{1}}\right)^{\beta}\left(\sigma_{1}^{2}\left|h_{1}\right|^{2}+\left(\frac{d_{1}}{d_{2}}\right)^{\beta} \sigma_{2}^{2}\left|h_{2}\right|^{2}+\left(\frac{d_{B S}}{d_{1}}\right)^{-\beta / 2}\left(\frac{d_{1}}{d_{2}}\right)^{\beta / 2} \sigma_{1} \sigma_{2} \kappa_{1}\right)\right),
\end{aligned}
$$

where $h_{i}$ denotes the $i$ th entry of the channel vector between the two transmitting 
DAPs and the UT, $\sigma_{1}$ and $\sigma_{2}$ denote the allocated powers at the two transmit DAPs, and $\kappa_{1}=\left[\rho_{21} h_{2} h_{1}^{*}+\rho_{12} h_{1} h_{2}^{*}\right]$. Now, if full CSI is available at the transmitter, then we may set $\rho_{12}=\frac{h_{2} h_{1}^{*}}{\left|h_{1}\right|\left|h_{2}\right|}$ and the instantaneous channel capacity can be expressed as

$$
\begin{aligned}
& C= \\
& \log _{2}\left(1+\frac{1}{\sigma_{n}^{2}}\left(\frac{d_{B S}}{d_{1}}\right)^{\beta}\left(\sigma_{1}^{2}\left|h_{1}\right|^{2}+\left(\frac{d_{1}}{d_{2}}\right)^{\beta} \sigma_{2}^{2}\left|h_{2}\right|^{2}+\left(\frac{d_{B S}}{d_{1}}\right)^{-\beta / 2}\left(\frac{d_{1}}{d_{2}}\right)^{\beta / 2} \sigma_{1} \sigma_{2} \kappa_{2}\right)\right),
\end{aligned}
$$

where $\kappa_{2}=2\left|h_{1}\right|\left|h_{2}\right|$.

A lower bound on the achievable ergodic capacity corresponds to the case where no CSI is available at the transmitter where the ergodic capacity is

$$
C_{\text {erg }}=E\left[\log _{2}\left(1+\frac{1}{\sigma_{n}^{2}}\left(\frac{d_{B S}}{d_{1}}\right)^{\beta}\left(\sigma_{1}^{2}\left|h_{1}\right|^{2}+\left(\frac{d_{1}}{d_{2}}\right)^{\beta} \sigma_{2}^{2}\left|h_{2}\right|^{2}\right)\right)\right]
$$

which again can be computed using the proposed approximation for the weighted sum of $N$ generalized- $K \mathrm{RVs}$.

\subsection{The Performance of DASs in Multi-cell Envi- ronments}

In a multi-cell DAS consisting of $B_{b}$ BSs with $N$ DAPS per cell, the downlink signal received at the single user in the 0th cell, for $N_{c p}$ cooperating DAPS, can be expressed as

$$
y=\left(\begin{array}{c}
\sqrt{\frac{w_{1,0}}{r_{1,0}}} h_{1,0} \\
\vdots \\
\sqrt{\frac{w_{N_{c p}, 0}}{r_{N, 0}}} h_{N_{c p}, 0}
\end{array}\right)^{T} x+\sum_{j=1}^{B_{I}}\left(\begin{array}{c}
\sqrt{\frac{w_{1, j}}{r_{1, j}}} h_{1, j} \\
\vdots \\
\sqrt{\frac{w_{N^{\prime}, j}}{r_{N^{\prime}, j}}} h_{N^{\prime}, j}
\end{array}\right)^{T} x_{j}+n
$$


where

$$
r_{i, j}=\left(\frac{d_{B S, j}}{d_{1, j}}\right)^{\beta_{j}}\left(\frac{d_{1, j}}{d_{i, j}}\right)^{\beta_{j}}, \text { for } i=1, \ldots, N^{\prime}
$$

where $d_{i, j}$ denotes distance between the UT and the $i$ th DAP in the $j$ th cell with a minimum of $d_{1, j}, d_{B S, j}$ denotes the distance between the UT and the $j$ th BS, which is included to reveal the effect of reducing the access distance, and $N^{\prime}$ denotes the number of active DAPs in interfering cells $\left(N^{\prime}=N_{c p}\right.$ for the 0 th cell).

The signal-to-interference ratio (SIR), where the role of thermal noise is neglected (for interference-limited scenarios) and equal power allocation among the DAPs in all the cells is assumed, for the single user at the 0th cell can be expressed, through extending the expression in [98, Eqn. 4], as

$$
\gamma_{m c, 0}=\frac{\left(\frac{d_{B S, 0}}{d_{1,0}}\right)^{\beta} \sum_{i=1}^{N_{c p}}\left(\frac{d_{1,0}}{d_{i, 0}}\right)^{\beta} \gamma_{i}}{r d_{\max } \sum_{j=1}^{B_{1}}\left(\frac{d_{B S, j}}{d_{1, j}}\right)^{\beta_{j}} \sum_{i=1}^{N^{\prime}}\left(\frac{d_{1, j}}{d_{i, j}}\right)^{\beta_{j}} \gamma_{i, j}}
$$

where $B_{I} \leq B_{b}-1$ denotes the cardinality of the subset of interfering BSs and $r d_{\max }=\max \left(\frac{d_{B S, j}}{d_{1, j}}\right)^{\beta_{j}}$, for $j=1, \ldots, B_{I}$. In (6.17), it is assumed that the interfering signals from the $j$ th cell experience the same path loss exponent $\beta_{j}$. The expression in (6.17) indicates that while the integration of DAPs tends to enhance the desired signal (in the 0 th cell), it also enhances the inter-cell interference, by the factor $\left(\frac{d_{B S, j}}{d_{1, j}}\right)^{\beta_{j}}$, where the nearest interfering DAP, from the $j$ th cell, is almost always nearer to the desired user than the BS in that interfering cell.

Since each of the instantaneous powers of both the desired user and the interferers, in (6.15), has a generalized-K PDF, then the PDF of $\gamma_{m c, 0}$ can be obtained by approximating the PDF of the interference in the denominator by a generalized- $K$ PDF using a two-step approximation. In the first step, the PDF of the weighted sum of the $N^{\prime}$ generalized- $K$ RVs for each cell individually is approximated by another generalized- $K$ PDF and then, in the second step, the PDF of the weighted sum of the 
resulting $B_{I}$ generalized- $K \mathrm{RVs}$ is further approximated by another generalized- $K$ PDF. Finally, to get the approximate distribution of $\gamma_{m c, 0}$, we will need to determine the distribution of the quotient of two independent generalized- $K$ RVs; so, if we denote the quotient of two independent generalized- $K \mathrm{RVs}, X_{1}$ and $X_{2}$, whose parameters are $m_{m, 1}, m_{s, 1}$, and $\Omega_{0,1} ; m_{m, 2}, m_{s, 2}$, and $\Omega_{0,2}$, respectively, as $\chi=\frac{X_{1}}{X_{2}}$, then, using [50, Theorem 6.4.3], the PDF of $\chi$ can be expressed as (see appendix D)

$$
p(\chi)=d_{1} G_{2,2}^{2,2}\left[\left(\frac{m_{m, 1} m_{s, 1} \Omega_{0,2}}{m_{m, 2} m_{s, 2} \Omega_{0,1}}\right) \chi \|_{m_{m, 1}-1, m_{s, 1}-1}^{-m_{m, 2}-m_{s, 2}}\right], \chi>0
$$

where $d_{1}=\frac{m_{m, 1} m_{s, 1} \Omega_{0,2}}{\Gamma\left(m_{m, 1}\right) \Gamma\left(m_{s, 1}\right) \Gamma\left(m_{m, 2}\right) \Gamma\left(m_{s, 2}\right) \Omega_{0,1}}$. The expression in $(6.18)$ is more compact than the expression obtained in [53, Eqn. 15].

The corresponding achievable rates can be expressed as

$$
R_{a, j}=E\left[\log _{2}\left(1+\gamma_{m c, j}\right)\right], \text { for } j=1, \ldots, B_{b}
$$

and can be derived using [33, Eqn. 7.811.1]. Furthermore, the network throughput is given as

$$
T=\sum_{j=1}^{B_{b}} R_{a, j}
$$

Similarly, the corresponding information outage probability for the UT in the $j$ th cell can be expressed as

$$
P_{\text {out }}(R)=P\left(\log _{2}\left(1+\gamma_{m c, j}\right) \leq R\right)
$$

and can be derived using [51, Eqn. 26]. 


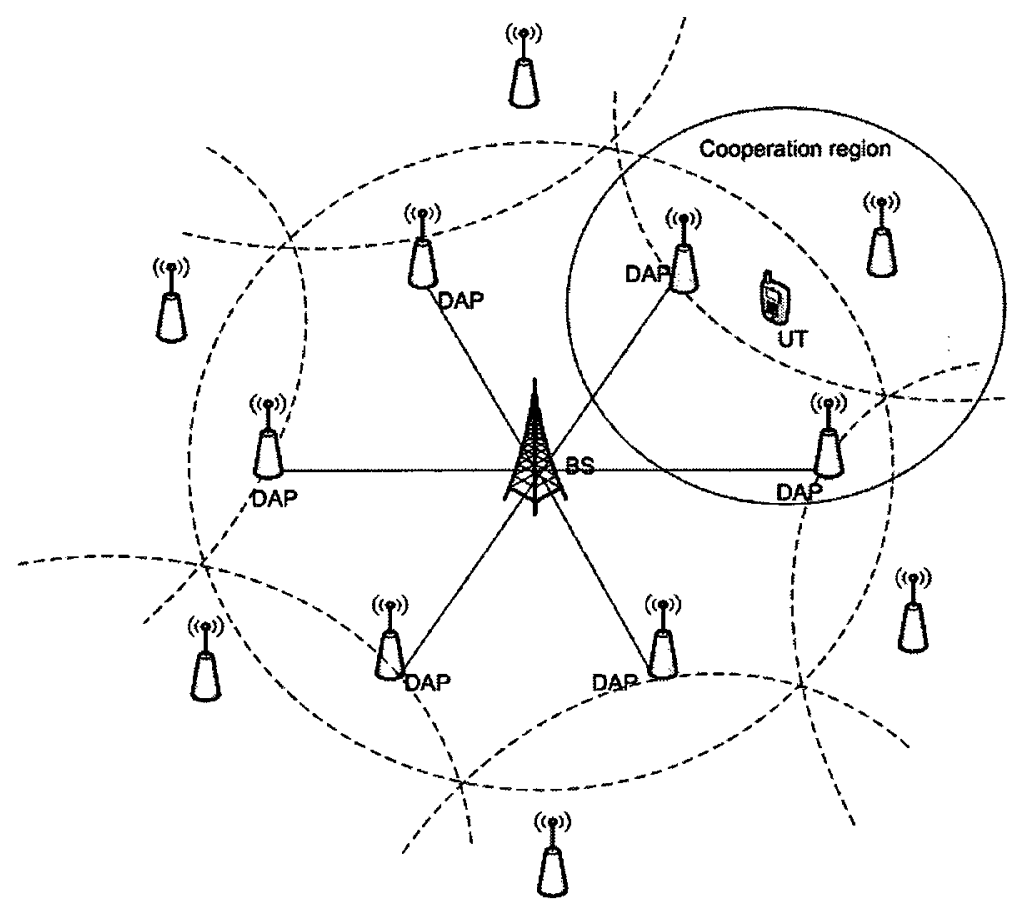

Figure 6.6: A cooperative multicell DAS with six DAPs per cell.

\subsubsection{Extension to the BSs Cooperative Schemes}

The developed expressions above for the ergodic capacity and the information outage probability can be extended to the scenario where a set of neighboring BSs may cooperate to transmit to their users jointly (instead of having each BS transmitting to its single user only). For example, a cell-edge UT can be served by its nearby DAPs being connected to $B_{c}$ cooperating BSs (including its own cell BS) as shown in Fig. 6.6. The SIR expression in (6.17) can be extended as 


$$
\begin{aligned}
& \gamma_{m c o o p, 0}= \\
& \frac{\left(\frac{d_{B S, 0}}{d_{1,0}}\right)^{\beta} \sum_{i=1}^{N_{c p}^{\prime}}\left(\frac{d_{1,0}}{d_{i, 0}}\right)^{\beta} \gamma_{i}+r d_{m a x, c} \sum_{j=1}^{B_{c}-1}\left(\frac{d_{B S, j}}{d_{1, j}^{(c p)}}\right)^{\beta_{j}} \sum_{i=1}^{N_{c p}^{\prime}}\left(\frac{d_{1, j}}{d_{i, j}}\right)^{\beta_{j}} \gamma_{i, j}}{r d_{\max , 1} \sum_{j=1}^{B_{I}}\left(\frac{d_{B S, j}}{d_{1, j}}\right)^{\beta_{j}} \sum_{i=1}^{N^{\prime}}\left(\frac{d_{1, j}}{d_{i, j}}\right)^{\beta_{j}} \gamma_{i, j}+r d_{\max , 2} \sum_{j=1}^{B_{c}-1}\left(\frac{d_{B S, j}}{d_{1, j}^{(\text {Scp }}}\right)^{\beta_{j}} \sum_{i=N_{c p}^{\prime}}^{N^{\prime}}\left(\frac{d_{1, j}}{d_{i, j}}\right)^{\beta_{j}} \gamma_{i, j}},
\end{aligned}
$$

where $N_{c p}^{\prime}$ denotes the number of the cooperating DAPs (i.e., sending to the desired $\mathrm{UT})$ in each of the $B_{c}$ cooperating cells, $d_{1, j}^{(c p)}$ and $d_{1, j}^{(n c p)}$ denote the distance from the UT to the nearest cooperating DAP and the nearest non-cooperating DAP in the $j$ th cooperating cell (since cooperating BSs transmit to their own users too), respectively. Subsequently, $r d_{\max , c}=\max \left(\frac{d_{B S, j}}{d_{1, j}^{(c)}}\right)^{\beta_{j}}$, for $j=1, \ldots, B_{c}-1$ and $r d_{\text {max }, 2}=\max \left(\frac{d_{B S, j}}{d_{1, j}^{n(p)}}\right)^{\beta_{j}}$, for $j=1, \ldots, B_{c}-1$.

The PDF of the expression in (6.22) can be approximated using the approach used before (for the one in (6.17)) and subsequent information capacity analysis can be carried out.

\subsection{Conclusions}

In past literature, capacity analysis of DASs in cellular networks was carried out using the intractable lognormal-based composite fading models where either numerical integration techniques or Monte Carlo simulations were used. In this chapter, the obtained results in previous chapters on the more tractable generalized- $K$ model, namely the approximate distributions for the sum and the weighted sum of independent generalized- $K$ RVs, are utilized to develop closed-form expressions for the ergodic capacity and the outage probability for DASs in both single-cell and multi-cell 
scenarios for both cooperative and non-cooperative schemes. The developed expressions can be used for computing the information outage probability or the ergodic capacity of centralized DAS architectures in cellular networks without resorting to extensive simulations. 


\section{Chapter 7}

\section{Conclusions and Future Research}

This thesis work was motivated by the new modeling problems that the emerging DASs (or D-MIMO systems) have spurred and the subsequent role of such models in the performance of these systems. In this thesis, we have developed some of the tools needed to model and further analyze the performance of DASs. First, the characterization of composite fading channels is considered where the generalized- $K$ composite fading model is introduced and an approximate Gamma model is proposed. Next, the statistics for the sum and the weighted sum of generalized- $K$ RVs are studied due to their essential role in the performance analysis of different trans-receiver schemes. Then, the design of the optimal input covariance matrix for MIMO channels with individual power constraint is considered. Finally, the developed results for the sum statistics are utilized to study the information capacity of DAS architectures in cellular networks.

\subsection{Summary and Conclusions}

After stating the motivations for the thesis work and giving some background material on some related topics to DASs, the generalized- $K$ composite fading model, which has been introduced as an alternative to the less tractable lognormal-base models, is presented and an approximation of the generalized- $K$ PDF by a Gamma PDF using 
the moment matching method is proposed. The obtained results indicate that the generalized- $K$ model can be approximated, in a certain region of interest, by the simple Gamma model. Such an approximation can be utilized in further performance analysis. For example, the ergodic capacity of the generalized- $K$ channel can be well-predicted by the approximating Gamma model.

In Chapter 4, two approximations for the distribution of the sum of independent generalized- $K$ RVs are proposed: The region-wise approximation using the Gamma distribution and the approximation of the sum distribution and the weighted sum distribution (in both the lower tail and upper tail regions) using another generalized$K$ distribution. The approximation accuracy is verified using the CDF plots and least square fit. In addition, the expression for the total correlation coefficient between two generalized- $K$ RVs in terms of the correlations among their multipath components and their shadowing components is derived, and then the expressions for the AF for the sum of correlated generalized- $K$ RVs are presented.

In Chapter 5, the standard Lagrangian formulation is utilized to derive the optimal correlation coefficient of the input covariance matrix for TIMO channels with individual power constraints. The range for beamforming optimality is expressed in terms of the input SNR, the channel correlation, and the disparity among the individual power constraints.

In Chapter 6, the applications of the results obtained in Chapter 4 on the statistics for the weighted sum of generalized- $K$ composite fading RVs to the information capacity of DASs in both single-cell and multi-cell scenarios are presented. The obtained expressions quantify the gains due reduced access distance, macrodiversity, and less severe multipath fading conditions in DASs. 


\subsection{Future Research Directions}

The work presented in the thesis can be extended in the following directions:

- For Chapter 3, further applications of the introduced approximation of the generalized- $K$ composite fading model by the Gamma model to the performance analysis of selection diversity schemes can be carried out since the CDF of the approximating Gamma distribution is much simpler to handle as compared to CDF of the generalized- $K$ distribution. Moreover, the introduced approximation can be extended to approximate the generalized- $K$ distribution by a mixture of Gamma PDFs using the theory on the moment-based approximation for the distribution of a univariate RV by a mixture of Gamma PDFs [101]. In this regard, an approximation of the Gamma-lognormal instantaneous composite fading power PDF by a mixture of Gamma PDFs was recently introduced in [102] and the fit accuracy of the proposed mixture distribution was compared with generalized- $K$ distribution.

- For Chapter 4, first, the exact distribution for the sum of independent generalized$K \mathrm{RVs}$, particularly for the case of the sum of two generalized- $K \mathrm{RVs}$, may be tackled through generalized Meijer function since the characteristic function of the sum, being the product of two Meijer functions leads to a generalized Meijer function with two arguments. However, up to the knowledge of the author, the inverse Laplace transform of the generalized Meijer function is not available. So, one may look for the possible representations, for some specific values, of the generalized Meijer function in terms of more familiar functions or for the use expansions that may lead to Laplace invertible expressions. Second, mathematical inequalities such as the arithmetic-geometric mean inequality and its weighted form; and Chebyshev inequality can be utilized to bound the CDFs of both the sum and the weighted sum distributions. 
- For Chapter 5, the Lagrangian approach gets tedious for a larger number of transmit antennas which suggests looking for an approach that would transform the optimization problem into a more tractable one.

- For Chapter 6, the developed approximate closed-form expressions can be used for further performance analysis particularly for the multicell scenario. This may include the performance analysis for the uplink where the random location and number of interfering users necessitates the use of a spatially stochastic model such as the Poisson model. Moreover, it is interesting for future cellular networks to further study the performance of DASs in cooperative cellular networks where a subset of the BSs do exchange information that would allow joint detection and hence reduce the effect of intercell interference. This would soften the constraints on the transmitted power from DAPs and allow higher network data rates. 
Appendices 


\section{Appendix A}

\section{The $H$-function Distribution Family}

Definition: The $H$-function can be defined as

$$
\mathbf{H}_{p, q}^{m, n}\left[\left.z\right|_{\left(b_{1}, \beta_{1}\right), \ldots,\left(b_{q}, \beta_{q}\right)} ^{\left(a_{1}, \alpha_{1}\right), \ldots,\left(a_{p}, \alpha_{p}\right)}\right]=\frac{1}{2 \pi i} \int_{C} \frac{\prod_{j=1}^{m} \Gamma\left(b_{j}-\beta_{j} s\right) \prod_{j=1}^{n} \Gamma\left(1-a_{j}+\alpha_{j} s\right)}{\prod_{j=m+1}^{q} \Gamma\left(1-b_{j}+\beta_{j} s\right) \prod_{j=n+1}^{p} \Gamma\left(a_{j}-\alpha_{j} s\right)} z^{s} d s
$$

where $0 \leq m \leq q, 0 \leq n \leq p, \alpha_{j}>0, \beta_{j}>0$. and $a_{j}(j=1,2, \ldots . p)$ and $b_{j}$ $(j=1,2, \ldots . q)$ are complex numbers such that no pole of $\Gamma\left(b_{j}-\beta_{j} s\right)$ for $j=1,2, \ldots m$ coincides with any pole of $\Gamma\left(1-a_{j}+\alpha_{j} s\right)$ for $j=1,2, \ldots, n$. The contour $C$ is a straight line parallel to the imaginary axis in the complex plane and the poles of $\Gamma\left(b_{j}-\beta_{j} s\right)$ lie on the right of $C$ while those of $\Gamma\left(1-a_{j}+\alpha_{j} s\right)$ lie on the left of $C$.

Many of the so-called special functions are special cases of the $H$-function, including Gauss and confluent hypergeometric functions, MacRobert E-function, Meijer function, and Bessel functions [50, 103].

The Meijer function can be expressed as

$$
\mathbf{G}_{p, q}^{m, n}\left[\left.x\right|_{b_{1}, \ldots, b_{q}} ^{a_{1}, \ldots, a_{p}}\right]=\mathbf{H}_{p, q}^{m, n}\left[\left.z\right|_{\left(b_{1}, 1\right), \ldots,\left(b_{q}, 1\right)} ^{\left(a_{1}, 1\right), \ldots,\left(a_{p}, 1\right)}\right]
$$


Definition: The $H$-function distribution can be expressed as

$$
p(x)=k \mathbf{H}_{p, q}^{m, n}\left[\left.c x\right|_{\left(b_{1}, \beta_{1}\right), \ldots,\left(b_{q}, \beta_{q}\right)} ^{\left(a_{1}, \alpha_{1}\right), \ldots,\left(a_{p}, \alpha_{p}\right)}\right], x>0
$$

where $k$ and $c$ are the parameters of the distribution such that $\int_{0}^{\infty} p(x) d x=1$.

The characteristic function of the $H$-function distribution can be derived as

$$
\phi(t)=\frac{k}{c} \mathbf{H}_{q, p+1}^{n+1, m}\left[-\left.\frac{i}{c} x\right|_{\left(1-b_{1}-\beta_{1}, \beta_{1}\right), \ldots,\left(1-b_{q}-\beta_{q}, \beta_{q}\right)} ^{(0,1),\left(1-a_{1}-\alpha_{p}, \alpha_{p}\right)}\right], x>0
$$

Many non-negative distributions are special cases of the $H$-function including the Gamma distribution, the Weibull distribution, the Beta distribution, the Rayleigh distribution, and the general hypergeometric distribution. 


\section{Appendix B}

\section{Derivations for Chapter 4}

\section{B.1 Derivation of the Maximum Possible Negative Correlation Coefficient}

Although a sketchy proof of the statement on the maximum possible negative correlation coefficient was given in [104], a more systematic presentation of the proof is given here. First, consider the covariance matrix of a vector of $N$ RVs which can be expressed as

$$
Q=\left[\begin{array}{cccc}
\sigma_{1}^{2} & \rho_{1,2} \sigma_{1} \sigma_{2} & \ldots & \rho_{1, N} \sigma_{1} \sigma_{N} \\
\rho_{2,1} \sigma_{1} \sigma_{2} & \sigma_{2}^{2} & \ldots & \rho_{2, N} \sigma_{1} \sigma_{N} \\
\vdots & \vdots & \vdots & \vdots \\
\rho_{N, 1} \sigma_{1} \sigma_{N} & \ldots & \ldots & \sigma_{N}^{2}
\end{array}\right]
$$

On the other hand, the determinant of the following matrix, using the result in [76, Problem 6.2.14(b)]), can be expressed as

$$
\left|\begin{array}{ccccc}
\alpha & \beta & \beta & \ldots & \beta \\
\beta & \alpha & \beta & \ldots & \beta \\
\beta & \beta & \alpha & \ldots & \beta \\
\vdots & \vdots & \vdots & \ddots & \vdots
\end{array}\right|_{n \times n}= \begin{cases}(\alpha-\beta)^{n}\left(1+\frac{n \beta}{\alpha-\beta}\right) & \text { if } \alpha \neq \beta \\
0 & \text { if } \alpha=\beta .\end{cases}
$$


Now considering the equally correlated case (i.e., $\rho_{i, j}=\rho$ for $i, j=1, \ldots, N$ and $i \neq$ $j$ ), we may set, without loss of generality, the variances to unity (i.e., $\alpha=1$ ) and $\beta=\rho$ and equate the determinant to zero to result in

$$
(1-\rho)^{n}\left(1+\frac{n \rho}{1-\rho}\right)=0 .
$$

Since $(1-\rho)^{n}$ is always positive, we may write $\left(1+\frac{n \rho}{1-\rho}\right)=0$ from which we get $\rho=\frac{-1}{N-1}$ and the statement follows. The geometric reasoning of this observation was stated by the mathematician Bruno de Finitti in one of his papers that he had published in 1937 [105] as "we can always represent $n$ random numbers by $n$ vectors having a modulus equal to their standard deviations and forming - two by two - an angle whose cosine (in magnitude and sign) is measured by the correlation coefficient. Now, $n$ vectors can surely be orthogonal among each other (no-correlation case), or can form acute angles (positive correlation), but it is impossible that they form angles which are all obtuse (over a certain angle limit). For example, three vectors can form, among one another, angles of at most 120. In general $n$ vectors can form angles whose cosine is at most equal to - 1/(n-1). And, therefore, this is the maximum negative correlation coefficient that we can simultaneously observe among n random numbers."

\section{B.2 Derivation of (4.20)}

We may write the correlation coefficient between the $i$ th and the $j$ th generalized- $K$ RVs as

$$
\rho_{i, j}=\frac{E\left[z_{i} w_{i} z_{j} w_{j}\right]-E\left[z_{i} w_{i}\right] E\left[z_{j} w_{j}\right]}{\sigma_{z_{i} w_{i}} \sigma_{z_{j} w_{j}}}
$$


Since the multipath components are independent from the shadowing components, the term $E\left[z_{i} w_{i} z_{j} w_{j}\right]$ can be expressed as

$$
E\left[z_{i} w_{i} z_{j} w_{j}\right]=E\left[z_{i} z_{j}\right] E\left[w_{i} w_{j}\right]
$$

Substituting (B.4) in (B.3), and using $E\left[z_{i} z_{j}\right]=\rho_{z_{i}, z_{j}} \sigma_{z_{i}} \sigma_{z_{j}}+E\left[z_{i}\right] E\left[z_{j}\right]$ and $E\left[w_{i} w_{j}\right]=$ $\rho_{w_{i}, w_{j}} \sigma_{w_{i}} \sigma_{w_{j}}+E\left[w_{i}\right] E\left[w_{j}\right]$, result in

$$
\rho_{i, j}=\frac{\left[\rho_{z_{i}, z_{j}} \sigma_{z_{i}} \sigma_{z_{j}}+E\left[z_{i}\right] E\left[z_{j}\right]\right]\left[\rho_{w_{i}, w_{j}} \sigma_{w_{i}} \sigma_{w_{j}}+E\left[w_{i}\right] E\left[w_{j}\right]\right]-E\left[z_{i} w_{i}\right] E\left[z_{j} w_{j}\right]}{\sigma_{z_{i} w_{i}} \sigma_{z_{j} w_{j}}}
$$

Now, using the interpretation of the generalized- $K$ composite fading model as a multiplication of two Gamma RVs where the mean of the one corresponding to multipath fading is unity and the mean of the other corresponding to the shadowing is $\Omega_{0}$, we may write $E\left[z_{i}\right]=1, \sigma_{z_{i}}=\sqrt{\frac{1}{m_{m, i}}}, E\left[w_{i}\right]=\Omega_{0, i}, \sigma_{w_{i}}=\sqrt{\frac{\Omega_{0, i}^{2}}{m_{s, i}}}, E\left[z_{i} w_{i}\right]=\Omega_{0, i}$, and $\sigma_{z_{i} w_{i}}=\sqrt{\frac{\left(m_{m, i}+m_{s, i}+1\right) \Omega_{0, i}^{2}}{m_{m, i} m_{s, i}}}$. With the substitutions, (B.5) leads to the expression in $(4.20)$.

\section{B.3 Proof of Proposition 4.2}

The AF for the sum of $N$ correlated generalized- $K$ RVs can be expressed as

$$
\mathrm{AF}_{\xi}=\frac{\sum_{i=1}^{N} \mathrm{AF}_{i} \Omega_{0, i}^{2}+\sum_{i=1}^{N} \sum_{j=1, i \neq j}^{N} \rho_{i, j} \sqrt{\mathrm{AF}_{i}} \sqrt{\mathrm{AF}_{j}} \Omega_{0, i} \Omega_{0, j}}{\left(\sum_{i=1}^{N} \Omega_{0, i}\right)^{2}}
$$

Adopting the general exponential correlation model, we may write

$$
\rho_{i, j}=\rho_{0} \exp |i-j|
$$


where $\rho_{0}$ denotes the correlation coefficient between the adjacent antenna elements. Substituting in (B.6)

$$
\mathrm{AF}_{\xi}=\frac{\sum_{i=1}^{N} \mathrm{AF}_{i} \Omega_{0, i}^{2}+\sum_{i=1}^{N} \sum_{j=1, i \neq j}^{N} \rho_{0} \exp |i-j| \sqrt{\mathrm{AF}_{i}} \sqrt{\mathrm{AF}_{j}} \Omega_{0, i} \Omega_{0, j}}{\left(\sum_{i=1}^{N} \Omega_{0, i}\right)^{2}}
$$

Assuming i.i.d. composite fading conditions,

$$
\mathrm{AF}_{\xi}=\frac{\mathrm{AF}_{0}}{N}+\frac{\mathrm{AF}_{0} \sum_{i=1}^{N} \sum_{j=1, i \neq j}^{N} \rho_{0} \exp |i-j|}{N^{2}}
$$

For the constant correlation model $\left(\rho_{i, j}=\rho_{0}\right)$, the AF expression reduces further to

$$
\mathrm{AF}_{\xi}=\frac{\left(1+(N-1) \rho_{0}\right) \mathrm{AF}_{0}}{N}=\frac{\left(1-\rho_{0}\right)}{N} \mathrm{AF}_{0}+\rho_{0} \mathrm{AF}_{0}
$$

Now, using Lemma 1, we may substitute (4.20) in (B.10) to get (4.22). 


\section{Appendix $\mathrm{C}$}

\section{Derivations for Chapter 5}

The determinant of $\left[I_{2}+Q H^{H} H\right]$ can be written as

$$
\operatorname{det}\left[I_{2}+H^{H} H Q\right]=\operatorname{det}\left[\begin{array}{cc}
1+\sigma_{1}^{2}\left|\mathbf{h}_{1}\right|^{2}+\rho_{12} \mathbf{h}_{2}^{H} \mathbf{h}_{1} & \sigma_{1}^{2} \mathbf{h}_{1}^{H} \mathbf{h}_{2}+\rho_{12} \sigma_{1} \sigma_{2}\left|\mathbf{h}_{2}\right|^{2} \\
\rho_{21} \sigma_{1} \sigma_{2}\left|\mathbf{h}_{1}\right|^{2}+\sigma_{2}^{2} \mathbf{h}_{2}^{H} \mathbf{h}_{1} & 1+\rho_{21} \sigma_{1} \sigma_{2} \mathbf{h}_{1}^{H} \mathbf{h}_{2}+\sigma_{2}^{2}\left|\mathbf{h}_{2}\right|^{2}
\end{array}\right]
$$

The expression in (C.1) can be further expanded as

$$
\begin{aligned}
& \operatorname{det}\left[I_{2}+H^{H} H Q\right]=1+\sigma_{1}^{2}\left|\mathbf{h}_{1}\right|^{2} \\
& +\sigma_{2}^{2}\left|\mathbf{h}_{2}\right|^{2}+\sigma_{1} \sigma_{2}\left[\rho_{12} \mathbf{h}_{2}^{H} \mathbf{h}_{1}+\rho_{21} \mathbf{h}_{1}^{H} \mathbf{h}_{2}\right]+\sigma_{1}^{2} \sigma_{2}^{2}\left[\left|\mathbf{h}_{1}\right|^{2}\left|\mathbf{h}_{2}\right|^{2}-\left|\mathbf{h}_{1}^{H} \mathbf{h}_{2}\right|^{2}\right]\left[1-\rho_{12} \rho_{21}\right] .
\end{aligned}
$$

Since the logarithm function can be maximized by maximizing its argument [p. 278] [60], then we may derive the optimal $Q$ by considering the following reduced problem:

$$
\max _{Q} \operatorname{det}\left[I_{2}+Q H^{H} H\right] \text {, s.t. } \sigma_{1}^{2}+\sigma_{2}^{2}=P, \sigma_{1}^{2} \geq 0, \sigma_{2}^{2} \geq 0, \text { and }\left|\rho_{12}\right|^{2} \leq 1 \text {. }
$$


Using the Karush-Kuhn-Tucker conditions for the optimization problem, we get

$$
\begin{aligned}
& \sigma_{1}^{2}\left[\left|\mathbf{h}_{1}\right|^{2}+\sigma_{2}^{2}\left[\left|\mathbf{h}_{1}\right|^{2}\left|\mathbf{h}_{2}\right|^{2}-\left|\mathbf{h}_{1}^{H} \mathbf{h}_{2}\right|^{2}\right]\left(1-\rho_{12} \rho_{21}\right)-\nu\right]=-\sigma_{1} \sigma_{2} \Re\left[\rho_{12} \mathbf{h}_{2}^{H} \mathbf{h}_{1}\right],(C . \\
& \sigma_{2}^{2}\left[\left|\mathbf{h}_{2}\right|^{2}+\sigma_{1}^{2}\left[\left|\mathbf{h}_{1}\right|^{2}\left|\mathbf{h}_{2}\right|^{2}-\left|\mathbf{h}_{1}^{H} \mathbf{h}_{2}\right|^{2}\right]\left(1-\rho_{12} \rho_{21}\right)-\nu\right]=-\sigma_{1} \sigma_{2} \Re\left[\rho_{12} \mathbf{h}_{2}^{H} \mathbf{h}_{1}\right],(\mathrm{C}
\end{aligned}
$$

and

$$
\begin{gathered}
\rho_{12}=\frac{\mathbf{h}_{1}^{H} \mathbf{h}_{2}}{\sigma_{1} \sigma_{2}\left[\left|\mathbf{h}_{1}\right|^{2}\left|\mathbf{h}_{2}\right|^{2}-\left|\mathbf{h}_{1}^{H} \mathbf{h}_{2}\right|^{2}\right]+\mu_{1}}, \\
\rho_{21}=\rho_{12}^{*},
\end{gathered}
$$

where

$$
\mu_{1}= \begin{cases}0 & \text { if }\left|\rho_{12}\right|^{2}<1 \\ >0 & \text { if }\left|\rho_{12}\right|^{2}=1\end{cases}
$$

and $\nu$ is the Lagrange multiplier associated with the equality constraint. Note that non-negativity constraint for $\sigma_{1}$ and $\sigma_{2}$ is implicitly included in the expressions in (C.4a) and (C.4b). First, we observe that the optimal $\sigma_{1}$ and $\sigma_{2}$ have to be non-zero as far as $\mathbf{h}_{1}^{H} \mathbf{h}_{2} \neq 0$ which can be seen from the expressions in (C.2) and (C.5a). So, if we set $\sigma_{1}^{2}=\frac{P}{2}+\Delta$ and $\sigma_{2}^{2}=\frac{P}{2}-\Delta$, where $\Delta<\frac{P}{2}$, then the expression in (5.16) will follow from (C.5a). Furthermore, since $\mu_{1}>0$ for $\left|\rho_{12}\right|=1$ (i.e., when beamforming takes place), the expression in (5.18) will follow.

Second, for MIMO channels with a common power constraint, the expressions in (C.4a) and (C.4b) are involved to solve for $\sigma_{1}$ and $\sigma_{2}$, and determine $\Delta$; instead, we propose the following solution: we may first solve for $\sigma_{1}$ and $\sigma_{2}$ (by setting $\left|\rho_{12}\right|=0$ ) and then compute the corresponding value $\rho_{12}$ using (C.5a); however if the magnitude of $\rho_{12}$ becomes unity or one of the allocated powers comes out to be zero (which will violate the observation stated above), then we go back to (C.4a) and (C.4b) to solve for $\sigma_{1}$ and $\sigma_{2}$ with $\left|\rho_{12}\right|=1$. The intuition behind this solution is that as far as beamforming is not the optimal transmission strategy, we may consider 
two uncorrelated Gaussian inputs, determine the optimal allocated powers, and then introduce the optimal correlation between them. So, setting $\left|\rho_{12}\right|=0$ in (C.4a) and (C.4b) results in

$$
\begin{array}{r}
\left|\mathbf{h}_{1}\right|^{2}+\sigma_{2}^{2}\left[\left|\mathbf{h}_{1}\right|^{2}\left|\mathbf{h}_{2}\right|^{2}-\left|\mathbf{h}_{1}^{H} \mathbf{h}_{2}\right|^{2}\right]-\nu=0, \\
\left|\mathbf{h}_{2}\right|^{2}+\sigma_{1}^{2}\left[\left|\mathbf{h}_{1}\right|^{2}\left|\mathbf{h}_{2}\right|^{2}-\left|\mathbf{h}_{1}^{H} \mathbf{h}_{2}\right|^{2}\right]-\nu=0,
\end{array}
$$

from which we may straightforwardly get $\sigma_{1}^{2}=\frac{1}{2}\left[\frac{\left|\mathbf{h}_{1}\right|^{2}-\left|\mathbf{h}_{2}\right|^{2}}{\left[\left|\mathbf{h}_{1}\right|^{2}\left|\mathbf{h}_{2}\right|^{2}-\left|\mathbf{h}_{1}^{H} \mathbf{h}_{2}\right|^{2}\right]}+P\right]$ and $\sigma_{2}^{2}=$ $\frac{1}{2}\left[\frac{\left|\mathbf{h}_{2}\right|^{2}-\left|\mathbf{h}_{1}\right|^{2}}{\left[\left|\mathbf{h}_{1}\right|^{2}\left|\mathbf{h}_{2}\right|^{2}-\left|\mathbf{h}_{1}^{H} \mathbf{h}_{2}\right|^{2}\right]}+P\right]$. However, since $0<\sigma_{1}^{2}<P$ and $0<\sigma_{2}^{2}<P$, then based on these expressions, we may define

$$
\Delta_{1}=\frac{1}{2} \frac{\left|\mathbf{h}_{1}\right|^{2}-\left|\mathbf{h}_{2}\right|^{2}}{\left[\left|\mathbf{h}_{1}\right|^{2}\left|\mathbf{h}_{2}\right|^{2}-\left|\mathbf{h}_{1}^{H} \mathbf{h}_{2}\right|^{2}\right]}
$$

Now if $\Delta_{1}=\frac{P}{2}$, then a violation will take place since either $\sigma_{1}$ or $\sigma_{2}$ would be zero. The candidate solution is to set $\left|\rho_{12}\right|$ to unity and compute the corresponding optimal values of the allocated powers. Substituting $\left|\rho_{12}\right|=1$ in (C.4a) and (C.4b) results in

$$
\begin{aligned}
\sigma_{1}^{2}\left[\left|\mathbf{h}_{1}\right|^{2}-\nu\right] & =-\sigma_{1} \sigma_{2} \Re\left[\rho_{12} \mathbf{h}_{2}^{H} \mathbf{h}_{1}\right], \\
\sigma_{2}^{2}\left[\left|\mathbf{h}_{2}\right|^{2}-\nu\right] & =-\sigma_{1} \sigma_{2} \Re\left[\rho_{12} \mathbf{h}_{2}^{H} \mathbf{h}_{1}\right] .
\end{aligned}
$$

Using (C.8a) and (C.8b), we may get $\frac{\sigma_{1}^{2}-\sigma_{2}^{2}}{\sigma_{1} \sigma_{2}}=\frac{\left|\mathbf{h}_{1}\right|^{2}-\left|\mathbf{h}_{2}\right|^{2}}{\Re\left[\rho_{12} \mathbf{h}_{2}^{H} \mathbf{h}_{1}\right]}$ which can be solved numerically for $\sigma_{1}^{2}$ and $\sigma_{2}^{2}$. 
The $c_{i j}$ expressions in (5.24)-(5.26) are given as:

$$
\begin{aligned}
c_{21}= & \sigma_{1}^{2} \sigma_{2} \sigma_{3}\left[\rho_{31} \rho_{12}\left[\mathbf{h}_{1}^{H} \mathbf{h}_{2} \mathbf{h}_{2}^{H} \mathbf{h}_{3}-\mathbf{h}_{1}^{H} \mathbf{h}_{3}\left|\mathbf{h}_{2}\right|^{2}\right]+\rho_{32}\left[\mathbf{h}_{1}^{H} \mathbf{h}_{3}\left|\mathbf{h}_{2}\right|^{2}-\mathbf{h}_{1}^{H} \mathbf{h}_{2} \mathbf{h}_{2}^{H} \mathbf{h}_{3}\right]\right] \\
& +\sigma_{1}^{2} \sigma_{3}^{2}\left[\mathbf{h}_{3}^{H} \mathbf{h}_{2} \mathbf{h}_{1}^{H} \mathbf{h}_{3}-\mathbf{h}_{1}^{H} \mathbf{h}_{2}\left|\mathbf{h}_{3}\right|^{2}+\left|\rho_{13}\right|^{2}\left[\mathbf{h}_{1}^{H} \mathbf{h}_{2}\left|\mathbf{h}_{3}\right|^{2}-\mathbf{h}_{3}^{H} \mathbf{h}_{2} \mathbf{h}_{1}^{H} \mathbf{h}_{3}\right]\right] \\
& +\sigma_{1} \sigma_{2} \sigma_{3}^{2}\left[\rho_{12}\left[\left|\mathbf{h}_{2}^{H} \mathbf{h}_{3}\right|^{2}-\left|\mathbf{h}_{2}\right|^{2}\left|\mathbf{h}_{3}\right|^{2}\right]+\rho_{32} \rho_{13}\left[\left|\mathbf{h}_{2}\right|^{2}\left|\mathbf{h}_{3}\right|^{2}-\left|\mathbf{h}_{2}^{H} \mathbf{h}_{3}\right|^{2}\right]\right] \\
& -\sigma_{1}^{2} \mathbf{h}_{1}^{H} \mathbf{h}_{2}-\rho_{12} \sigma_{1} \sigma_{2}\left|\mathbf{h}_{2}\right|^{2}-\rho_{13} \sigma_{1} \sigma_{3} \mathbf{h}_{3}^{H} \mathbf{h}_{2},
\end{aligned}
$$

$$
\begin{aligned}
c_{22}= & \sigma_{1}^{2} \sigma_{2} \sigma_{3}\left[\rho_{31} \rho_{12}\left[\mathbf{h}_{2}^{H} \mathbf{h}_{1} \mathbf{h}_{1}^{H} \mathbf{h}_{3}-\mathbf{h}_{2}^{H} \mathbf{h}_{3}\left|\mathbf{h}_{1}\right|^{2}\right]+\rho_{32}\left[\mathbf{h}_{2}^{H} \mathbf{h}_{3}\left|\mathbf{h}_{1}\right|^{2}-\mathbf{h}_{1}^{H} \mathbf{h}_{3} \mathbf{h}_{2}^{H} \mathbf{h}_{1}\right]\right] \\
& +\sigma_{1}^{2} \sigma_{3}^{2}\left[\left|\mathbf{h}_{1}\right|^{2}\left|\mathbf{h}_{3}\right|^{2}-\left|\mathbf{h}_{1}^{H} \mathbf{h}_{3}\right|^{2}+\left|\rho_{13}\right|^{2}\left[\mathbf{h}_{3}^{H} \mathbf{h}_{\mathbf{1}} \mathbf{h}_{1}^{H} \mathbf{h}_{2}-\left|\mathbf{h}_{1}\right|^{2}\left|\mathbf{h}_{3}\right|^{2}\right]\right] \\
& +\sigma_{1} \sigma_{2} \sigma_{3}^{2}\left[\rho_{12}\left[\mathbf{h}_{2}^{H} \mathbf{h}_{1}\left|\mathbf{h}_{3}\right|^{2}-\mathbf{h}_{2}^{H} \mathbf{h}_{3} \mathbf{h}_{3}^{H} \mathbf{h}_{1}\right]+\rho_{32} \rho_{13}\left[\mathbf{h}_{3}^{H} \mathbf{h}_{1} \mathbf{h}_{2}^{H} \mathbf{h}_{3}\right]\right] \\
& +1+\left.\sigma_{1}^{2}\left|\mathbf{h}_{1}\right|^{2}\left|+\rho_{12} \sigma_{1} \sigma_{2} \mathbf{h}_{2}^{H} \mathbf{h}_{\mathbf{1}}+\rho_{31} \sigma_{1} \sigma_{3} \mathbf{h}_{1}^{H} \mathbf{h}_{3}+\rho_{13} \sigma_{1} \sigma_{3} \mathbf{h}_{3}^{H} \mathbf{h}_{1}+\sigma_{3}^{2}\right| \mathbf{h}_{3}\right|^{2} \mid \\
& +\rho_{32} \sigma_{2} \sigma_{3} \mathbf{h}_{2}^{H} \mathbf{h}_{3}
\end{aligned}
$$

$$
\begin{aligned}
c_{23}= & \sigma_{1}^{2} \sigma_{2} \sigma_{3}\left[\rho_{32}\left[\left|\mathbf{h}_{1}^{H} \mathbf{h}_{2}\right|^{2}-\left|\mathbf{h}_{1}\right|^{2}\left|\mathbf{h}_{2}\right|^{2}\right]+\rho_{12} \rho_{31}\left[\left|\mathbf{h}_{1}\right|^{2}\left|\mathbf{h}_{2}\right|^{2}-\left|\mathbf{h}_{1}^{H} \mathbf{h}_{2}\right|^{2}\right]\right] \\
& +\sigma_{1}^{2} \sigma_{3}^{2}\left[\mathbf{h}_{1}^{H} \mathbf{h}_{2} \mathbf{h}_{3}^{H} \mathbf{h}_{1}-\mathbf{h}_{3}^{H} \mathbf{h}_{2}\left|\mathbf{h}_{1}\right|^{2}+\left|\rho_{13}\right|^{2}\left[\mathbf{h}_{3}^{H} \mathbf{h}_{2}\left|\mathbf{h}_{1}\right|^{2}-\mathbf{h}_{1}^{H} \mathbf{h}_{2} \mathbf{h}_{3}^{H} \mathbf{h}_{1}\right]\right] \\
& +\sigma_{1} \sigma_{2} \sigma_{3}^{2}\left[\rho_{12}\left[\mathbf{h}_{3}^{H} \mathbf{h}_{2}\left|\mathbf{h}_{2}\right|^{2}-\mathbf{h}_{2}^{H} \mathbf{h}_{1} \mathbf{h}_{3}^{H} \mathbf{h}_{2}\right]+\rho_{32} \rho_{13}\left[\mathbf{h}_{3}^{H} \mathbf{h}_{2} \mathbf{h}_{2}^{H} \mathbf{h}_{1}-\mathbf{h}_{3}^{H} \mathbf{h}_{1}\left|\mathbf{h}_{2}\right|^{2}\right]\right] \\
& -\rho_{13} \sigma_{1} \sigma_{2} \mathbf{h}_{1}^{H} \mathbf{h}_{2}-\rho_{32} \sigma_{2} \sigma_{3}\left|\mathbf{h}_{2}\right|^{2}-\sigma_{3}^{2} \mathbf{h}_{3}^{H} \mathbf{h}_{2},
\end{aligned}
$$




$$
\begin{aligned}
c_{31}= & \sigma_{1}^{2} \sigma_{2} \sigma_{3}\left[\rho_{13} \rho_{21}\left[\mathbf{h}_{3}^{H} \mathbf{h}_{2} \mathbf{h}_{1}^{H} \mathbf{h}_{3}-\mathbf{h}_{1}^{H} \mathbf{h}_{2}\left|\mathbf{h}_{3}\right|^{2}\right]+\rho_{32}\left[\mathbf{h}_{2}^{H} \mathbf{h}_{3}\left|\mathbf{h}_{1}\right|^{2}-\mathbf{h}_{1}^{H} \mathbf{h}_{3} \mathbf{h}_{2}^{H} \mathbf{h}_{1}\right]\right] \\
& +\sigma_{1}^{2} \sigma_{2}^{2}\left[\mathbf{h}_{1}^{H} \mathbf{h}_{2} \mathbf{h}_{2}^{H} \mathbf{h}_{3}-\mathbf{h}_{1}^{H} \mathbf{h}_{3}\left|\mathbf{h}_{2}\right|^{2}+\left|\rho_{12}\right|^{2}\left[\mathbf{h}_{1}^{H} \mathbf{h}_{3}\left|\mathbf{h}_{2}\right|^{2}-\mathbf{h}_{2}^{H} \mathbf{h}_{3} \mathbf{h}_{1}^{H} \mathbf{h}_{3}\right]\right] \\
& +\sigma_{1} \sigma_{2}^{2} \sigma_{3}\left[\rho_{13}\left[\left|\mathbf{h}_{2}^{H} \mathbf{h}_{3}\right|^{2}-\left|\mathbf{h}_{2}\right|^{2}\left|\mathbf{h}_{3}\right|^{2}\right]+\rho_{12} \rho_{23}\left[\left|\mathbf{h}_{2}\right|^{2}\left|\mathbf{h}_{3}\right|^{2}-\left|\mathbf{h}_{2}^{H} \mathbf{h}_{3}\right|^{2}\right]\right]
\end{aligned}
$$

$$
\begin{aligned}
c_{32}= & \sigma_{1}^{2} \sigma_{2} \sigma_{3}\left[\rho_{13} \rho_{21}\left[\left.\mathbf{h}_{1}\right|^{2}\left|\mathbf{h}_{3}\right|^{2}-\left|\mathbf{h}_{1}^{H} \mathbf{h}_{3}\right|^{2}\right]+\rho_{32}\left[\left|\mathbf{h}_{1}^{H} \mathbf{h}_{3}\right|^{2}-\left|\mathbf{h}_{1}\right|^{2}\left|\mathbf{h}_{3}\right|^{2}\right]\right] \\
& +\sigma_{1}^{2} \sigma_{2}^{2}\left[\mathbf{h}_{2}^{H} \mathbf{h}_{1} \mathbf{h}_{1}^{H} \mathbf{h}_{3}-\mathbf{h}_{2}^{H} \mathbf{h}_{3}\left|\mathbf{h}_{1}\right|^{2}+\left|\rho_{12}\right|^{2}\left[\mathbf{h}_{2}^{H} \mathbf{h}_{3}\left|\mathbf{h}_{1}\right|^{2}-\mathbf{h}_{2}^{H} \mathbf{h}_{1} \mathbf{h}_{1}^{H} \mathbf{h}_{3}\right]\right] \\
& +\sigma_{1} \sigma_{2}^{2} \sigma_{3}\left[\rho_{13}\left[\mathbf{h}_{2}^{H} \mathbf{h}_{1}\left|\mathbf{h}_{3}\right|^{2}-\mathbf{h}_{3}^{H} \mathbf{h}_{1} \mathbf{h}_{2}^{H} \mathbf{h}_{3}\right]+\rho_{12} \rho_{23}\left[\mathbf{h}_{3}^{H} \mathbf{h}_{1} \mathbf{h}_{2}^{H} \mathbf{h}_{3}-\mathbf{h}_{2}^{H} \mathbf{h}_{1}\left|\mathbf{h}_{3}\right|^{2}\right]\right] \\
& -\rho_{12} \sigma_{1} \sigma_{2} \mathbf{h}_{1}^{H} \mathbf{h}_{3}-\sigma_{2}^{2} \mathbf{h}_{2}^{H} \mathbf{h}_{3}-\rho_{12} \sigma_{1} \sigma_{2}\left|\mathbf{h}_{3}\right|^{2}
\end{aligned}
$$

and

$$
\begin{aligned}
c_{33}= & \sigma_{1}^{2} \sigma_{2} \sigma_{3}\left[\rho_{13} \rho_{21}\left[\mathbf{h}_{3}^{H} \mathbf{h}_{1} \mathbf{h}_{1}^{H} \mathbf{h}_{2}-\mathbf{h}_{3}^{H} \mathbf{h}_{2}\left|\mathbf{h}_{1}\right|^{2}\right]+\rho_{32}\left[\mathbf{h}_{3}^{H} \mathbf{h}_{2}\left|\mathbf{h}_{1}\right|^{2}-\mathbf{h}_{3}^{H} \mathbf{h}_{1} \mathbf{h}_{1}^{H} \mathbf{h}_{2}\right]\right] \\
& +\sigma_{1}^{2} \sigma_{2}^{2}\left[\left|\mathbf{h}_{1}\right|^{2}\left|\mathbf{h}_{2}\right|^{2}-\left|\mathbf{h}_{1}^{H} \mathbf{h}_{2}\right|^{2}+\left|\rho_{12}\right|^{2}\left[\left|\mathbf{h}_{1}^{H} \mathbf{h}_{2}\right|^{2}-\left|\mathbf{h}_{1}\right|^{2}\left|\mathbf{h}_{2}\right|^{2}\right]\right] \\
& +\sigma_{1} \sigma_{2}^{2} \sigma_{3}\left[\rho_{13}\left[\mathbf{h}_{3}^{H} \mathbf{h}_{1}\left|\mathbf{h}_{2}\right|^{2}-\mathbf{h}_{2}^{H} \mathbf{h}_{1} \mathbf{h}_{3}^{H} \mathbf{h}_{2}\right]+\rho_{12} \rho_{23}\left[\mathbf{h}_{2}^{H} \mathbf{h}_{1} \mathbf{h}_{3}^{H} \mathbf{h}_{2}-\mathbf{h}_{3}^{H} \mathbf{h}_{1}\left|\mathbf{h}_{2}\right|^{2}\right]\right] \\
& +1+\left.\sigma_{1}^{2}\left|\mathbf{h}_{1}\right|^{2}\left|+\rho_{12} \sigma_{1} \sigma_{2} \mathbf{h}_{2}^{H} \mathbf{h}_{1}+\rho_{13} \sigma_{1} \sigma_{3} \mathbf{h}_{3}^{H} \mathbf{h}_{1}+\rho_{21} \sigma_{1} \sigma_{2} \mathbf{h}_{1}^{H} \mathbf{h}_{2} \sigma_{2}^{2}\right| \mathbf{h}_{2}\right|^{2} \mid \\
& +\rho_{23} \sigma_{2} \sigma_{3} \mathbf{h}_{3}^{H} \mathbf{h}_{2} .
\end{aligned}
$$




\section{Appendix D}

\section{Derivations for Chapter 6}

To express the distribution of the quotient of two generalized- $K$ RVs, we use again the expression of the generalized- $K \mathrm{PDF}$ as a special case of the $H$-function distribution family as in (3.17). Hence, if we denote the quotient of two independent generalized$K$ RVs, $X_{1}$ and $X_{2}$, whose parameters are $m_{m, 1}, m_{s, 1}$, and $\Omega_{0,1} ; m_{m, 2}, m_{s, 2}$, and $\Omega_{0,2}$, respectively, as $\chi=\frac{X_{1}}{X_{2}}$, then, using [Theorem 6.4.3] [50], the PDF of $\chi$ can be expressed as

$$
p(\chi)=\varpi_{1} H_{2,2}^{2,2}\left[\left.\left(\frac{m_{m, 1} m_{s, 1} \Omega_{0,2}}{m_{m, 2} m_{s, 2} \Omega_{0,1}}\right) \chi\right|_{\left(m_{m, 1}-1,1\right),\left(m_{s, 1}-1,1\right)} ^{\left(-m_{m, 2}, 1\right)\left(-m_{s, 2}\right)}\right], \chi>0
$$

where $\varpi_{1}=\frac{m_{m, 1} m_{s, 1} \Omega_{0,2}}{\Gamma\left(m_{m, 1}\right) \Gamma\left(m_{s, 1}\right) \Gamma\left(m_{m, 2}\right) \Gamma\left(m_{s, 2}\right) \Omega_{0,1}}$ which reduces to

$$
p(\chi)=\varpi_{1} G_{2,2}^{2,2}\left[\left.\left(\frac{m_{m, 1} m_{s, 1} \Omega_{0,2}}{m_{m, 2} m_{s, 2} \Omega_{0,1}}\right) \chi\right|_{m_{m, 1}-1, m_{s, 1}-1} ^{-m_{m, 2}-m_{s, 2}}\right], \chi>0 .
$$




\section{Appendix E}

\section{Papers Published, Submitted, and in Preparation}

\section{Chapter 3}

- Saad Al-Ahmadi and Halim Yanikomeroglu, "On the approximation of the generalized- $K$ distribution by a Gamma distribution for modeling composite fading channels," IEEE Trans. Wirel. Commun., vol. 8, no. 2, pp. 586-592, Feb. 2010 .

- Saad Al-Ahmadi and Halim Yanikomeroglu, "On the approximation of the generalized- $K$ PDF by a Gamma PDF using the moment matching method," in Proc. IEEE Wireless Communications and Networking Conference (WCNC), Apr. 2009.

- Saad Al-Ahmadi and Halim Yanikomeroglu, "On the use of high-order moment matching to approximate the generalized- $K$ distribution by a Gamma distribution," Proc. IEEE Global Telecommunications Conference (GLOBECOM), Dec. 2009.

\section{Chapter 4}

- Saad Al-Ahmadi and Halim Yanikomeroglu, "On the approximation of the PDF of the sum of independent generalized- $K$ RVs by another generalized- $K$ PDF 
with applications to distributed antenna systems," appeared in Proc. IEEE Wireless Communications and Networking Conference (WCNC), Apr. 2010.

- Saad Al-Ahmadi and Halim Yanikomeroglu, "On the statistics of the sum of correlated generalized-K RVs," in Proc. IEEE International Conference on Communications (ICC), May 2010.

- Saad Al-Ahmadi and Halim Yanikomeroglu, "On the amount of fading for the sum of correlated generalized-K RVs," submitted to IEEE Transactions on Communications (submission: 18 November 2009, 1st results: 18 February 2010).

\section{Chapter 5}

- Saad Al-Ahmadi and Halim Yanikomeroglu, "On the Beamforming Optimality Range in TIMO Channels with Common and Individual Input Power Constraints," accepted to IEEE Transactions on Communications.

- Saad Al-Ahmadi and Halim Yanikomeroglu, "On the role of the input power constraint in the beamforming optimality range in TIMO channels," 11th Canadian Workshop on Information Theory (CWIT), May 2009.

\section{Chapter 6}

- Saad Al-Ahmadi and Halim Yanikomeroglu, "The ergodic and outage capacities of distributed antenna systems in generalized- $K$ fading channels, accepted to IEEE International Symposium on Personal, Indoor and Mobile Radio Communications (PIMRC), Sept. 2010.

- Saad Al-Ahmadi and Halim Yanikomeroglu, "The information capacity of distributed antenna systems over generalized- $K$ composite fading channels," in preparation for IEEE Journal on Selected Areas in Communications. Special issue on "Distributed Broadband Wireless Communications". 


\section{Bibliography}

[1] D. N. C. Tse and P. Viswanath, Fundamentals of Wireless Communications. Cambridge: Cambridge University Press, 2005.

[2] M. Karakayali, G. Foschini, and R. Valenzuela, "Network coordination for spectrally efficient communications in cellular systems," IEEE Wireless Communications, vol. 13, no. 4, pp. 56-61, Aug. 2006.

[3] O. Simeone, O. Somekh, H. Poor, and S. Shamai, "Local base station cooperation via finite-capacity links for the uplink of linear cellular networks," IEEE Transactions on Information Theory, vol. 55, no. 1, pp. 190-204, Jan. 2009.

[4] J. Zhang, R. Chen, J. Andrews, A. Ghosh, and R. Heath, "Networked MIMO with clustered linear precoding," IEEE Transactions on Wireless Communications, vol. 8, no. 4, pp. 1910-1921, Apr. 2009.

[5] S. A. Ramprashad and G. Caire, "Cellular vs. network MIMO: A comparison including the channel state information overhead," in Proc. of IEEE International Symposium on Personal, Indoor and Mobile Radio Communications (PIMRC), Sept. 2009.

[6] D. A. Palmer and A. J. Motely, "Controlled radio coverage within buildings," British Telecommuinication Technolology Journal, vol. 4, pp. 55-57, Oct. 1986.

[7] A. Saleh, A. Rustako, and R. Roman, "Distributed antennas for indoor radio communications," IEEE Transactions on Communications, vol. 35, no. 12, pp. 1245-1251, Dec. 1987.

[8] A. Salmasi and K. Gilhousen, "On the system design aspects of code division multiple access (CDMA) applied to digital cellular and personal communications networks," in Proc. of IEEE Vehicular Technology Conference (VTC), May 1991.

[9] H. Yanikomeroglu and E. S. Sousa, "CDMA distributed antenna system for indoor wireless communications," in Proc. 2nd International Conference on Universal Personal Communications (ICUPC), Oct. 1993.

[10] H. Xia, A. Herrera, S. Kim, and F. Rico, "A CDMA-distributed antenna system for in-building personal communications services," IEEE Journal on Selected Areas in Communications, vol. 14, no. 4, pp. 644-650, May 1996. 
[11] H. Yanikomeroglu and E. Sousa, "Power control and number of antenna elements in CDMA distributed antenna systems," in Proc. IEEE International Conference on Communications (ICC), June 1998.

[12] A. Obaid and H. Yanikomeroglu, "Reverse-link power control in CDMA distributed antenna systems," in Proc. of IEEE Wireless Communications and Networking Conference (WCNC), 2000.

[13] L. Dai, S. Zhou, and Y. Yao, "Capacity analysis in CDMA distributed antenna systems," IEEE Transactions on Wireless Communications, vol. 4, no. 6, pp. 2613-2620, Nov. 2005.

[14] H. Yanikomeroglu and E. S. Sousa, "CDMA sectorized distributed antenna system," in IEEE 5th International Symposium on Spread Spectrum Techniques and Applications Proceedings, Sep. 1998.

[15] H. Yanikomeroglu and E. Sousa, "SIR-balanced macro power control for the reverse link of CDMA sectorized distributed antenna system," in Proc. of IEEE International Symposium on Personal, Indoor and Mobile Radio Communications (PIMRC), Sep. 1998.

[16] H. Hu, Y. Zhang, and J. Luo, Distributed Antenna Systems: Open Architecture for Future Wireless Communications. CRC: CRC Press, 2007.

[17] P. Marsch and G. Fettweis, "A framework for optimizing the uplink performance of distributed antenna systems under a constrained backhaul," in Proc. IEEE International Conference on Communications (ICC), June 2007.

[18] W. Roh and A. Paulraj, "MIMO channel capacity for the distributed antenna," in Proc. of IEEE Vehicular Technology Conference (VTC), 2002.

[19] —_, "Outage performance of the distributed antenna systems in a composite fading channel," in Proc. of IEEE Vehicular Technology Conference (VTC), 2002.

[20] "D3.4.1: The WINNER II Air Interface: refined spatial-temporal processing solutions," IST-4-027756 WINNER II Deliverable, Oct. 2006.

[21] X.-H. Yu, G. Chen, M. Chen, and X. Gao, "The FuTURE Project in China," IEEE Communications Magazine, vol. 43, no. 1, pp. 70-75, Jan. 2005.

[22] A. Cooper, "Fibre/radio for the provision of cordless/mobile telephony services in the access network," Electronics Letters, vol. 40, no. 3, pp. 599-606, Aug. 1991.

[23] T. S. Chu and M. Gans, "Fiber optic microcellular radio," IEEE Transactions on Vehicular Technology, vol. 40, no. 3, pp. 599-606, Aug. 1991. 
[24] M. Borella, J. Jue, D. Banerjee, B. Ramamurthy, and B. Mukherjee, "Optical components for WDM lightwave networks," Proceedings of the IEEE, vol. 85, no. 8, pp. 1274-1307, Aug. 1997.

[25] Y. Chigusa, Y. Yamamoto, T. Yokokawa, T. Sasaki, T. Taru, M. Hirano, M. Kakui, M. Onishi, and E. Sasaoka, "Low-loss pure-silica-core fibers and their possible impact on transmission systems," Journal of Lightwave Technology, vol. 23, no. 11, pp. 3541-3550, Nov. 2005.

[26] H. Al-Raweshidy and S. Komaki, Radio over Fiber Technologies for Mobile Communications Networks. Norwood: Artech House, 2002.

[27] W. Braun and U. Dersch, "A physical mobile radio channel model," IEEE Transactions on Vehicular Technology, vol. 40, no. 2, pp. 472-482, May 1991.

[28] W. C. J. (ed), Microwave Mobile Communications. New York: John Wiley and Sons, 1974.

[29] A. Papoulis, Probability, Random Variables, and Stochastic Processes. New York: McGraw-Hill, 1984.

[30] S. O. Rice, "Mathematical analysis of random noise," Bell System Technical Journal, vol. 23, no. 1, pp. 282-332, 1944.

[31] A. Annamalai, C. Tellambura, and V. Bhargava, "Simple and accurate methods for outage analysis in cellular mobile radio systems-a unified approach," IEEE Transactions on Communications, vol. 49, no. 2, pp. 303-316, Feb. 2001.

[32] M. Nakagami, "The $m$-distribution: a general formula of intensity distribution of rapid fading," Statistical Methods in Radio Propagation, 1960.

[33] I. S. Gradshteyn and I. M. Ryzhik, Tables of Integrals, Series, and Products. Academic Press, 2007.

[34] N. Shepherd, "Radio wave loss deviation and shadow loss at $900 \mathrm{mhz}$," IEEE Transactions on Vehicular Technology, vol. 26, no. 4, pp. 309-313, Nov. 1977.

[35] J. Cheng, C. Tellambura, and N. Beaulieu, "Performance of digital linear modulations on Weibull slow-fading channels," IEEE Transactions on Communications, vol. 52, no. 8, pp. 1265-1268, Aug. 2004.

[36] A. Abdi, "On the utility of Laguerre series for the envelope pdf in multipath fading channels," IEEE Transactions on Information Theory, vol. 55, no. 12, pp. 5652-5660, Dec. 2009.

[37] M. Yacoub, "The $\kappa-\mu$ distribution and the $\eta-\mu$ distribution," IEEE Antennas and Propagation Magazine, vol. 49, no. 1, pp. 68-81, Feb. 2007. 
[38] A. Coulson, A. Williamson, and R. Vaughan, "A statistical basis for lognormal shadowing effects in multipath fading channels," IEEE Transactions on Communications, vol. 46, no. 4, pp. 494-502, Apr. 1998.

[39] G. L. Stuber, Principles of Mobile Communication. Norwell, MA: Kluwer, 2001.

[40] A. Abdi and M. Kaveh, "K distribution: an appropriate substitute for rayleighlognormal distribution in fading-shadowing wireless channels," Electronics Letters, vol. 34, no. 9, pp. 851-852, Apr. 1998.

[41] —-, "On the utility of gamma PDF in modeling shadow fading (slow fading)," in Proc. of IEEE Vehicular Technology Conference (VTC), Jul. 1999.

[42] J. Salo, L. Vuokko, H. M. El-Sallabi, and P. Vainikainen, "An additive model as a physical basis for shadow fading," IEEE Transactions on Vehicular Technology, vol. 56, no. 1, pp. 13-26, Jan. 2007.

[43] C. Tellambura and V. Bhargava, "Outage probability analysis for cellular mobile radio systems subject to Nakagami fading and shadowing," IEICE Transactions on Communications, vol. E78-B, no. 10, pp. 1416-1423, Oct. 1995.

[44] D. Lewinski, "Nonstationary probabilistic target and clutter scattering models," IEEE Transactions on Antennas and Propagation, vol. 31, no. 3, pp. 490-498, May 1983.

[45] P. M. Shankar, "Error rates in generalized shadowed fading channels," Wireless Personal Communications, vol. 28, no. 4, pp. 233-238, Feb. 2004.

[46] E. Jakeman and P. Pusey, "A model for non-rayleigh sea echo," IEEE Transactions on Antennas and Propagation, vol. 24, no. 6, pp. 806-814, Nov. 1976.

[47] R. Barakat, "Weak-scatterer generalization of the k-density function with application to laser scattering in atmospheric turbulence," Journal of the Optical Society of America, vol. 3, no. 4, pp. 401-409, Apr. 1986.

[48] E. Bayaki, R. Schober, and R. Mallik, "Performance analysis of MIMO freespace optical systems in gamma-gamma fading," IEEE Transactions on Communications, vol. 57, no. 11, pp. 3415-3424, Nov. 2009.

[49] P. Bithas, N. Sagias, P. Mathiopoulos, G. Karagiannidis, and A. A. Rontogiannis, "On the performance analysis of digital communications over generalized-K fading channels," IEEE Communications Letters, vol. 10, no. 5, pp. 353-355, May 2006.

[50] M. D. Springer, The Algebra of Random Variables. New York: John Wiley and Sons, 1979. 
[51] V. S. Adamchik and O. I. Marichev, "The algorithm for calculating integrals of hypergeometric type functions and its realization in REDUCE system," in Proc. of the International Symposium on Symbolic and Algebraic Computation (ISSAC), 1990.

[52] P. Bithas, P. Mathiopoulos, and S. Kotsopoulos, "Diversity reception over generalized-K KG fading channels," IEEE Transactions on Wireless Communications, vol. 6, no. 12, pp. 4238-4243, Dec. 2007.

[53] I. Kostic, "Analytical approach to performance analysis for channel subject to shadowing and fading," IEE Proceedings-Communications, vol. 152, no. 6, pp. 821-827, Dec. 2005.

[54] N. Cressie, A. S. Davis, J. L. Folks, and G. Policello, "The moment-generating function and negative integer moments," The American Statistician, vol. 35, no. 3, pp. 148-150, Aug. 1981.

[55] J. Santos Filho, M. Yacoub, and P. Cardieri, "Highly accurate range-adaptive lognormal approximation to lognormal sum distributions," Electronics Letters, vol. 42, no. 6, pp. 361-363, Mar. 2006.

[56] A. Charash, A Study of Multipath Reception with Unknown Delays. University of California, Berkeley: UC, 1974.

[57] B. Lindsay and K. Roeder, "Moment-based oscillations properties of mixture models," Annals of Statistics, vol. 25, no. 1, pp. 378-386, Feb. 1997.

[58] A. Abdi, W. Lau, M.-S. Alouini, and M. Kaveh, "A new simple model for land mobile satellite channels: first-and second-order statistics," IEEE Transactions on Wireless Communications, vol. 2, no. 3, pp. 519-528, May 2003.

[59] N. Beaulieu and Q. Xie, "An optimal lognormal approximation to lognormal sum distributions," IEEE Transactions on Vehicular Technology, vol. 53, no. 2, pp. 479-489, Mar. 2004.

[60] T. M. Cover and J. A. Thomas, Elements of Information Theory. New York: John Wiley and Sons, 2006.

[61] S. Al-Ahmadi and H. Yanikomeroglu, "On the approximation of the generalized$\mathrm{K}$ distribution by a gamma distribution for modeling composite fading channels," IEEE Transactions on Wireless Communications, vol. 9, no. 2, pp. 706713, Feb. 2010.

[62] B. Armstrong and H. Griffiths, "CFAR detection of fluctuating targets in spatially correlated K-distributed clutter," IEE Proceedings Radar and Signal Processing, vol. 138, no. 2, pp. 139-152, Apr. 1991. 
[63] C. Zhu, J. Mietzner, and R. Schober, "On the performance of non-coherent transmission schemes with equal-gain combining in generalized-K fading," IEEE Transactions on Wireless Communications, vol. 9, no. 4, pp. 1337-1349, Apr. 2010.

[64] M. K. Simon and M. S. Alouini, Digital Communication Over Fading Channels. Hoboken, NJ: John Wiley and Sons, 2005.

[65] P. Bithas, N. Sagias, and P. Mathiopoulos, "The bivariate generalized-K KG distribution and its application to diversity receivers," IEEE Transactions on Communications, vol. 57, no. 9, pp. 2655-2662, Sep. 2009.

[66] P. G. Moschopoulos, "The distribution of the sum of independent gamma random variables," Annals of the Institute of Statistical Mathematics, vol. 37, no. 1, pp. 541-544, Dec. 1985.

[67] G. Karagiannidis, N. Sagias, and T. Tsiftsis, "Closed-form statistics for the sum of squared Nakagami-m variates and its applications," IEEE Transactions on Communications, vol. 54, no. 8, pp. 1353-1359, Aug. 2006.

[68] Z. Wang and G. Giannakis, "A simple and general parameterization quantifying performance in fading channels," IEEE Transactions on Communications, vol. 51, no. 8, pp. 1389-1398, Aug. 2003.

[69] H. Nie and S. Chen, "Lognormal sum approximation with type IV Pearson distribution," IEEE Communications Letters, vol. 11, no. 10, pp. 790-792, Oct. 2007.

[70] N. D. Chatzidiamantis, G. K. Karagiannidis, and D. S. Michalopoulos, "On the distribution of the sum of gamma-gamma variates and application in MIMO optical wireless systems," in Proc. IEEE Global Telecommunications Conference (GLOBECOM), Dec. 2009.

[71] P. M. Shankar, "Performance analysis of diversity combining algorithms in shadowed fading channels," Wireless Personal Communications, vol. 37, no. 2, pp. 61-72, Apr. 2006.

[72] M.-S. Alouini, A. Abdi, and M. Kaveh, "Sum of gamma variates and performance of wireless communication systems over Nakagami-fading channels," IEEE Transactions on Vehicular Technology, vol. 50, no. 6, pp. 1471-1480, Nov. 2001.

[73] K. Butterworth, K. Sowerby, and A. Williamson, "Correlated shadowing in an in-building propagation environment," Electronics Letters, vol. 33, no. 5, pp. 420-422, Feb. 1997.

[74] E. Perahia, D. Cox, and S. Ho, "Shadow fading cross correlation between basestations," in Proc. of IEEE Vehicular Technology Conference (VTC), 2001. 
[75] J. E. Prussing, "The principal minor test for semidefinite matrices," Journal of Guidance, vol. 9, no. 1, pp. 121-122, Jan.-Feb. 1986.

[76] C. D. Meyer, Matrix Analysis and Applied Linear Algebra. Philadelphia, PA: SIAM, 2000.

[77] F. Graziosi and F. Santucci, "A general correlation model for shadow fading in mobile radio systems," IEEE Communications Letters, vol. 6, no. 3, pp. 102-104, Mar. 2002.

[78] Z. Wang, E. Tameh, and A. Nix, "Joint shadowing process in urban peer-to-peer radio channels," IEEE Transactions on Vehicular Technology, vol. 57, no. 1, pp. 52-64, Jan. 2008.

[79] Y. Li and S. Kishore, "Diversity factor-based capacity asymptotic approximations of MRC reception in rayleigh fading channels," IEEE Transactions on Communications, vol. 56, no. 6, pp. 858-861, June 2008.

[80] G. J. Foschini and M. J. Gans, "On limits of wireless communications in a fading environment when using multiple antennas," Wireless Personal Communications, vol. 6, pp. 311-335, 1998.

[81] I. E. Telatar, "Capacity of multi-antenna gaussian channel," European Transactions on Telecommunications, vol. 10, pp. 585-595, Nov. 1999.

[82] R. G. Gallager, Information Theory and Reliable Communication. New York: John Wiley and Sons, 1968.

[83] D.-S. Shiu, G. Foschini, M. Gans, and J. Kahn, "Fading correlation and its effect on the capacity of multi-element antenna systems," IEEE Transactions on Communications, vol. 48, no. 3, pp. 502-513, Mar. 2000.

[84] C.-N. Chuah, D. Tse, J. Kahn, and R. Valenzuela, "Capacity scaling in MIMO wireless systems under correlated fading," IEEE Transactions on Information Theory, vol. 48, no. 3, pp. 637-650, Mar. 2002.

[85] A. Goldsmith, S. Jafar, N. Jindal, and S. Vishwanath, "Capacity limits of MIMO channels," IEEE Journal on Selected Areas in Communications, vol. 21, no. 5, pp. 684-702, June 2003.

[86] S. Jafar and A. Goldsmith, "Transmitter optimization and optimality of beamforming for multiple antenna systems," IEEE Transactions on Wireless Communications, vol. 3, no. 4, pp. 1165-1175, Jul. 2004.

[87] E. Jorswieck and H. Boche, "Channel capacity and capacity-range of beamforming in mimo wireless systems under correlated fading with covariance feedback," IEEE Transactions on Wireless Communications, vol. 3, no. 5, pp. 1543-1553, Sep. 2004. 
[88] A. Tulino, A. Lozano, and S. Verdu, "Capacity-achieving input covariance for single-user multi-antenna channels," IEEE Transactions on Wireless Communications, vol. 5, no. 3, pp. 662-671, Mar. 2006.

[89] X. Zheng, Y. Xie, J. Li, and P. Stoica, "MIMO transmit beamforming under uniform elemental power constraint," IEEE Transactions on Signal Processing, vol. 55 , no. 11 , pp. 5395-5406, Nov. 2007.

[90] F. Neeser and J. Massey, "Proper complex random processes with applications to information theory," IEEE Transactions on Information Theory, vol. 39, no. 4, pp. 1293-1302, Jul. 1993.

[91] J. Andersen, "Array gain and capacity for known random channels with multiple element arrays at both ends," IEEE Journal on Selected Areas in Communications, vol. 18, no. 11, pp. 2172-2178, Nov. 2000.

[92] V. Veeravalli, Y. Liang, and A. Sayeed, "Correlated MIMO wireless channels: capacity, optimal signaling, and asymptotics," IEEE Transactions on Information Theory, vol. 51, no. 6, pp. 2058-2072, June 2005.

[93] M. Vu and A. Paulraj, "On the capacity of MIMO wireless channels with dynamic CSIT," IEEE Journal on Selected Areas in Communications, vol. 25, no. 7 , pp. 1269-1283, Sep. 2007.

[94] M. Ivrlac, W. Utschick, and J. Nossek, "Fading correlations in wireless MIMO communication systems," IEEE Journal on Selected Areas in Communications, vol. 21, no. 5, pp. 819-828, June 2003.

[95] M. Medard, "Processing of wireless signals to preserve wireline network resources," in Proc. of IEEE Wireless Communications and Networking Conference $(W C N C)$, Sep. 1999.

[96] W. Roh and A. Paulraj, "Performance of the distributed antenna systems in a multi-cell environment," in Proc. of IEEE Vehicular Technology Conference (VTC), Apr. 2003.

[97] W. Choi and J. G. Andrews, "Downlink performance and capacity of distributed antenna systems in a multicell environment," IEEE Transactions on Wireless Communications, vol. 6, no. 1, pp. 69-73, Jan. 2007.

[98] J. Park, E. Song, and W. Sung, "Capacity analysis for distributed antenna systems using cooperative transmission schemes in fading channels," IEEE Transactions on Wireless Communications, vol. 8, no. 2, pp. 586-592, Feb. 2009.

[99] O. Oyman, R. Nabar, H. Bolcskei, and A. Paulraj, "Characterizing the statistical properties of mutual information in MIMO channels," IEEE Transactions on Signal Processing, vol. 51, no. 11, pp. 2784-2795, Nov. 2003. 
[100] M. Sharif and B. Hassibi, "On the capacity of MIMO broadcast channels with partial side information," IEEE Transactions on Information Theory, vol. 51, no. 2, pp. 506-522, Feb. 2005.

[101] R. P. B.G. Lindsay and P. Basak, "Moment-based approximations of distributions using mixtures: Theory and applications," Annals of the Institute of Statistical Mathematics, vol. 52, no. 2, pp. 215-230, Nov. 2000.

[102] S. Atapattu, C. Tellambura, and H. Jiang, "Representation of composite fading and shadowing distributions by using mixtures of Gamma distributions," in Proc. of IEEE Wireless Communications and Networking Conference (WCNC), Apr. 2010.

[103] A. M. Mathai and R. K. Saxena, The H-function with Applications in Statistics. New York: Wiley Halsted, 1978.

[104] M. Kendall, Multivariate Analysis. London: Griffin, 1975.

[105] B. D. Finniti, "A proposito di correlazione," Storia ed Economia, vol. 3, pp. 4157, 1937. An English version, translated by L. Barone and P. Laurence in 2006, appears as "About correlation," in Insurance, Mathematics and Economics, 2007. 